\title{
HEAT KERNEL ESTIMATES FOR SUBORDINATE MARKOV PROCESSES AND THEIR APPLICATIONS
}

\author{
SOOBIN CHO, PANKI KIM， RENMING SONG AND ZORAN VONDRAČEK
}

\begin{abstract}
In this paper, we establish sharp two-sided estimates for transition densities of a large class of subordinate Markov processes. As applications, we show that the parabolic Harnack inequality and Hölder regularity hold for parabolic functions of such processes, and derive sharp two-sided Green function estimates.
\end{abstract}

\section{AMS 2020 Mathematics Subject Classification: Primary 60J35, 60J50, 60J76}

Keywords and phrases: Heat kernel, transition density, subordinator, Markov process, subordinate Markov process, parabolic Harnack inequality, Hölder regularity, Green function, spectral fractional Laplacian

\section{INTRODUCTION}

Transition densities of Markov processes are of central importance in both probability and analysis. The transition density $p(t, x, y)$ of a Markov process $X$ with generator $L$ is the fundamental solution of the equation $\partial_{t} u=L u$. Hence the transition density $p(t, x, y)$ is also known as the heat kernel of $L$. The heat kernel is rarely known explicitly. Due to the importance of heat kernels, there is a huge amount of literature devoted to estimates of heat kernels.

The purpose of this paper is to study heat kernel estimates for subordinate Markov processes. The main motivation comes from [42, where it was established that the jump kernels of subordinate killed Lévy processes have an unusual form not observed before. It is therefore plausible that the heat kernels of those processes will have some new features. It turns out that this is indeed the case. To illustrate the new features, we explain below the motivating and also the simplest example covered by our results.

Let $D \subset \mathbb{R}^{d}, d \geq 1$, be a bounded $C^{1,1}$ open set. For $x \in D$, let $\delta_{D}(x)$ denote the distance between $x$ and $D^{c}$. Let $Y$ be an isotropic $\alpha$-stable process in $\mathbb{R}^{d}, \alpha \in(0,2]$ and let $Y^{D}$ denote the part process of $Y$ killed upon exiting $D$. When $\alpha=2$, we further assume that $D$ is connected. Sharp two-sided estimates of the heat kernel $p_{D}(t, x, y)$ of $Y^{D}$ were obtained in [27, 53] (for $\left.\alpha=2\right)$ and [9] (for $\alpha<2$ ): there exist positive constants $c_{i}, i=1, \ldots, 8$, such that following estimates hold true. For $(t, x, y) \in(0,1] \times D \times D$,

$$
c_{1} h(t, x, y)\left(t^{-d / \alpha} \wedge \frac{t}{|x-y|^{d+\alpha}}\right) \leq p_{D}(t, x, y) \leq c_{2} h(t, x, y)\left(t^{-d / \alpha} \wedge \frac{t}{|x-y|^{d+\alpha}}\right), \quad \text { for } \alpha<2,
$$

and

$$
c_{3} h(t, x, y) t^{-d / 2} e^{-c_{4}|x-y|^{2} / t} \leq p_{D}(t, x, y) \leq c_{5} h(t, x, y) t^{-d / 2} e^{-c_{6}|x-y|^{2} / t}, \quad \text { for } \alpha=2,
$$

where the boundary function $h(t, x, y)$ is given by

$$
h(t, x, y)=\left(1 \wedge \frac{\delta_{D}(x)^{\alpha}}{t}\right)^{1 / 2}\left(1 \wedge \frac{\delta_{D}(y)^{\alpha}}{t}\right)^{1 / 2}=\left(1 \wedge \frac{\delta_{D}(x)}{t^{1 / \alpha}}\right)^{\alpha / 2}\left(1 \wedge \frac{\delta_{D}(y)}{t^{1 / \alpha}}\right)^{\alpha / 2} .
$$

For $(t, x, y) \in[1, \infty) \times D \times D$,

$$
c_{7} e^{-\lambda_{1} t} \delta_{D}(x)^{\alpha / 2} \delta_{D}(y)^{\alpha / 2} \leq p_{D}(t, x, y) \leq c_{8} e^{-\lambda_{1} t} \delta_{D}(x)^{\alpha / 2} \delta_{D}(y)^{\alpha / 2},
$$

where $\lambda_{1}$ is the smallest eigenvalue of the Dirichlet (fractional) Laplacian $\left.(-\Delta)^{\alpha / 2}\right|_{D}$.

Let $S=\left(S_{t}\right)_{t \geq 0}$ be a $\beta$-stable subordinator, $\beta \in(0,1)$, independent of $Y^{D}$, and let $X=\left(X_{t}\right)_{t \geq 0}$ be the subordinate process: $X_{t}:=Y_{S_{t}}^{D}$. The generator of $X$ is equal to (the negative of) $\left(\left.(-\Delta)^{\alpha / 2}\right|_{D}\right)^{\beta}-$

The research of Soobin Cho is supported by the POSCO Science Fellowship of POSCO TJ Park Foundation.

This research is supported by the National Research Foundation of Korea(NRF) grant funded by the Korea government(MSIP) (No. NRF-2021R1A4A1027378).

Research supported in part by a grant from the Simons Foundation (\#429343, Renming Song).

Research supported in part by the Croatian Science Foundation under the project 4197. (Zoran Vondraček). 
the fractional power of the Dirichlet fractional Laplacian. In particular, when $\alpha=2,\left(-\left.\Delta\right|_{D}\right)^{\beta}$ is called a spectral fractional Laplacian in the PDE literature, see, for instance, [5] and the references therein. In this respect, the process $X$ bears some similarity with the isotropic $\alpha \beta$-stable process. The heat kernel $q(t, x, y)$ of the subordinate process $X$ is given by

$$
q(t, x, y)=\int_{0}^{\infty} p_{D}(s, x, y) \mathbb{P}\left(S_{t} \in d s\right), \quad t>0, x, y \in D .
$$

Note that the distribution of $S_{t}$ is not explicitly known, making the above integration rather delicate. To handle this integral, we establish some estimates on the distribution of $S_{t}$ (in fact, for much more general subordinators than the stable ones). Using these, we can obtain sharp two-sided estimates of $q(t, x, y)$. To present those estimates, we first introduce some notation. Denote $a \wedge b:=\min \{a, b\}$ and $a \vee b:=\max \{a, b\}$. The notation $f(s) \simeq g(s)$ means that there exist comparison constants $c_{1}, c_{2}>0$ such that $c_{1} g(s) \leq f(s) \leq c_{2} g(s)$ for specified range of the variable $s$. For $x, y \in D$, let

$$
\delta_{\vee}(x, y)=\delta_{D}(x) \vee \delta_{D}(y), \quad \delta_{\wedge}(x, y)=\delta_{D}(x) \wedge \delta_{D}(y) .
$$

Our main results, specialized to the present situation, are summarized below.

Theorem 1.1. (1) For all $(t, x, y) \in(0,1] \times D \times D$,

$$
\begin{aligned}
q(t, x, y) & \simeq\left(1 \wedge \frac{\delta_{D}(x)}{t^{1 /(\alpha \beta)}}\right)^{\alpha / 2}\left(1 \wedge \frac{\delta_{D}(y)}{t^{1 /(\alpha \beta)}}\right)^{\alpha / 2} B^{\alpha, \beta}(t, x, y)\left(t^{-d /(\alpha \beta)} \wedge \frac{t}{|x-y|^{d+\alpha \beta}}\right) \\
& \simeq\left(1 \wedge \frac{\delta_{D}(x)}{t^{1 /(\alpha \beta)}}\right)^{\alpha / 2}\left(1 \wedge \frac{\delta_{D}(y)}{t^{1 /(\alpha \beta)}}\right)^{\alpha / 2}\left(t^{-d /(\alpha \beta)} \wedge \frac{t B^{\alpha, \beta}(t, x, y)}{|x-y|^{d+\alpha \beta}}\right),
\end{aligned}
$$

where

and for $\alpha<2$,

$$
B^{2, \beta}(t, x, y):=\left(1 \wedge \frac{\delta_{D}(x) \vee t^{1 /(2 \beta)}}{|x-y|}\right)\left(1 \wedge \frac{\delta_{D}(y) \vee t^{1 /(2 \beta)}}{|x-y|}\right)
$$

$$
B^{\alpha, \beta}(t, x, y):= \begin{cases}\left(1 \wedge \frac{\delta_{\wedge}(x, y) \vee t^{1 /(\alpha \beta)}}{|x-y|}\right)^{\alpha-\alpha \beta}, & \text { if } \beta>\frac{1}{2}, \\ \left(1 \wedge \frac{\delta_{\wedge}(x, y) \vee t^{1 /(\alpha \beta)}}{|x-y|}\right)^{\alpha / 2}\left(1 \wedge \frac{\delta_{\vee}(x, y) \vee t^{1 /(\alpha \beta)}}{|x-y|}\right)^{\alpha / 2-\alpha \beta}, & \text { if } \beta<\frac{1}{2}, \\ \left(1 \wedge \frac{\delta_{\wedge}(x, y) \vee t^{1 /(\alpha \beta)}}{|x-y|}\right)^{\alpha / 2} \log \left(e+\frac{\left(\delta_{\vee}(x, y) \vee t^{1 /(\alpha \beta)}\right) \wedge|x-y|}{\left(\delta_{\wedge}(x, y) \vee t^{1 /(\alpha \beta)}\right) \wedge|x-y|}\right), & \text { if } \beta=\frac{1}{2} .\end{cases}
$$

(2) For all $(t, x, y) \in[1, \infty) \times D \times D$,

$$
q(t, x, y) \simeq e^{-t \lambda_{1}^{\beta}} \delta_{D}(x)^{\alpha / 2} \delta_{D}(y)^{\alpha / 2},
$$

where $\lambda_{1}$ is the smallest eigenvalue of the Dirichlet (fractional) Laplacian $\left.(-\Delta)^{\alpha / 2}\right|_{D}$.

Remark 1.2. (1) From the forms of the heat kernel estimates (1.1)-(1.2) in Theorem[1.1(1), one can easily see the following: (1-a) For $x, y$ away from the boundary (in the sense that $\delta_{\wedge}(x, y) \geq|x-y| \vee t^{1 /(\alpha \beta)}$ ), and for all $\beta \in(0,1)$, it holds that

$$
q(t, x, y) \simeq t^{-d /(\alpha \beta)} \wedge \frac{t}{|x-y|^{d+\alpha \beta}} .
$$

(1-b) Dividing (1.1) by $t$ and letting $t \rightarrow 0$, we can deduce that the jump kernel is comparable with

$$
\frac{B^{\alpha, \beta}(0, x, y)}{|x-y|^{d+\alpha \beta}} .
$$

This indicates that the term $B^{\alpha, \beta}(t, x, y)$ in (1.1) comes from the boundary decay of the jump kernel.

(2) Using the identity

$$
\left(1 \wedge \frac{r}{a}\right)\left(1 \wedge \frac{r \vee a}{c}\right)=\left(1 \wedge \frac{r}{a \vee c}\right), \quad a, c, r>0,
$$

we see from Theorem 1.1(1) that, when $\alpha=2$,

$$
q(t, x, y) \simeq\left(1 \wedge \frac{\delta_{D}(x)}{|x-y| \vee t^{1 / \beta}}\right)\left(1 \wedge \frac{\delta_{D}(y)}{|x-y| \vee t^{1 / \beta}}\right)\left(t^{-d /(2 \beta)} \wedge \frac{t}{|x-y|^{d+2 \beta}}\right) .
$$


Recall that the two-sided estimates of the form (1.3) are valid for the heat kernel of the isotropic $\alpha \beta$-stable process in the whole space. The novelty of the estimates for $q(t, x, y)$ is in the boundary term, which is quite unusual and involves interplays among $\delta_{\vee}(x, y), \delta_{\wedge}(x, y)$ and time $t$ itself. In this respect, the form of the boundary term is very different from the boundary function $h(t, x, y)$ for the underlying process $Y^{D}$.

All the estimates in Theorem 1.1 are consequences of Theorems 4.2, 4.7 and Lemma 7.1, see Example 7.2. Integrating the heat kernel estimates, we can obtain sharp two-sided estimates on the Green function of $X$, see Theorem 5.8 and Example 7.2

In this paper, we obtain sharp two-sided heat kernel estimates for subordinate Markov processes in a setting which is more general, in several directions, than that of the example above. We allow (i) quite general subordinators, (ii) Markov processes with state space $D$ that is either a bounded or an unbounded subset of a locally compact separable metric space, and (iii) very general form of two-sided estimates of the heat kernel $p_{D}(t, x, y)$ of the underlying process. In the remaining part of the introduction, we describe some of our assumptions and results, and lay out the structure of the paper.

In Section 2, we first introduce the main assumption on the subordinator $S=\left(S_{t}\right)_{t \geq 0}$. Let $\nu$ denote its Lévy measure and $w(t):=\nu(t, \infty)$. We assume that there exist constants $R_{1} \in(0, \infty], c_{1}, c_{2}>0$ and $\beta_{2} \geq \beta_{1}>0$ such that

$$
c_{1}\left(\frac{R}{r}\right)^{\beta_{1}} \leq \frac{w(r)}{w(R)} \leq c_{2}\left(\frac{R}{r}\right)^{\beta_{2}} \text { for all } 0<r \leq R<R_{1} .
$$

This assumption is quite weak - it implies that the Laplace exponent $\phi$ of the subordinator $S$ satisfies weak scaling conditions near infinity with lower index $\beta_{1}$ and upper index $\beta_{2} \wedge 1$. Note that $\beta_{2}>1$ is allowed. Building upon the results from [22, 23, we show several auxiliary results leading to the important estimate (2.24) saying that $\mathbb{P}\left(S_{t} \geq s\right) \simeq t w(s)$ for $2 \phi^{-1}(1 / t)^{-1}<s<R_{1} / 2$.

Sections 3 , are central to the paper. We start with the setup in Section 3 , the underlying space is a locally compact separable metric space $E$ with a Radon measure $m$ having full support and satisfying volume doubling conditions. The state space is a proper open subset $D$ of $E$, bounded or unbounded, and $Y^{D}$ is a Hunt process living on $D$. We assume that $Y^{D}$ admits a transition density $p_{D}(t, x, y)$. The main assumption on the transition density is given in Definition 3.6. Depending on whether $D$ is bounded or unbounded, the assumptions are somewhat different. Roughly speaking, at least for small times, $p_{D}(t, x, y)$ is comparable to the product of two parts which we may call the boundary part and the interior part. The latter is specified in terms of the volume and two functions $-\Psi \geq \Phi-$ both enjoying the scaling property, and also includes a Gaussian part. We note that although in most examples it holds that $\Psi \simeq \Phi$, allowing for different functions enlarges the scope of examples. The boundary part is described through a boundary function $h(t, x, y)$ which is not specified but is required to satisfy certain assumptions - see Definition 3.3. To justify our assumptions on the heat kernel $p_{D}(t, x, y)$, we provide a number of examples from the literature satisfying those assumptions.

The main object of our study is the subordinate process $X_{t}:=Y_{S_{t}}^{D}$. In Subsection 4.1, see Theorem 4.1. we first establish sharp two-sided estimates of the jump kernel $J(x, y)$ of $X$, thus generalizing [42, Theorem 8.4]. Subsection 4.2 contains sharp two-sided estimates of the heat kernel $q(t, x, y)$ of $X$ which are the main results of the paper. The case of a bounded set $D$ and small time is given in Theorem 4.2 , and the case of unbounded $D$ and all time in Theorem 4.3. The near-diagonal estimates have a rather simple form, but the off-diagonal estimates are quite involved, containing four terms which cannot be compared under general assumptions. The form of the estimates is somewhat simplified in case when the upper scaling index $\beta_{2}<1$ and $\Psi \simeq \Phi$, see Corollary 4.4. Finally, Theorem 4.7 provides the large time estimates in case of bounded $D$.

In Section 5 we apply our sharp two-sided heat kernel estimates to derive sharp two-sided estimates of the Green function $G(x, y)$ of $X$. The most general form of the estimates is given in Proposition 5.3. These can be simplified under additional assumptions on the boundary function $h(t, x, y)$. We obtain several forms of the estimates depending on the relationship between the parameters in the volume doubling condition, the scaling indices of the functions $w$ and $\Phi$, and the parameters coming from $h$. The main results of this section are Theorems 5.8, 5.10 and 5.11

In Section 6 we show that parabolic functions with respect to $X$ satisfy Hölder regularity and the parabolic Harnack inequality. By using the rough upper estimates and the interior estimates for the heat kernel from Proposition 4.5 and Corollary 4.6 together with the jump kernel estimates from Theorem 
4.1. we establish that the process $X$ satisfies all the assumptions from [16, 19] used in the proofs of those results.

Finally, in Section 7, we provide a concrete and explicit example which includes the motivating example from the beginning of this introduction, and, with help of Lemma 7.1, derive the heat kernel estimates using the general Theorems 4.2, 4.3 and 4.7. We also derive the jump kernel estimates and Green function estimates using Theorem 4.1, and Theorems 5.8, 5.10, 5.11 respectively. As an application of these estimates, combined with the main results of [44], we completely determine the region of the parameters where the boundary Harnack principle holds for the process $X_{t}=Y_{S_{t}}^{D}$, where $D$ is the upper half-space, $Y^{D}$ is the process in Example 3.9 (b-4) and $S_{t}$ is an independent $\beta$-stable subordinator, $\beta \in(0,1)$. At the end we provide an interesting example in which the upper scaling index $\beta_{2}>1$ and the two scaling function $\Phi$ and $\Psi$ are different.

Notations: Values of lower case letters with subscripts $c_{i}, i=0,1,2, \ldots$ are fixed in each statement and proof, and the labeling of these constants starts anew in each proof. Recall that $a \wedge b:=\min \{a, b\}$, $a \vee b:=\max \{a, b\}$. We use two notations for comparison of functions. First, the notation $f(x) \simeq g(x)$ means that there exist constants $c_{1}, c_{2}>0$ such that $c_{1} g(x) \leq f(x) \leq c_{2} g(x)$ for specified range of $x$. On the other hand, the notation $f(x) \asymp g_{1}(x)+g_{2}(x) h(c x)$ means that there exist constants $c_{3}, c_{4}, c_{5}, c_{6}>0$ such that $c_{3}\left(g_{1}(x)+g_{2}(x) h\left(c_{4} x\right)\right) \leq f(x) \leq c_{5}\left(g_{1}(x)+g_{2}(x) h\left(c_{6} x\right)\right)$ for specified range of $x$. We use the convention $1 / \infty=0$.

\section{Estimates ON DISTRIBUtions OF SUbORDinATORS}

Let $S=\left(S_{t}\right)_{t \geq 0}$ be a driftless subordinator (i.e., a non-decreasing pure-jump Lévy process on $\mathbb{R}$ with $\left.S_{0}=0\right)$ with Laplace exponent $\phi$ given by

$$
\phi(\lambda)=-\log \mathbb{E} e^{-\lambda S_{1}}=\int_{0}^{\infty}\left(1-e^{-\lambda s}\right) \nu(d s) .
$$

Let $w(r):=\nu(r, \infty)$. Using that $\phi(\lambda)=\lambda \int_{0}^{\infty} e^{-\lambda s} w(s) d s$, it is easy to see (cf. the proof of [22, Lemma 2.1]) that

$$
e^{-1} \lambda \int_{0}^{1 / \lambda} w(s) d s \leq \phi(\lambda) \leq 2 \lambda \int_{0}^{1 / \lambda} w(s) d s, \quad \lambda>0
$$

The following is our main assumption on the subordinator $S$.

(Poly- $\left.R_{1}\right)$ There exist constants $R_{1} \in(0, \infty], c_{1}, c_{2}>0$ and $\beta_{2} \geq \beta_{1}>0$ such that

$$
c_{1}\left(\frac{R}{r}\right)^{\beta_{1}} \leq \frac{w(r)}{w(R)} \leq c_{2}\left(\frac{R}{r}\right)^{\beta_{2}} \text { for all } 0<r \leq R<R_{1} .
$$

Suppose that (Poly- $R_{1}$ ) holds. Then by [22, Lemma 2.1(ii)], in case $R_{1}<\infty$, for every $r_{0}>0$, there exists $c_{3}=c_{3}\left(r_{0}\right)>0$ such that

$$
\frac{\phi(R)}{\phi(r)} \leq c_{3}\left(\frac{R}{r}\right)^{\beta_{2} \wedge 1}, \quad r_{0}<r<R .
$$

On the other hand, by adapting the proof of [23, Lemma 2.3(3)], we can get that, for every $r_{0}>0$, there exists $c_{4}=c_{4}\left(r_{0}\right)>0$ such that

$$
\frac{\phi(R)}{\phi(r)} \geq c_{4}\left(\frac{R}{r}\right)^{\beta_{1}}, \quad r_{0}<r<R .
$$

As a consequence of (2.2) and (2.3), we see that $\beta_{1} \leq 1$ and that $\phi^{-1}$ enjoys the following scaling: For every $t_{0}>0$, there exist $c_{5}, c_{6}>0$ depending on $t_{0}$ such that

$$
c_{5}\left(\frac{t}{s}\right)^{1 /\left(\beta_{2} \wedge 1\right)} \leq \frac{\phi^{-1}(t)}{\phi^{-1}(s)} \leq c_{6}\left(\frac{t}{s}\right)^{1 / \beta_{1}}, \quad t_{0}<s<t .
$$

In case when (Poly- $\infty$ ) holds, (2.2) and (2.3) are valid for all $0<r<R$, and (2.4) is valid for all $0<s<t$.

Lemma 2.1. Assume (Poly- $\left.R_{1}\right)$ holds. For any $a>0$, there exists $c_{1}=c_{1}(a) \in(0,1)$ such that

$$
c_{1} \phi(\lambda) \leq \lambda \phi^{\prime}(\lambda) \leq \phi(\lambda), \quad \lambda>a .
$$

Further, if (Poly- $\infty)$ holds, then (2.5) holds for all $\lambda>0$. 
Proof. The second inequality follows from the fact $1-e^{-u}-u e^{-u} \geq 0$ for $u \geq 0$. The first inequality follows from [37, Lemma 1.3] and its proof.

Lemma 2.2. Assume $\left(\mathbf{P o l y}-R_{1}\right)$ holds. For any $a>0$, there exists $c_{1}=c_{1}(a)>0$ such that

$$
\left|\phi^{\prime \prime}(\lambda)\right| \leq c_{1} \lambda^{-1} \phi^{\prime}(\lambda), \quad \lambda>a .
$$

Further, if (Poly- $\infty)$ holds, then (2.6) holds for all $\lambda>0$.

Proof. The proof is similar to that of [23, Lemma 2.1(3)], where the existence of Lévy density is assumed. We give a detailed proof for the reader's convenience.

Since $e^{-x} \leq x^{-2}$ for all $x>0$, we see that for all $\lambda>1 / R_{1}$,

$$
\lambda\left|\phi^{\prime \prime}(\lambda)\right|=\int_{0}^{1 / \lambda} \lambda y^{2} e^{-\lambda y} \nu(d y)+\int_{1 / \lambda}^{\infty} \lambda y^{2} e^{-\lambda y} \nu(d y) \leq \int_{0}^{1 / \lambda} y \nu(d y)+\lambda^{-1} w(1 / \lambda) .
$$

By $\left(\right.$ Poly- $\left.R_{1}\right)$, there exists $\epsilon \in(0,1 / 2)$ such that $w(\epsilon / \lambda) \geq 2 w(1 / \lambda)$ for all $\lambda>1 / R_{1}$. Hence,

$$
\int_{0}^{1 / \lambda} y \nu(d y) \geq \epsilon \lambda^{-1} \int_{\epsilon / \lambda}^{1 / \lambda} \nu(d y) \geq \epsilon \lambda^{-1} w(1 / \lambda) \text { for all } \lambda>1 / R_{1} .
$$

It follows that

$$
\phi^{\prime}(\lambda) \geq e^{-1} \int_{0}^{1 / \lambda} y \nu(d y) \geq(2 e)^{-1} \epsilon\left(\int_{0}^{1 / \lambda} y \nu(d y)+\lambda^{-1} w(1 / \lambda)\right) .
$$

Combining (2.8) with (2.7), we get that in the case $R_{1}=\infty$, (2.6) holds for all $\lambda>0$ with $c_{1}=\epsilon /(2 e)$, and in the case $R_{1}<\infty$, (2.6) holds with

$$
c_{1}=\frac{\epsilon}{2 e} \vee \sup _{\lambda \in\left[a, 1 / R_{1}\right]}\left(\lambda\left|\phi^{\prime \prime}(\lambda)\right| / \phi^{\prime}(\lambda)\right) .
$$

Let $H:(0, \infty) \rightarrow(0, \infty)$ be defined by $H(\lambda):=\phi(\lambda)-\lambda \phi^{\prime}(\lambda), \lambda>0$. The function $H$ is strictly increasing, $H(0+)=0, \lim _{\lambda \rightarrow \infty} H(\lambda)=\int_{0}^{\infty} \nu(d s)=w(0+)$, and satisfies

$$
\frac{H(\lambda)}{\lambda^{2}}=-\left(\frac{\phi(\lambda)}{\lambda}\right)^{\prime}=\int_{0}^{\infty} e^{-\lambda s} s w(s) d s, \quad \lambda>0 .
$$

Since $1-e^{-\lambda s}-\lambda s e^{-\lambda} \geq 1-2 / e$ when $s \geq 1 / \lambda$, we see that

$$
\phi(\lambda) \geq H(\lambda) \geq \int_{1 / \lambda}^{\infty}\left(1-e^{-\lambda s}-\lambda s e^{-\lambda s}\right) \nu(d s) \geq \frac{e-2}{e} w(1 / \lambda), \quad \lambda>0 .
$$

Suppose that (Poly- $\left.R_{1}\right)$ holds. Then it follows from the proof of [23, Lemma 2.3(3)] that, for every $r_{0}>0$, there exists a constant $c=c\left(r_{0}\right)>0$ such that

$$
\frac{H(R)}{H(r)} \geq c\left(\frac{R}{r}\right)^{\beta_{1}}, \quad r_{0}<r \leq R
$$

As a consequence of (2.11), we have the following upper scaling for the inverse function $H^{-1}$ :

$$
\frac{H^{-1}(t)}{H^{-1}(s)} \leq c^{-1 / \beta_{1}}\left(\frac{t}{s}\right)^{1 / \beta_{1}}, \quad H\left(r_{0}\right)<s \leq t
$$

In case when $(\mathbf{P o l y}-\infty)$ holds, (2.11) and (2.12) hold with $r_{0}=0$. Note that (2.11) implies that $\lim _{\lambda \rightarrow \infty} H(\lambda)=+\infty$.

Next we look at the function $b:(0, \infty) \rightarrow(0, \infty)$ defined by

$$
b(t):=\left(\phi^{\prime} \circ H^{-1}\right)(1 / t)=\int_{0}^{\infty} s e^{-H^{-1}(1 / t) s} \nu(d s), \quad t>0 .
$$

The function $b$ is strictly increasing, $b(0+)=0$, and $\lim _{t \rightarrow \infty} b(t)=\int_{0}^{\infty} s \nu(d s)=\phi^{\prime}(0+)$. This implies that $t \mapsto t b(t)$ is also strictly increasing and $\lim _{t \rightarrow \infty} t b(t)=+\infty$. Moreover, according to [22, Lemma 2.4(ii)], cf. also [23, (2.13)], it holds that

$$
\phi^{-1}(7 / t)^{-1} \leq t b(t) \leq \phi^{-1}(1 / t)^{-1} \text { for all } t>0 .
$$


Hence, under (Poly- $\left.R_{1}\right)$, we see from the scaling of $\phi^{-1}$ in (2.4) that, for every $t_{0}>0$, there exists $c_{1}=c_{1}\left(t_{0}\right)>0$ such that

$$
c_{1} \phi^{-1}(1 / t)^{-1} \leq t b(t) \leq \phi^{-1}(1 / t)^{-1} \text { for all } 0<t<t_{0} .
$$

Moreover, if (Poly- $\infty$ ) holds, then (2.14) holds with $t_{0}=\infty$.

Finally, we introduce the function $\sigma=\sigma(t, s):(0, \infty) \times(0, \infty) \rightarrow[0, \infty)$ defined by

$$
\sigma=\sigma(t, s):=\left(\phi^{\prime}\right)^{-1}(s / t) \mathbf{1}_{\left(0, \phi^{\prime}(0+)\right)}(s / t), \quad s, t>0 .
$$

Note that $s \mapsto \sigma(t, s)$ is decreasing with $\lim _{s \rightarrow 0} \sigma(t, s)=\infty$ and $\lim _{s \rightarrow \infty} \sigma(t, s)=0$, while $t \mapsto \sigma(t, s)$ is increasing with $\lim _{t \rightarrow 0} \sigma(t, s)=0$ and $\lim _{t \rightarrow \infty} \sigma(t, s)=\infty$. Further, by using the former and the fact that $H$ is increasing, we conclude that

$$
t(H \circ \sigma)(t, t b(t))=1 \quad \text { and } \quad t(H \circ \sigma)(t, s)<1 \quad \text { for } s>t b(t) .
$$

The function $\sigma$ plays a crucial role in estimating the left tail of the subordinator $S$. We first state a result which follows from [22, Lemma 2.11] and [32, Lemma 5.2].

Proposition 2.3. There exist constants $c_{1}, c_{2}>0$ such that for all $t>0$,

$$
c_{1} \exp \left(-c_{2} t(H \circ \sigma)(t, s)\right) \leq \mathbb{P}\left(S_{t} \leq s\right) \leq e \exp (-t(H \circ \sigma)(t, s)) .
$$

Proof. If $s \leq t b(t)$, then it follows from [22, Lemma 2.11] and [32, Lemma 5.2] that there exist $c_{1}, c_{2}>0$ independent of $s$ and $t$ such that $c_{1} \exp \left(-c_{2} t(H \circ \sigma)(t, s)\right) \leq \mathbb{P}\left(S_{t} \leq s\right) \leq \exp (-t(H \circ \sigma)(t, s))$. (Note that the function $b(t)$ in this paper is the same as $t^{-1} b(t)$ in [22].) In particular, taking $s=t b(t)$ and using (2.15), we get $c_{3}:=c_{1} e^{-c_{2}} \leq \mathbb{P}\left(S_{t} \leq t b(t)\right) \leq e^{-1}$.

If $s>t b(t)$, then by the second part of (2.15), $\exp (-t(H \circ \sigma)(t, s)) \geq e^{-1}$, and thus $\mathbb{P}\left(S_{t} \leq s\right) \leq 1 \leq$ $e \exp (-t(H \circ \sigma)(t, s))$ which gives the desired upper bound. For the desired lower bound,

$$
\mathbb{P}\left(S_{t} \leq s\right) \geq \mathbb{P}\left(S_{t} \leq t b(t)\right) \geq c_{3} \geq c_{3} \exp \left(-c_{2} t(H \circ \sigma)(t, s)\right) .
$$

Lemma 2.4. Suppose (Poly- $\left.R_{1}\right)$ holds. Then, for any $a>0$, there exists $\delta=\delta(a)>0$ such that

$$
\frac{\sigma(t, u)}{\sigma(t, s)} \geq 2^{-\delta}\left(\frac{s}{u}\right)^{\delta}, \quad 0<u \leq s \leq t \phi^{\prime}(a) .
$$

Moreover, if (Poly- $\infty)$ holds, then (2.16) holds for all $0<u \leq s<t \phi^{\prime}(0+)$.

Proof. Let $a>0$. For all $0<2 w \leq t \phi^{\prime}(a)$, it holds that $\sigma(t, 2 w) \geq a$. By the mean value theorem, the fact that both $\left|\phi^{\prime \prime}\right|$ and $s \mapsto \sigma(t, s)$ are decreasing, and Lemma 2.2, we get

$$
\begin{aligned}
\frac{w}{t} & =\left(\phi^{\prime} \circ \sigma\right)(t, 2 w)-\left(\phi^{\prime} \circ \sigma\right)(t, w) \leq\left|\left(\phi^{\prime \prime} \circ \sigma\right)(t, 2 w)\right|(\sigma(t, w)-\sigma(t, 2 w)) \\
& \leq c_{1} \frac{\left(\phi^{\prime} \circ \sigma\right)(t, 2 w)}{\sigma(t, 2 w)}(\sigma(t, w)-\sigma(t, 2 w))=\frac{2 c_{1} w}{t} \frac{(\sigma(t, w)-\sigma(t, 2 w))}{\sigma(t, 2 w)} .
\end{aligned}
$$

Let $\delta=\log _{2}\left(1+1 /\left(2 c_{1}\right)\right)$. Then, we see from (2.17) that for all $0<2 w \leq t \phi^{\prime}(a)$,

$$
2^{\delta} \sigma(t, 2 w) \leq \sigma(t, w) .
$$

For any $0<u \leq s \leq t \phi^{\prime}(a)$, let $n=n(u, s)$ be the largest integer such that $2^{n} u \leq s$. Then, by (2.18), we obtain

$$
\frac{\sigma(t, u)}{\sigma(t, s)} \geq 2^{\delta n} \frac{\sigma\left(t, 2^{n} u\right)}{\sigma(t, s)} \geq 2^{\delta n} \geq 2^{\delta n} 2^{-\delta(n+1)}\left(\frac{s}{u}\right)^{\delta}=2^{-\delta}\left(\frac{s}{u}\right)^{\delta} .
$$

This proves the first assertion.

Assume now that $R_{1}=\infty$. Then (2.6) is valid for all $\lambda>0$ so that (2.17) holds for all $0<2 w<t \phi^{\prime}(0+)$. Hence, (2.18) holds for all $0<2 w<t \phi^{\prime}(0+)$. We conclude the proof as in the first assertion. 
Lemma 2.5. Suppose that (Poly- $\left.R_{1}\right)$ holds. Then, for all $\kappa, N>0$ and $T>0$, there exists a constant $C=C(T, \kappa, N)>0$ such that for all $0<t \leq T$ and $0<s \leq \phi^{-1}(1 / t)^{-1}$,

$$
\exp (-\kappa t(H \circ \sigma)(t, s)) \leq C\left(s \phi^{-1}(1 / t)\right)^{N} .
$$

Moreover, if $(\mathbf{P o l y}-\infty)$ holds, then for all $\kappa, N>0$, there exists a constant $C=C(\kappa, N)>0$ such that (2.19) holds for all $0<s \leq \phi^{-1}(1 / t)^{-1}$.

Proof. Choose an arbitrary $t \in(0, T]$. In view of (2.14), since $e^{-x} \leq 1$ for all $x \geq 0$, it suffices to prove (2.19) for $0<s \leq t b(t)$. Recall that $t(H \circ \sigma)(t, t b(t))=1$. Hence, by (2.11), Lemma 2.4 and (2.14), we have that, for all $0<s \leq t b(t)$,

$$
t(H \circ \sigma)(t, s)=\frac{(H \circ \sigma)(t, s)}{(H \circ \sigma)(t, t b(t))} \geq c_{1}\left(\frac{\sigma(t, s)}{\sigma(t, t b(t))}\right)^{\beta_{1}} \geq c_{2}\left(\frac{\phi^{-1}(1 / t)^{-1}}{s}\right)^{\delta \beta_{1}}
$$

where $\delta=\delta(T)$ is the constant from Lemma 2.4 Let $c_{3}:=\sup _{x>0} x^{N /\left(\delta \beta_{1}\right)} e^{-\kappa x}$. Then

$$
\exp (-\kappa t(H \circ \sigma)(t, s)) \leq c_{3}\left(\frac{1}{t(H \circ \sigma)(t, s)}\right)^{N /\left(\delta \beta_{1}\right)} \leq c_{2} c_{3}\left(s \phi^{-1}(1 / t)\right)^{N} .
$$

This proves the first assertion. Moreover, we can see that the second assertion is true by using that (2.12) and (2.14) hold for $r_{0}=0$ and $t_{0}=\infty$, respectively and the second assertion of Lemma 2.4.

Lemma 2.6. Let $f:(0, \infty) \rightarrow(0, \infty)$ be a given function. Assume that (Poly- $\left.R_{1}\right)$ holds and there exist constants $c_{1}, p>0$ such that $s^{p} f(s) \leq c_{1} t^{p} f(t)$ for all $0<s \leq t$. Then for every $T>0$, there exists a constant $C=C\left(T, c_{1}, p\right)>0$ such that for any $t \in(0, T]$,

$$
\mathbb{E}\left[f\left(S_{t}\right): S_{t} \leq r\right] \leq C f(r) \exp \left(-\frac{t}{2}(H \circ \sigma)(t, r)\right), \quad 0<r \leq \phi^{-1}(1 / t)^{-1} .
$$

Moreover, if (Poly- $\infty)$ holds, then there exists a constant $C=C\left(c_{1}, p\right)>0$ such that (2.20) holds for all $t>0$.

Proof. By using Proposition 2.3 in the second and Lemma 2.5 (with $\kappa=1 / 2$ and $N=p+1$ ) in the third inequality below, we get that

$$
\begin{aligned}
& \mathbb{E}\left[f\left(S_{t}\right): S_{t} \leq r\right]=\sum_{j=0}^{\infty} \int_{2^{-j-1} r}^{2^{-j} r} f(s) \mathbb{P}\left(S_{t} \in d s\right) \leq c_{1} 2^{p} \sum_{j=0}^{\infty} f\left(2^{-j} r\right) \mathbb{P}\left(S_{t} \leq 2^{-j} r\right) \\
& \leq c_{1}^{2} 2^{p} f(r) \sum_{j=0}^{\infty} 2^{j p} \exp \left(-\frac{t}{2}(H \circ \sigma)\left(t, 2^{-j} r\right)\right) \exp \left(-\frac{t}{2}(H \circ \sigma)\left(t, 2^{-j} r\right)\right) \\
& \leq c_{2} f(r) \exp \left(-\frac{t}{2}(H \circ \sigma)(t, r)\right) \sum_{j=0}^{\infty} 2^{j p}\left(2^{-j} r \phi^{-1}(1 / t)\right)^{p+1} \leq 2 c_{2} f(r) \exp \left(-\frac{t}{2}(H \circ \sigma)(t, r)\right) .
\end{aligned}
$$

Proposition 2.7. Suppose that (Poly- $\left.R_{1}\right)$ holds. Then for any $T>0$, there exist constants $\delta \in(0,1)$ independent of $T$ and $\epsilon=\epsilon(T) \in(0,1)$ such that

$$
\mathbb{P}\left(\epsilon \phi^{-1}(1 / t)^{-1} \leq S_{t} \leq \phi^{-1}(1 / t)^{-1}\right) \geq \delta, \quad t \in(0, T) .
$$

Moreover, if (Poly- $\infty)$ holds, then there exist $\epsilon, \delta \in(0,1)$ such that (2.21) holds with $T=\infty$.

Proof. By Proposition 2.3, (2.15) and (2.14), there exists a constant $c_{1} \in(0,1)$ such that $\mathbb{P}\left(S_{t} \leq\right.$ $\left.\phi^{-1}(1 / t)^{-1}\right) \geq c_{1}$ for all $t>0$. Let $c_{2}=\log \left(2 / c_{1}\right)$. Then, using Proposition 2.3 again, we get that for all $t>0$

$$
\mathbb{P}\left(t b\left(t / c_{2}\right) \leq S_{t} \leq \phi^{-1}(1 / t)^{-1}\right) \geq c_{1}-\mathbb{P}\left(S_{t}<t b\left(t / c_{2}\right)\right) \geq c_{1}-e^{-c_{2}}=c_{1} / 2 .
$$

Since (Poly- $\left.R_{1}\right)$ holds, by (2.14) and (2.4), there exists $\epsilon \in(0,1)$ such that

$$
t b\left(t / c_{2}\right) \geq \epsilon \phi^{-1}(1 / t)^{-1}, \quad t \in(0, T) .
$$

We also see that if (Poly- $\infty$ ) holds, then (2.23) holds with $T=\infty$. Combining this with (2.22), we obtain (2.21). 
Lemma 2.8. Suppose that (Poly- $\left.R_{1}\right)$ holds. Then, for any $\kappa>0$, there exists a constant $a_{1}=a_{1}(\kappa)>0$ such that for all $\phi^{-1}(1 / t)^{-1} \leq s<R_{1} / 2$, we have $\exp \left(-\kappa s H^{-1}(1 / t)\right) \leq a_{1} t w(s)$.

Proof. According to [22, Lemma 2.2], there exists $c_{1}>0$ such that $H(1 / s)^{1+\beta_{2}} \leq c_{1} \phi(1 / s)^{\beta_{2}} w(s)$, $s \in\left(0, R_{1} / 2\right]$. Note that by (2.9), the map $\lambda \rightarrow \lambda^{-2} H(\lambda)$ is decreasing. Let $c_{2}:=\sup _{x>0} x^{2\left(1+\beta_{2}\right)} e^{-\kappa x}$. Then by using (Poly- $\left.R_{1}\right)$ and the fact that $\phi$ is increasing, we get that for all $\phi^{-1}(1 / t)^{-1} \leq s<R_{1} / 2$, since $1 / s \leq \phi^{-1}(1 / t) \leq H^{-1}(1 / t)$,

$$
\begin{aligned}
& \exp \left(-\kappa s H^{-1}(1 / t)\right) \leq c_{2}\left(\frac{1 / s}{H^{-1}(1 / t)}\right)^{2\left(1+\beta_{2}\right)} \leq c_{2}\left(\frac{H(1 / s)}{1 / t}\right)^{1+\beta_{2}} \\
& \leq c_{1} c_{2} t^{1+\beta_{2}} \phi(1 / s)^{\beta_{2}} w(s) \leq c_{1} c_{2} t^{1+\beta_{2}} \phi\left(\phi^{-1}(1 / t)\right)^{\beta_{2}} w(s)=c_{1} c_{2} t w(s) .
\end{aligned}
$$

This proves the lemma.

Proposition 2.9. Suppose that (Poly- $\left.R_{1}\right)$ holds. Then, for all $2 \phi^{-1}(1 / t)^{-1}<s<R_{1} / 2$,

$$
\mathbb{P}\left(S_{t} \geq s\right) \simeq t w(s) .
$$

In particular, there exists a constant $M>1$ such that for all $2 \phi^{-1}(1 / t)^{-1}<s<R_{1} /(2 M)$,

$$
\mathbb{P}\left(S_{t} \in[s, M s]\right) \simeq t w(s) .
$$

Proof. The lower bound of (2.24) follows from [22, Lemma 2.6] (note that $\left.t \phi\left(s^{-1}\right) \leq 1\right)$. The upper bound of (2.24) comes from the proof of [22, Proposition 2.7] with a bit of modification. We provide most of the proof for the reader's convenience.

Pick an arbitrary $s \in\left(2 \phi^{-1}(1 / t)^{-1}, R_{1} / 2\right)$. Let $\epsilon=\log (5 / 4) / 2 \in(0,1)$. We set

$$
\mu^{1}:=\mathbf{1}_{\left(0, \epsilon / H^{-1}(1 / t)\right]} \nu(d x), \quad \mu^{2}:=\mathbf{1}_{\left(\epsilon / H^{-1}(1 / t), s\right]} \nu(d x) \text { and } \mu^{3}:=\mathbf{1}_{(s, \infty)} \nu(d x)
$$

and denote by $S^{1}, S^{2}$ and $S^{3}$ the independent driftless subordinators with Lévy measures $\mu^{1}, \mu^{2}$ and $\mu^{3}$, respectively. Then $S_{t} \leq S_{t}^{1}+S_{t}^{2}+S_{t}^{3}$ (note that it may happen that $s<\epsilon / H^{-1}(1 / t)$ ) and hence

$$
\mathbb{P}\left(S_{t} \geq s\right) \leq \mathbb{P}\left(S_{t}^{1} \geq 3 s / 4\right)+\mathbb{P}\left(S_{t}^{2} \geq s / 4\right)+\mathbb{P}\left(S_{t}^{3}>0\right) .
$$

Since $S^{3}$ is a compound Poisson process, it holds that $\mathbb{P}\left(S_{t}^{3}>0\right)=1-e^{-t w(s)} \leq t w(s)$. Moreover, by following the proof of [22, Proposition 2.7], one can obtain from [34, Proposition 1 and Lemma 9] that $\mathbb{P}\left(S_{t}^{2} \geq s / 4\right) \leq c t w(s)$. Lastly, by using Markov's inequality and [22, Lemma 2.5], since $s>2 t b(t)$ due to (2.13), we have that

$$
\begin{aligned}
\mathbb{P}\left(S_{t}^{1} \geq 3 s / 4\right) & \leq \mathbb{E}\left[\exp \left(-(3 s / 4) H^{-1}(1 / t)+H^{-1}(1 / t) S_{t}^{1}\right)\right] \\
& =\exp \left(-(3 s / 4) H^{-1}(1 / t)+t \int_{0}^{\epsilon / H^{-1}(1 / t)}\left(e^{H^{-1}(1 / t) x}-1\right) \nu(d x)\right) \\
& \leq \exp \left(-(3 s / 4) H^{-1}(1 / t)+e^{2 \epsilon} t H^{-1}(1 / t) \int_{0}^{\epsilon / H^{-1}(1 / t)} x e^{-H^{-1}(1 / t) x} \nu(d x)\right) \\
& \leq \exp \left(-(3 s / 4) H^{-1}(1 / t)+(5 / 4) H^{-1}(1 / t) t b(t)\right) \leq \exp \left(-2^{-3} s H^{-1}(1 / t)\right) .
\end{aligned}
$$

We used the fact that $e^{y}-1 \leq y e^{-y} e^{2 y}$ for all $y \geq 0$ in the third line. Hence, by Lemma 2.8, we get that $\mathbb{P}\left(S_{t}^{1} \geq 3 s / 4\right) \leq c t w(s)$ and hence the first assertion holds.

The second assertion follows from (Poly- $\left.R_{1}\right)$.

\section{SEtup AND MAin ASSUmptions}

Let $(E, \rho)$ be a locally compact separable metric space such that all bounded closed sets are compact, and let $m$ a positive Radon measure on $E$ with full support. We use $B(x, r)$ to denote the open ball in $(E, \rho)$ of radius $r$ centered at $x$, and $V(x, r):=m(B(x, r))$ its volume.

Throughout the remainder of this paper, we assume the following volume doubling and reverse volume doubling properties with localization radius $R_{E} \in(0, \infty]$ : There exist constants $d_{2} \geq d_{1}>0$ such that, for every $a \geq 1$, there exists a constant $C_{V}=C_{V}(a) \geq 1$ satisfying

$$
C_{V}^{-1}\left(\frac{R}{r}\right)^{d_{1}} \leq \frac{V(x, R)}{V(x, r)} \leq C_{V}\left(\frac{R}{r}\right)^{d_{2}} \quad \text { for all } x \in E \text { and } 0<r \leq R<a R_{E} .
$$


As a consequence of (3.1), we see that for all $R_{0}, \epsilon, \eta>0$, there exists a constant $C=C\left(R_{0}, \epsilon, \eta\right)>0$ such that

$$
V(x, r) \leq C V(y, \eta r) \quad \text { for all } x, y \in E \text { and } \epsilon \rho(x, y)<r \leq R_{0} .
$$

Indeed, since $B(x, r) \subset B(y, r+\rho(x, y))$, we get from (3.1) that $V(x, r) \leq V(y, r+\rho(x, y)) \leq V(y,(1+$ $1 / \epsilon) r) \leq c_{1} V(y, \eta r)$. Moreover, if the localization radius $R_{E}$ is infinite, then the above constant $C$ is independent of $R_{0}$ and (3.2) holds for $\epsilon \rho(x, y)<r<\infty$.

Let $D$ be a proper open subset of $E$. We use $\operatorname{diam}(D)$ to denote the diameter of $D$. If $\operatorname{diam}(D)<\infty$, i.e., $D$ is bounded, then by the assumption on $E$ it holds that $m(D)<\infty$. For $x \in D$, let $\delta_{D}(x)=\rho(x, E \backslash D)$. In most applications, $D$ will be an open subset of the Euclidean space $\mathbb{R}^{d}, d \geq 1$, and $m(d y)$ will be the Lebesgue measure. For simplicity we write $d y$ instead of $m(d y)$. Let $Y^{D}=\left(Y_{t}^{D}, \mathbb{P}^{x}\right)$ be a Hunt process in $D$. We assume that the semigroup of $Y^{D}$ admits a density $p_{D}(t, x, y)$, which we call the heat kernel of $Y^{D}$. Thus, for any non-negative Borel function $f$ on $D$,

$$
\mathbb{E}^{x}\left[f\left(Y_{t}^{D}\right)\right]=\int_{D} f(y) p_{D}(t, x, y) d y .
$$

Let $S=\left(S_{t}\right)_{t \geq 0}$ be a driftless subordinator independent of $Y^{D}$. We will be interested in the subordinate process $X_{t}:=Y_{S_{t}}^{D}$. It is well known (cf. [6, p.67, pp. 73-75] and [50]) that $X$ is also a Hunt process and admits a heat kernel $q(t, x, y)$ which is given by the formula

$$
q(t, x, y)=\mathbb{E}\left[p_{D}\left(S_{t}, x, y\right)\right]=\int_{0}^{\infty} p_{D}(s, x, y) \mathbb{P}\left(S_{t} \in d s\right) .
$$

Our goal is to find two-sided estimates of $q(t, x, y)$ under certain assumptions on the underlying heat kernel $p_{D}(t, x, y)$ and the subordinator $S$. On the subordinator we will impose the assumption (Poly- $\left.R_{1}\right)$. Now we explain the assumptions we impose on $p_{D}(t, x, y)$. These assumptions are motivated by various examples from the literature.

We first introduce two functions $\Phi, \Psi:[0, \infty) \rightarrow[0, \infty)$, both strictly increasing and satisfying $\Psi(r) \geq$ $\Phi(r)$ for all $r \geq 0$. Moreover, we always assume that both satisfy global scaling conditions: There exist constants $\alpha_{1}, \alpha_{2}, \alpha_{3}, \alpha_{4}>0$ and $c_{1}, c_{2}, c_{3}, c_{4}>0$ such that for all $R \geq r>0$,

$$
c_{1}\left(\frac{R}{r}\right)^{\alpha_{1}} \leq \frac{\Phi(R)}{\Phi(r)} \leq c_{2}\left(\frac{R}{r}\right)^{\alpha_{2}} \quad \text { and } \quad c_{3}\left(\frac{R}{r}\right)^{\alpha_{3}} \leq \frac{\Psi(R)}{\Psi(r)} \leq c_{4}\left(\frac{R}{r}\right)^{\alpha_{4}} .
$$

As an easy consequence we see that, for every $a \geq 1$, there exist two constants $c_{1}(a)>0$ and $c_{2}(a)>0$ such that, for all $r, R>0$ satisfying $0<r \leq a R$, it holds that

$$
c_{1}(a)\left(\frac{R}{r}\right)^{\alpha_{1}} \leq \frac{\Phi(R)}{\Phi(r)} \leq c_{2}(a)\left(\frac{R}{r}\right)^{\alpha_{2}} .
$$

The following lemma shows that, without loss of generality, we may replace $\Phi$ by a nicer function.

Lemma 3.1. There exists a strictly increasing differentiable functions $\widetilde{\Phi}$ satisfying the following two properties:

$(P 1) \Phi(r) \simeq \widetilde{\Phi}(r)$ for all $r>0$ and $\widetilde{\Phi}$ satisfies (3.5);

$($ P $) ~ \widetilde{\Phi}^{\prime}(r) \simeq r^{-1} \widetilde{\Phi}(r)$ and $\left(\widetilde{\Phi}^{-1}\right)^{\prime}(t) \simeq t^{-1} \widetilde{\Phi}^{-1}(t)$ for $r, t>0$.

Proof. According to [8, Lemmas 3.1 and 3.2], for any $\alpha>\alpha_{2}$, there exists a complete Bernstein function $\varphi$ such that

$$
\Phi(r) \simeq \varphi\left(r^{-\alpha}\right)^{-1} \quad \text { and } \quad \varphi^{\prime}(r) \simeq r^{-1} \varphi(r) \text { for all } r>0,
$$

and that $\varphi$ satisfies the weak scaling conditions with exponents $\alpha_{1} / \alpha$ and $\alpha_{2} / \alpha$. Let $\widetilde{\Phi}(r):=\varphi\left(r^{-\alpha}\right)^{-1}$, $r>0$. It is straightforward to check that $\widetilde{\Phi}$ satisfies (3.5) and also that $\widetilde{\Phi}^{\prime}(r) \simeq r^{-1} \widetilde{\Phi}(r)$. Moreover, by the inverse function theorem, the second comparability in (P2) is also valid.

Lemma 3.2. Let $f:(0, \infty) \rightarrow(0, \infty)$ be a given function. Assume that there exist constants $c_{1}, p>0$ such that $s^{p} f(s) \leq c_{1} t^{p} f(t)$ for all $0<s \leq t$. Then there exists a constant $c_{2}=c_{2}\left(c_{1}, p\right)>0$ such that for all $r, \kappa>0$,

$$
\int_{0}^{r} f(s) \exp \left(-\frac{\kappa^{2}}{\Phi^{-1}(s)^{2}}\right) d s \leq \frac{c_{2} r^{p+1} f(r)}{\Phi(\kappa)^{p}} .
$$


Proof. Let $c_{3}:=\sup _{u>0} u^{p \alpha_{2} / 2} e^{-u}$. Then by the scaling of $\Phi$, we have that

$$
\begin{aligned}
& \int_{0}^{r} f(s) \exp \left(-\frac{\kappa^{2}}{\Phi^{-1}(s)^{2}}\right) d s \leq c_{3} \int_{0}^{r \wedge \Phi(\kappa)} f(s)\left(\frac{\Phi^{-1}(s)}{\kappa}\right)^{p \alpha_{2}} d s+\int_{r \wedge \Phi(\kappa)}^{r} f(s) d s \\
& \leq c_{4} \int_{0}^{r \wedge \Phi(\kappa)} f(s)\left(\frac{s}{\Phi(\kappa)}\right)^{p} d s+\int_{r \wedge \Phi(\kappa)}^{r} s^{-p} s^{p} f(s) d s \\
& \leq \frac{c_{1} c_{4} r^{p} f(r)}{\Phi(\kappa)^{p}} \int_{0}^{r} d s+\frac{c_{1} r^{p} f(r)}{(r \wedge \Phi(\kappa))^{p}} \int_{r \wedge \Phi(\kappa)}^{r} d s \leq \frac{c_{1}\left(c_{4}+1\right) r^{p+1} f(r)}{\Phi(\kappa)^{p}} .
\end{aligned}
$$

Definition 3.3. We say that a function $h:(0, \infty) \times D \times D \rightarrow[0,1]$ is a boundary function if it satisfies the following two properties:

(H1) For all fixed $x, y \in D$, the map $s \mapsto h(s, x, y)$ is non-increasing.

(H2) There exist constants $c_{1}>0, \gamma \geq 0$ such that

$$
s^{\gamma} h(s, x, y) \leq c_{1} t^{\gamma} h(t, x, y), \quad 0<s \leq t<4 \Phi(\operatorname{diam}(D))+1, x, y \in D,
$$

with $4 \Phi(\operatorname{diam}(D))+1$ interpreted as $\infty$ when $D$ is unbounded.

A boundary function $h$ is said to be regular if there exists $c_{2}>0$ such that

$$
h(t, x, y) \geq c_{2}, \quad 0<t<4 \Phi(\operatorname{diam}(D))+1, x, y \in D \text { with } \delta_{\wedge}(x, y) \geq \Phi^{-1}(t) .
$$

A regular boundary function $h$ is said to be of Harnack-type if there exists $c_{3}>0$ such that for all $x, y, z \in D$ satisfying $\rho(x, z) \leq\left(\rho(x, y) \wedge \delta_{D}(x)\right) / 2$,

$$
h(t, x, y) \leq c_{3} h(t, z, y), \quad 0<t<\Phi(\rho(x, y)) .
$$

From now on, $h(t, x, y)$ always denotes a boundary function.

Remark 3.4. Suppose that $h$ is a regular boundary function. Then for every $\epsilon \in(0,1)$, there exists $c_{1}=c_{1}(\epsilon)>0$ such that

$$
h(t, x, y) \geq c_{1}, \quad 0<t<4 \Phi(\operatorname{diam}(D))+1, x, y \in D \text { with } \delta_{\wedge}(x, y) \geq \epsilon \Phi^{-1}(t) .
$$

Indeed, by (3.4) and (H2), we see that for all $x, y \in D$ with $\delta_{\wedge}(x, y) \geq \epsilon \Phi^{-1}(t)$,

$$
h(t, x, y) \geq c_{2} h\left(\Phi\left(\epsilon \Phi^{-1}(t)\right), x, y\right) \geq c_{3} .
$$

Example 3.5. (a) Let $p, q \geq 0$. For $t>0$ and $x, y \in D$, define

$$
h_{p, q}(t, x, y):=\left(1 \wedge \frac{\Phi\left(\delta_{D}(x)\right)}{t}\right)^{p}\left(1 \wedge \frac{\Phi\left(\delta_{D}(y)\right)}{t}\right)^{q}, \quad h_{p}(t, x, y):=h_{p, p}(t, x, y) .
$$

Then $h_{p, q}(t, x, y)$ is a typical example of a regular boundary function which is also of Harnack-type. Indeed, (H1) and the regularity is clear, while (H2) holds with $c_{1}=1$ and $\gamma=p+q$ since for all $0<s<t$,

$$
t^{p+q} h_{p, q}(t, x, y)=\left(t \wedge \Phi\left(\delta_{D}(x)\right)\right)^{p}\left(t \wedge \Phi\left(\delta_{D}(y)\right)\right)^{q} \geq s^{p+q} h_{p, q}(s, x, y) .
$$

Moreover, we see from (3.4) that for all $x, y, z \in D$ satisfying $\rho(x, z) \leq\left(\rho(x, y) \wedge \delta_{D}(x)\right) / 2$, since $\delta_{D}(z) \geq$ $\delta_{D}(x)-\rho(x, z) \geq \delta_{D}(x) / 2$,

$$
\left(1 \wedge \frac{\Phi\left(\delta_{D}(x)\right)}{t}\right)^{p} \leq\left(1 \wedge \frac{\Phi\left(2 \delta_{D}(z)\right)}{t}\right)^{p} \leq c_{1}\left(1 \wedge \frac{\Phi\left(\delta_{D}(z)\right)}{t}\right)^{p}
$$

Thus we conclude that $h_{p, q}(t, x, y)$ is of Harnack-type. The boundary function $h_{p}(t, x, y)$ is very typical when $D$ is a bounded smooth open subset of $\mathbb{R}^{d}$.

(b) Let $h_{p}(t, x, y)$ be the function defined in (3.8). Then $h_{p}(t \wedge 1, x, y)$ is also a regular boundary function of Harnack-type. This is a typical boundary function for smooth exterior open sets.

(c) A quite general example of a boundary function is obtained as follows. Suppose that $Y^{D}$ admits a dual process $\widehat{Y}^{D}$. Let $\zeta$ and $\widehat{\zeta}$ be the lifetimes of $Y^{D}$ and $\widehat{Y}^{D}$ respectively. Assume that the survival probabilities $\mathbb{P}^{x}(\zeta>t)$ and $\mathbb{P}^{y}(\widehat{\zeta}>t)$ satisfy the following doubling property: $\mathbb{P}^{x}(\zeta>t / 2) \simeq \mathbb{P}^{x}(\zeta>t)$ and $\mathbb{P}^{y}(\widehat{\zeta}>t / 2) \simeq \mathbb{P}^{y}(\widehat{\zeta}>t)$ for all $0<t<4 \Phi(\operatorname{diam}(D))+1$ and $x, y \in D$. Then $h(t, x, y):=\mathbb{P}^{x}(\zeta>$ $t) \mathbb{P}^{y}(\widehat{\zeta}>t)$ is a boundary function. Indeed, (H1) is clear, while (H2) follows from the doubling property 
of survival probabilities assumed above. The survival probabilities usually satisfy the doubling property, see for instance, [25, Lemma 2.21] and its proof. In fact, by [25, Lemma 2.21] and its proof, one can see that the boundary function $h$ above is often regular.

Moreover, the above $h(t, x, y)$ is of Harnack-type if, in addition, (1) it is regular; (2) $Y^{D}$ satisfies the (interior elliptic) Harnack inequality and (3) there is $c_{1}>0$ such that for all $x \in D$ and $\Phi\left(\delta_{D}(x)\right)<t<$ $\Phi(\operatorname{diam}(D))$,

$$
\mathbb{P}^{x}(\zeta>t) \simeq \mathbb{P}^{x}\left(\zeta>\tau_{U(x, t)}\right)=\mathbb{P}^{x}\left(Y_{\tau_{U(x, t)}}^{D}, \in D\right),
$$

where $U(x, t):=B\left(x, c_{1} \Phi^{-1}(t)\right) \cap D$ and $\tau_{V}=\inf \left\{t>0: Y_{t}^{D} \notin V\right\}$.

To see this, we fix $x, z \in D$ satisfying $\rho(x, z) \leq \delta_{D}(x) / 2$. If $\delta_{D}(x) \vee \delta_{D}(z) \geq\left(c_{1} \wedge 2^{-1}\right) \Phi^{-1}(t)$, then we have $\delta_{D}(x) \wedge \delta_{D}(z) \geq \delta_{D}(x) \vee \delta_{D}(z)-2^{-1} \delta_{D}(x) \geq 2^{-1}\left(c_{1} \wedge 2^{-1}\right) \Phi^{-1}(t)$. By Remark 3.4, it follows that $1 \geq \mathbb{P}^{x}(\zeta>t) \wedge \mathbb{P}^{z}(\zeta>t) \geq h(t, x, x) \wedge h(t, z, z) \geq c_{2}$. Hence, we obtain $h(t, x, y) / h(t, z, y)=$ $\mathbb{P}^{x}(\zeta>t) / \mathbb{P}^{z}(\zeta>t) \leq 1 / c_{2}$. If $\delta_{D}(x) \vee \delta_{D}(z)<\left(c_{1} \wedge 2^{-1}\right) \Phi^{-1}(t)$, then $B\left(x, \delta_{D}(x)\right) \subset U(x, t)$ so that $v \mapsto \mathbb{P}^{v}\left(Y_{\tau_{U(x, t)}^{D}}^{D} \in D\right)$ is harmonic in $B\left(x, \delta_{D}(x)\right)$ with respect to $Y^{D}$. Using (3.9) twice, we see from the Harnack inequality, (3.4) and (H2) that

$$
\mathbb{P}^{x}(\zeta>t) \leq c_{3} \mathbb{P}^{z}\left(Y_{\tau_{U(x, t)}}^{D} \in D\right) \leq c_{3} \mathbb{P}^{z}\left(Y_{\tau_{U\left(z, \Phi\left(\Phi^{-1}(t) / 2\right)\right)}^{D}}^{D} \in D\right) \leq c_{4} \mathbb{P}^{z}\left(\zeta>\Phi\left(\Phi^{-1}(t) / 2\right)\right) \leq c_{5} \mathbb{P}^{z}(\zeta>t) .
$$

The second inequality above is valid since $U\left(z, \Phi\left(\Phi^{-1}(t) / 2\right)\right) \subset U(x, t)$. Therefore, we obtain (3.7).

Under the setting and assumptions in [25, Section 2] (Assumptions $\mathbf{A}$ and $\mathbf{U}$ in [25]), for the Hunt process $Y$ defined right below [25, (2.27)] on a $\kappa$-fat open set $D$ with a critical killing potential $\mu \in \mathbf{K}_{1}(D)$, by [25. Lemma 2.21], we know that the boundary function $h(t, x, y)=\mathbb{P}^{x}(\zeta>t) \mathbb{P}^{y}(\widehat{\zeta}>t)$ is of Harnacktype. (See [25, Definition 2.19] and [25, Definition 2.12] for the definitions of a $\kappa$-fat open set and the class $\mathbf{K}_{1}(D)$, respectively.) See [2, 4, 14, for related work.

For later use, we record the following simple consequence of (H1) and (H2): Let $k>1$ and $s, t>0$ satisfy $k^{-1} s \leq t \leq k s \leq 4 \Phi(\operatorname{diam}(D))$. Then for all $x, y \in D$,

$$
c_{1}^{-1} k^{-\gamma} h(s, x, y) \leq h(t, x, y) \leq c_{1} k^{\gamma} h(s, x, y)
$$

where $c_{1}$ is the constant from (H2).

Definition 3.6. Let $h(t, x, y)$ be a boundary function.

(a) We say that $\mathbf{H K}_{\mathbf{B}}^{\mathbf{h}}$ holds, if $D$ is bounded and the following estimates hold: (i) there exist $C_{0} \in\{0,1\}$ and $c_{1}, c_{2}, c_{3}, c_{4}>0$ such that for all $(t, x, y) \in(0,1] \times D \times D$,

$$
\begin{aligned}
& c_{1} h(t, x, y)\left[\frac{1}{V\left(x, \Phi^{-1}(t)\right)} \wedge\left(\frac{C_{0} t}{V(x, \rho(x, y)) \Psi(\rho(x, y))}+\frac{1}{V\left(x, \Phi^{-1}(t)\right)} \exp \left(-\frac{c_{2} \rho(x, y)^{2}}{\Phi^{-1}(t)^{2}}\right)\right)\right] \\
& \leq p_{D}(t, x, y) \\
& \leq c_{3} h(t, x, y)\left[\frac{1}{V\left(x, \Phi^{-1}(t)\right)} \wedge\left(\frac{C_{0} t}{V(x, \rho(x, y)) \Psi(\rho(x, y))}+\frac{1}{V\left(x, \Phi^{-1}(t)\right)} \exp \left(-\frac{c_{4} \rho(x, y)^{2}}{\Phi^{-1}(t)^{2}}\right)\right)\right],
\end{aligned}
$$

and (ii) there exists a constant $\lambda_{D}>0$ such that for all $(t, x, y) \in[1, \infty) \times D \times D$,

$$
p_{D}(t, x, y) \simeq e^{-\lambda_{D} t} h(1, x, y) .
$$

(b) We say that $\mathbf{H K}_{\mathbf{U}}^{\mathbf{h}}$ holds, if the constant $R_{E}$ in (3.1) is infinite and (3.11) holds for all $(t, x, y) \in$ $(0, \infty) \times D \times D$.

By using the function $\left(1 \wedge \frac{R_{1}}{10 \Phi(\operatorname{diam}(D))}\right) \Phi(r)$ instead of $\Phi(r)$, we may and do assume that $\Phi(\operatorname{diam}(D))<$ $R_{1} / 8$ whenever (Poly- $\left.R_{1}\right)$ and $\mathbf{H} K_{\mathrm{B}}^{\mathbf{h}}$ hold.

Remark 3.7. One can easily see that if $\mathbf{H K}_{\mathbf{B}}^{\mathbf{h}}$ holds, then for every $T>0$, there exist constants $c_{1}, c_{2}, c_{3}, c_{4}>0$ such that (3.11) holds for all $(t, x, y) \in(0, T] \times D \times D$, and (3.12) holds for all $(t, x, y) \in$ $[T, \infty) \times D \times D$.

Remark 3.8. Note that $a \wedge(b+c) \leq(a \wedge b)+(a \wedge c) \leq 2(a \wedge(b+c))$ for all $a, b, c>0$. Hence (3.11) is equivalent to the statement that for all $(t, x, y) \in(0,1] \times D \times D$,

$$
p_{D}(t, x, y) \asymp h(t, x, y)\left[\left(\frac{1}{V\left(x, \Phi^{-1}(t)\right)} \wedge \frac{C_{0} t}{V(x, \rho(x, y)) \Psi(\rho(x, y))}\right)+\frac{1}{V\left(x, \Phi^{-1}(t)\right)} \exp \left(-\frac{c \rho(x, y)^{2}}{\Phi^{-1}(t)^{2}}\right)\right] .
$$


Example 3.9. Here are several examples of processes satisfying $\mathbf{H} \mathbf{K}_{\mathbf{B}}^{\mathbf{h}}$ or $\mathbf{H} \mathbf{K}_{\mathbf{U}}^{\mathbf{h}}$. We will not try to give the most general examples but the reader will see from examples below that our setup is general enough to cover almost all known cases. In all examples below, the boundary functions are of Harnack type.

(a) Suppose that $D$ is a bounded $C^{1,1}$ open subset of $\mathbb{R}^{d}$.

(1) If $D$ is connected and $Y^{D}$ is the killed Brownian motion in $D$, then $\mathbf{H K}_{\mathbf{B}}^{\mathbf{h}}$ is satisfied with $C_{0}=0$, $\Phi(r)=r^{2}$ and boundary function $h_{1 / 2}$. See [24] for a more general example.

(2) If $\alpha \in(0,2)$ and $Y^{D}$ is a killed isotropic $\alpha$-stable process in $D$, then $\mathbf{H K}_{\mathbf{B}}^{\mathbf{h}}$ is satisfied with $\Phi(r)=\Psi(r)=r^{\alpha}$ and boundary function $h_{1 / 2}$, cf. [9]. More generally, suppose $\chi$ is a complete Bernstein function satisfying global weak scaling conditions with indices $\beta_{1}, \beta_{2} \in(0,1), Y$ is a subordinate Brownian motion in $\mathbb{R}^{d}$ via an independent subordinator with Laplace exponent $\chi, Y^{D}$ is the part process of $Y$ in $D$. Then $\mathbf{H K}_{\mathbf{B}}^{\mathbf{h}}$ is satisfied with $\Phi(r)=\Psi(r)=1 / \chi\left(r^{-2}\right)$ and boundary function $h_{1 / 2}$, cf. [14]. See [3, 31, 36] for more general examples.

(3) If $D$ is connected and $Y$ is the independent sum of isotropic $\alpha$-stable process and Brownian motion, then its part process $Y^{D}$ in $D$ satisfies $\mathbf{H K}_{\mathbf{B}}^{\mathbf{h}}$ with $\Phi(r)=r^{2} \wedge r^{\alpha}, \Psi(r)=r^{\alpha}$ and boundary function $h_{1 / 2}$, cf. [11]. More generally, suppose $\chi$ is a complete Bernstein function satisfying the conditions in the paragraph above and $Y$ is the independent sum of Brownian motion and a subordinate Brownian motion via a subordinator with Laplace exponent $\chi$, then its part process $Y^{D}$ in $D$ satisfies $\mathbf{H K}_{\mathbf{B}}^{\mathbf{h}}$ with $\Phi(r)=\Phi_{\chi}(r):=r^{2} \wedge\left(1 / \chi\left(r^{-2}\right)\right), \Psi(r)=1 / \chi\left(r^{-2}\right)$ and boundary function $h_{1 / 2}$, cf. [15]. Note that since $\lim _{\lambda \rightarrow \infty} \chi(\lambda) / \lambda=0$ (see (2.1)), for every $a>0$, there are comparability constants depending on $a$ such that $\Phi_{\chi}(r) \simeq r^{2}$ for $r \in(0, a)$. We remark here that the estimates in [11, (1.4)] and [15, (1.14)] are comparable to (3.13) since $t \leq 1$.

(4) Suppose that $\chi$ is a complete Bernstein function such that the function $\lambda \mapsto \chi(\lambda)-\lambda \chi^{\prime}(\lambda)$ satisfies weak scaling conditions for $\lambda \geq a>0$ with upper index $\delta<2$ and lower index $\gamma>2^{-1} \mathbf{1}_{\{\delta \geq 1\}}$. Suppose that $Y$ is a subordinate Brownian motion in $\mathbb{R}^{d}$ via an independent subordinator with Laplace exponent $\chi, Y^{D}$ is the part process of $Y$ in $D$. Then $\mathbf{H K}_{\mathbf{B}}^{\mathbf{h}}$ is satisfied with $\Phi(r)=1 / \chi\left(r^{-2}\right), \Psi(r)=1 /\left(\chi\left(r^{-2}\right)-\right.$ $\left.r^{-2} \chi^{\prime}\left(r^{-2}\right)\right)$ and boundary function $h_{1 / 2}$, cf. 38.

(5) Let $\alpha \in(1,2)$ and $Y^{D}$ be a censored $\alpha$-stable process in $D$. Then it follows from [10] that $\mathbf{H K}_{\mathbf{B}}^{\mathbf{h}}$ is satisfied with $\Phi(r)=\Psi(r)=r^{\alpha}$ and boundary function $h_{(\alpha-1) / \alpha}$.

(6) Let $\alpha \in(0,2)$ and $Z^{D}$ be the part process, in $D$, of a reflected isotropic $\alpha$-stable process in $\bar{D}$. For any $q \in[\alpha-1, \alpha) \cap(0, \alpha)$, let $Y^{D}$ be the process on $D$ corresponding to the Feynman-Kac semigroup of $Z^{D}$ via the multiplicative functional $\exp \left(-\int_{0}^{t} C(d, \alpha, q) \operatorname{dist}\left(Z_{s}^{D}, \partial D\right)^{-\alpha} d s\right)$, where the positive constant $C(d, \alpha, q)$ is defined on [25, p. 233]. It follows from [25, Theorem 3.2] that the small time estimates (3.11) holds with $\Phi(r)=\Psi(r)=r^{\alpha}$ and $h_{q / \alpha}$. Using the small time estimates and the argument in [20, Section 4], one can easily show that the semigroup of $Y^{D}$ is intrinsically ultracontractive. With this, one can easily check that the large time estimates in Definition 3.6(a)(ii) holds. Thus $\mathbf{H K}_{\mathbf{B}}^{\mathbf{h}}$ holds.

(7) Suppose that $D$ is connected, $d \geq 3$ and $\kappa \geq-\frac{1}{4}$. Let $Y^{D}$ be the process corresponding to $\left.\Delta\right|_{D}-\kappa \delta_{D}(x)^{-2}$, the Dirichlet Laplacian in $D$ with critical potential $\kappa \delta_{D}(x)^{-2}$. It follows from [28, (6)] and [29, Corollary 1.8] that the heat kernel of $Y^{D}$ satisfies $\mathbf{H K}_{\mathbf{B}}^{\mathbf{h}}$ with $C_{0}=0, \Phi(r)=r^{2}$ and boundary function $h_{p}$, where $p=\frac{1}{2}\left(\frac{1}{2}+\sqrt{\frac{1}{4}+\kappa}\right)$.

(8) Suppose that $\alpha \in(1,2)$ and $d \geq 2$. Let $b: \mathbb{R}^{d} \rightarrow \mathbb{R}^{d}$ such that $|b|$ is in the Kato class $\mathbb{K}_{d, \alpha-1}$ (see [12, Definition 1.1] for definition). Let $Y$ be an $\alpha$-stable process with drift $b$ in $\mathbb{R}^{d}$, that is, a process with generator $-(-\Delta)^{\alpha / 2}+b \cdot \nabla$, and let $Y^{D}$ be the part process of $Y$ in $D$. By [12, Theorem 1.3], $\mathbf{H K}_{\mathbf{B}}^{\mathbf{h}}$ holds with $\Phi(r)=\Psi(r)=r^{\alpha}$ and $h_{1 / 2}$. See also 39].

(9) For general setups in which $\mathbf{H K}_{\mathbf{B}}^{\mathbf{h}}$ is satisfied, see [25, Section 2] and [30].

(b) Suppose that $D$ is an unbounded $C^{1,1}$ open subset of $\mathbb{R}^{d}$.

(1) If $D$ is the domain above the graph of a bounded Lipschitz function in $\mathbb{R}^{d-1}$, then the killed Brownian motion in $D$ satisfies $\mathbf{H K}_{\mathbf{U}}^{\mathbf{h}}$ with $C_{0}=0, \Phi(r)=r^{2}$ and a boundary function defined in terms of survival probabilities like in Example 3.5(b), which is of Harnack type (cf. [51]).

(2) Suppose that $D$ is a half-space-like $C^{1,1}$ open set in $\mathbb{R}^{d}$ and $\alpha \in(0,2)$. Let $Y^{D}$ be the part process in $D$ of an isotropic $\alpha$-stable process. Then by [21, Theorem 1.2], $\mathbf{H K}_{\mathbf{U}}^{\mathbf{h}}$ is satisfied with $\Phi(r)=\Psi(r)=r^{\alpha}$ and boundary function $h_{1 / 2}$. More generally, let $Y^{D}$ be the part process in $D$ of the independent sum of Brownian motion and an isotropic $\alpha$-stable process. By [13, Theorem 1.4 and Remark 1.5(ii)], $\mathbf{H K}_{\mathbf{U}}^{\mathbf{h}}$ is 
satisfied with $\Phi(r)=r^{2} \wedge r^{\alpha}, \Psi(r)=r^{\alpha}$ and boundary function $h_{1 / 2}$. When $D$ is an exterior $C^{1,1}$ open set in $\mathbb{R}^{d}$ with $d>\alpha$ and $Y^{D}$ is part process in $D$ of an isotropic $\alpha$-stable process, it follows from 21, Theorem 1.2] that $\mathbf{H K}_{\mathbf{U}}^{\mathbf{h}}$ is satisfied with $\Phi(r)=\Psi(r)=r^{\alpha}$ and boundary function $h_{1 / 2}(t \wedge 1, x, y)$. See 35. for a more general example.

(3) Suppose $D$ is the upper half space in $\mathbb{R}^{d}$. Let $\chi$ be a complete Bernstein function satisfying global weak scaling conditions with indices $\alpha_{1}, \alpha_{2} \in(0,1), Y$ be a subordinate Brownian motion in $\mathbb{R}^{d}$ via an independent subordinator with Laplace exponent $\chi, Y^{D}$ be the part process of $Y$ in $D$. It follows from [40, Theorem 5.10] that $\mathbf{H K}_{\mathbf{U}}^{\mathbf{h}}$ is satisfied with $\Phi(r)=\Psi(r)=1 / \chi\left(r^{-2}\right)$ and boundary function $h_{1 / 2}$. See [7] for a more general example.

(4) Suppose that $D$ is the upper half space in $\mathbb{R}^{d}$ and $\alpha \in(0,2)$. Let $Z^{D}$ be the part process, in $D$, of a reflected isotropic $\alpha$-stable process in $\bar{D}$. For any $q \in[\alpha-1, \alpha) \cap(0, \alpha)$, let $Y^{D}$ be the process on $D$ corresponding to the Feynman-Kac semigroup of $Z^{D}$ via the multiplicative functional $\exp \left(-\int_{0}^{t} C(d, \alpha, q) \delta_{D}\left(Z_{s}^{D}\right)^{-\alpha} d s\right)$, where $C(d, \alpha, q)$ is defined on [25, p. 233]. It follows from [25, Theorem 3.2 ] that $\mathbf{H K}_{\mathbf{U}}^{\mathbf{h}}$ is satisfied with $\Phi(r)=\Psi(r)=r^{\alpha}$ and boundary function $h_{q / \alpha}$.

(5) Suppose that $D=\mathbb{R}^{d} \backslash\{0\}$ and $\alpha \in(0,2)$. Let $Z$ be an isotropic $\alpha$-stable process in $\mathbb{R}^{d}$. For any $q \in(0, \alpha)$, let $Y^{D}$ be the process on $D$ corresponding to the Feynman-Kac semigroup of $Z^{D}$ via the multiplicative functional $\exp \left(-\int_{0}^{t} \widetilde{C}(d, \alpha, q)\left|Z_{s}^{D}\right|^{-\alpha} d s\right)$, where $\widetilde{C}(d, \alpha, q)$ is defined on [25, p. 250]. It follows from [25, Theorem 3.9] and [33, Theorem 1.1] that $\mathbf{H K}_{\mathbf{U}}^{\mathbf{h}}$ is satisfied with $\Phi(r)=\Psi(r)=r^{\alpha}$ and boundary function $h_{q / \alpha}$.

(6) Suppose that $D=\mathbb{R}^{d} \backslash\{0\}, d \geq 2$ or $D=(0, \infty)$. Let $Y^{D}$ be a process with generator $\Delta+(a-$ 1) $|x|^{-2} \sum_{i, j=1}^{d} x_{i} x_{j} \partial_{i j}+\kappa|x|^{-2} \cdot \nabla-b|x|^{-2}$ for some $a>0, \kappa, b \in \mathbb{R}$ such that

$$
\Lambda:=\frac{1}{2} \sqrt{\frac{b}{a}+\left(\frac{d-1+\kappa-a}{2 a}\right)^{2}} \geq \frac{1}{4 a}((d-1+\kappa-a) \vee((2 a-1) d+1-\kappa-3 a)) .
$$

Note that when $a=1$ and $\kappa, b \geq 0$, the above inequality is always true. It follows from [46, Proposition 4.14, Theorem 6.2, Corollary 6.4] that $\mathbf{H} \mathbf{K}_{\mathbf{U}}^{\mathbf{h}}$ is satisfied with $C_{0}=0, \Phi(r)=r^{2}$ and boundary function $h_{p, q}$ where $p=\Lambda-(d-1+\kappa-a) /(4 a)$ and $q=\Lambda-((2 a-1) d+1-\kappa-3 a) /(4 a)$.

(7) Suppose that $\alpha \in(1,2)$ and $D=\mathbb{R}^{d} \backslash\{0\}, d \geq 3$. Let $Y^{D}$ be a process with generator $-(-\Delta)^{-\alpha / 2}+$ $\kappa|x|^{-\alpha} x \cdot \nabla$ for some $\kappa \in(0, \infty)$. It follows from [45, Theorems 4 and 5] that $\mathbf{H K}_{\mathbf{U}}^{\mathbf{h}}$ is satisfied with $\Phi(r)=\Psi(r)=r^{\alpha}$ and boundary function $h=h_{0, \beta / \alpha}$ for $\beta \in(0, \alpha)$ determined by the equation at the beginning of [45, Section 3.2].

We now briefly discuss the term

$$
I\left(t, x, y, C_{0}\right):=\frac{1}{V\left(x, \Phi^{-1}(t)\right)} \wedge\left(\frac{C_{0} t}{V(x, \rho(x, y)) \Psi(\rho(x, y))}+\frac{1}{V\left(x, \Phi^{-1}(t)\right)} \exp \left(-\frac{c_{1} \rho(x, y)^{2}}{\Phi^{-1}(t)^{2}}\right)\right)
$$

appearing in (3.11). If $C_{0}=0$, then clearly

$$
I(t, x, y, 0)=\frac{1}{V\left(x, \Phi^{-1}(t)\right)} \exp \left(-\frac{c_{1} \rho(x, y)^{2}}{\Phi^{-1}(t)^{2}}\right)
$$

Suppose now that $C_{0}=1$.

Lemma 3.10. For any $a \geq 1$, there are comparability constants depending on a such that

$$
I(t, x, y, 1) \asymp \begin{cases}\frac{1}{V\left(x, \Phi^{-1}(t)\right)}, & t \geq a^{-1} \Phi(\rho(x, y)), \\ \frac{t}{V(x, \rho(x, y)) \Psi(\rho(x, y))}+\frac{1}{V\left(x, \Phi^{-1}(t)\right)} \exp \left(-\frac{c \rho(x, y)^{2}}{\Phi^{-1}(t)^{2}}\right), & t<a \Phi(\rho(x, y)) .\end{cases}
$$

In particular, if $\Psi(r) \simeq \Phi(r)$ for $r \in\left(0, R_{1}\right)$, then

$$
I(t, x, y, 1) \simeq \frac{1}{V\left(x, \Phi^{-1}(t)\right)} \wedge \frac{t}{V(x, \rho(x, y)) \Phi(\rho(x, y))}, \quad t>0, x, y \in D, \rho(x, y)<R_{1} .
$$

Proof. If $t \geq a^{-1} \Phi(\rho(x, y))$, then by (3.5),

$$
\frac{1}{V\left(x, \Phi^{-1}(t)\right)} \geq I(t, x, y, 1) \geq \frac{1}{V\left(x, \Phi^{-1}(t)\right)} \exp \left(-\frac{c_{1} \rho(x, y)^{2}}{\Phi^{-1}(t)^{2}}\right) \geq \frac{c_{2}\left(a, c_{1}\right)}{V\left(x, \Phi^{-1}(t)\right)} .
$$


Assume that $t<a \Phi(\rho(x, y))$. Set

$$
g(t, x, y):=\frac{t}{V(x, \rho(x, y)) \Psi(\rho(x, y))}+\frac{1}{V\left(x, \Phi^{-1}(t)\right)} \exp \left(-\frac{c_{1} \rho(x, y)^{2}}{\Phi^{-1}(t)^{2}}\right) .
$$

Clearly, $I(t, x, y, 1) \leq g(t, x, y)$. Further, by using that $\Psi \geq \Phi$ and (3.5), we have

$$
g(t, x, y) \leq \frac{a}{V\left(x, \Phi^{-1}(t / a)\right)}+\frac{1}{V\left(x, \Phi^{-1}(t)\right)} \leq \frac{c_{3}(a)+1}{V\left(x, \Phi^{-1}(t)\right)} .
$$

Hence, $I(t, x, y, 1)=V\left(x, \Phi^{-1}(t)\right)^{-1} \wedge g(t, x, y) \geq\left(c_{3}(a)+1\right)^{-1} g(t, x, y)$. Thus, (3.15) holds.

Now, we assume that $\Psi(r) \simeq \Phi(r)$ for $r \in\left(0, R_{1}\right)$. Using (3.1), (3.5) and the fact that $e^{-u} \leq k^{k} u^{-k}$ for all $u, k>0$, we get that for all $t>0$ and $x, y \in D$ satisfying $t<\Phi(\rho(x, y))$ and $\rho(x, y)<R_{1}$,

$$
\begin{aligned}
\frac{1}{V\left(x, \Phi^{-1}(t)\right)} \exp \left(-\frac{c_{1} \rho(x, y)^{2}}{\Phi^{-1}(t)^{2}}\right) & \leq \frac{c_{4}}{V\left(x, \Phi^{-1}(t)\right)}\left(\frac{\Phi^{-1}(t)^{2}}{c_{1} \rho(x, y)^{2}}\right)^{\left(d_{2}+\alpha_{1}\right) / 2} \\
& \leq \frac{c_{5} t}{V(x, \rho(x, y)) \Phi(\rho(x, y))} \leq \frac{c_{6} t}{V(x, \rho(x, y)) \Psi(\rho(x, y))} .
\end{aligned}
$$

Thus, we can deduce (3.16) from (3.15).

\section{JUMP KERNEL AND HEAT KERNEL ESTIMATES}

For a given boundary function $h$, we define for $(t, x, y) \in[0, \infty) \times D \times D$,

$$
\mathcal{B}_{h}^{*}(x, y):=\int_{0}^{\Phi(\rho(x, y))} h(s, x, y) w(s) d s
$$

and if $\phi^{-1}(1 / t)^{-1} \leq \Phi(\rho(x, y))$,

$$
\mathcal{B}_{h}(t, x, y):=\int_{2 \phi^{-1}(1 / t)^{-1}}^{4 \Phi(\rho(x, y))} h(s, x, y) w(s) d s .
$$

Since $\int_{0}^{r} w(s) d s<\infty$ for all $r>0$ (see (2.1)) and $h \leq 1$, the integral in (4.1) converges. Note that, by $(\mathrm{H} 1), \mathcal{B}_{h}^{*}(x, y) \simeq \mathcal{B}_{h}(0, x, y)$ for all $(x, y) \in D \times D$.

4.1. Jump kernel estimates. The jump kernel of the subordinate process $X$ is given by

$$
J(x, y)=\int_{0}^{\infty} p_{D}(s, x, y) \nu(d s), \quad x, y \in D .
$$

See [6, p.74] and also [48].

Theorem 4.1. Suppose that either (1) (Poly- $\left.R_{1}\right)$ and $\mathbf{H} \mathbf{K}_{\mathbf{B}}^{\mathbf{h}}$ hold, or (2) (Poly- $\infty$ ) and $\mathbf{H} \mathbf{K}_{\mathbf{U}}^{\mathbf{h}}$ hold. Then, for $(x, y) \in D \times D$ with $x \neq y$,

$$
J(x, y) \simeq \frac{C_{0} \mathcal{B}_{h}^{*}(x, y)}{V(x, \rho(x, y)) \Psi(\rho(x, y))}+h(\Phi(\rho(x, y)), x, y) \frac{w(\Phi(\rho(x, y)))}{V(x, \rho(x, y))} .
$$

Proof. Since the proofs are similar, we only give the proof of the case (1), which is more complicated. Fix $x, y \in D$ with $x \neq y$ and let $r:=\rho(x, y)>0$. By Remark 3.7, (3.11) and (3.12) hold with $T:=\Phi(2 \operatorname{diam}(D))$. Then by (4.3) and (3.15),

$$
\begin{aligned}
J(x, y) \asymp & \frac{C_{0}}{V(x, r) \Psi(r)} \int_{0}^{\Phi(r)} \operatorname{sh}(s, x, y) \nu(d s)+\int_{0}^{\Phi(r)} \frac{h(s, x, y)}{V\left(x, \Phi^{-1}(s)\right)} \exp \left(-\frac{c r^{2}}{\Phi^{-1}(s)^{2}}\right) \nu(d s) \\
& +\int_{\Phi(r)}^{T} \frac{h(s, x, y)}{V\left(x, \Phi^{-1}(s)\right)} \nu(d s)+h(1, x, y) \int_{T}^{\infty} e^{-\lambda_{D} s} \nu(d s)=: C_{0} J_{1}+J_{2}+J_{3}+J_{4} .
\end{aligned}
$$

Since (Poly- $\left.R_{1}\right)$ holds, there exists a constant $a>1$ such that $w(s / a) \geq 2 w(s)$ for all $s<R_{1}$. Therefore, by (3.10), since we assumed $\Phi(\operatorname{diam}(D))<R_{1} / 8$,

$$
V(x, r) \Psi(r) J_{1}=\sum_{i \in \mathbb{N}} \int_{a^{-i} \Phi(r)}^{a^{-i+1} \Phi(r)} \operatorname{sh}(s, x, y) \nu(d s)
$$




$$
\begin{aligned}
& \simeq \sum_{i \in \mathbb{N}} a^{-i} \Phi(r) h\left(a^{-i} \Phi(r), x, y\right)\left(w\left(a^{-i} \Phi(r)\right)-w\left(a^{-i+1} \Phi(r)\right)\right) \\
& \simeq \sum_{i \in \mathbb{N}} a^{-i} \Phi(r) h\left(a^{-i} \Phi(r), x, y\right) w\left(a^{-i} \Phi(r)\right) \simeq \sum_{i \in \mathbb{N}} \int_{a^{-i} \Phi(r)}^{a^{-i+1} \Phi(r)} h(s, x, y) w(s) d s=\mathcal{B}_{h}^{*}(x, y) .
\end{aligned}
$$

Next, by (H1), the scaling and monotonicity of $\Phi$, we get that

$$
\begin{aligned}
J_{2} & \geq \frac{h(\Phi(r), x, y)}{V(x, r)} \int_{\Phi(r) / a}^{\Phi(r)} \exp \left(-\frac{c_{1} r^{2}}{\Phi^{-1}(s)^{2}}\right) \nu(d s) \geq \frac{c_{2} h(\Phi(r), x, y)}{V(x, r)} \int_{\Phi(r) / a}^{\Phi(r)} \nu(d s) \\
& =\frac{c_{2} h(\Phi(r), x, y)}{V(x, r)}(w(\Phi(r) / a)-w(\Phi(r))) \geq \frac{c_{2} h(\Phi(r), x, y) w(\Phi(r))}{V(x, r)} .
\end{aligned}
$$

Hence, we obtain the lower bound in (4.4).

Now, we prove the upper bound in (4.4). Let $\widetilde{\Phi}$ be the function in Lemma 3.1. Since $s \mapsto V\left(x, \widetilde{\Phi}^{-1}(s)\right)^{-1}$ and $s \mapsto h(s, x, y)$ are non-increasing, using the Leibniz rule for product, integration by parts and the property (P2) of $\widetilde{\Phi}^{-1}$ in Lemma 3.1, we obtain

$$
\begin{aligned}
J_{2} & \leq c \int_{0}^{\Phi(r)} \frac{h(s, x, y)}{V\left(x, \widetilde{\Phi}^{-1}(s)\right)} \exp \left(-\frac{c_{3} r^{2}}{\widetilde{\Phi}^{-1}(s)^{2}}\right)\left(-\frac{d}{d s} w(s)\right) \\
& \leq c \int_{0}^{\Phi(r)} \frac{h(s, x, y) w(s)}{V\left(x, \widetilde{\Phi}^{-1}(s)\right)}\left(\frac{d}{d s} \exp \left(-\frac{c_{3} r^{2}}{\widetilde{\Phi}^{-1}(s)^{2}}\right)\right) d s \\
& \leq c \int_{0}^{\Phi(r)} \frac{h(s, x, y) w(s)}{V\left(x, \widetilde{\Phi}^{-1}(s)\right)} \frac{r^{2}}{s \widetilde{\Phi}^{-1}(s)^{2}} \exp \left(-\frac{c_{3} r^{2}}{\widetilde{\Phi}^{-1}(s)^{2}}\right) d s .
\end{aligned}
$$

In the second inequality above, we used the following: Since $h \leq 1, e^{-x} \leq k^{k} x^{-k}$ for all $x, k>0$ and $\lim _{s \rightarrow 0} s w(s)=0$ (because $w$ is the tail of the Lévy mesure $\nu$ ), by using (3.1) and the scaling of $\widetilde{\Phi}^{-1}$, we have that

$$
\begin{aligned}
& \lim _{s \rightarrow 0} \frac{h(s, x, y) w(s)}{V\left(x, \widetilde{\Phi}^{-1}(s)\right)} \exp \left(-\frac{c_{3} r^{2}}{\widetilde{\Phi}^{-1}(s)^{2}}\right) \leq c \lim _{s \rightarrow 0} \frac{w(s)}{V\left(x, \widetilde{\Phi}^{-1}(s)\right)}\left(\frac{\widetilde{\Phi}^{-1}(s)^{2}}{r^{2}}\right)^{\left(d_{2}+\alpha_{2}\right) / 2} \\
& \leq \frac{c}{r^{d_{2}+\alpha_{2}} V\left(x, \widetilde{\Phi}^{-1}(1)\right)} \lim _{s \rightarrow 0} w(s) \widetilde{\Phi}^{-1}(s)^{\alpha_{2}} \leq \frac{c \widetilde{\Phi}^{-1}(1)^{\alpha_{2}}}{r^{d_{2}+\alpha_{2}} V\left(x, \widetilde{\Phi}^{-1}(1)\right)} \lim _{s \rightarrow 0} s w(s)=0 .
\end{aligned}
$$

By $\left(\right.$ Poly- $\left.R_{1}\right)$, (H2), (3.1), (3.5) and the fact that $\Phi \simeq \widetilde{\Phi}$, we can use Lemma 3.2 with $f(s)=$ $\left.h(s, x, y) w(s) V\left(x, \widetilde{\Phi}^{-1}(s)\right)^{-1} s^{-1} \widetilde{\Phi}^{-1}(s)\right)^{-2}$ and $p=\gamma+\beta_{2}+1+\left(d_{2}+2\right) / \alpha_{1}$ to deduce from (4.5) that

$$
J_{2} \leq c \frac{\Phi(r)^{\gamma+\beta_{2}+1+\left(d_{2}+2\right) / \alpha_{1}+1}}{\Phi(r)^{\gamma+\beta_{2}+1+\left(d_{2}+2\right) / \alpha_{1}}} \frac{h(\Phi(r), x, y) w(\Phi(r)) r^{2}}{V(x, r) \Phi(r) r^{2}}=\frac{c h(\Phi(r), x, y) w(\Phi(r))}{V(x, r)} .
$$

For $J_{3}$ and $J_{4}$, since $s \mapsto V\left(x, \Phi^{-1}(s)\right)^{-1}, s \mapsto h(s, x, y)$ and $s \mapsto w(s)$ are non-increasing, we have by the boundedness of $D$ that

$$
J_{3}+J_{4} \leq \frac{h(\Phi(r), x, y) w(\Phi(r))}{V(x, r)}+h(1, x, y) w(T) \leq \frac{\operatorname{ch}(\Phi(r), x, y) w(\Phi(r))}{V(x, r)} .
$$

This completes the proof.

Suppose that $\Psi \simeq \Phi$ and $C_{0}=1$. Then the first term in (4.4) dominates the second. Indeed, by (H1),

$$
\begin{aligned}
\mathcal{B}_{h}^{*}(x, y) & =\int_{0}^{\Phi(\rho(x, y))} h(s, x, y) w(s) d s \geq h(\Phi(\rho(x, y)), x, y) \int_{0}^{\Phi(\rho(x, y))} w(s) d s \\
& \geq h(\Phi(\rho(x, y)), x, y) w(\Phi(\rho(x, y))) \Phi(\rho(x, y)) .
\end{aligned}
$$

Moreover, if $\beta_{2}<1$, then according to [47, Lemma 2.6, Proposition 2.9] and (2.2), we get that $w(s) \simeq$ $\phi(1 / s)$ for all $0<s<R_{1} / 2$. Therefore, it holds that

$$
J(x, y) \simeq \frac{1}{V(x, \rho(x, y)) \Phi(\rho(x, y))} \int_{0}^{\Phi(\rho(x, y))} h(s, x, y) \phi(1 / s) d s .
$$


In case the boundary function is equal to $h_{1 / 2}$, the integral above can be estimated in the same way as in [42, Lemma 8.1], cf. [42, (8.4)].

Suppose that $C_{0}=0$. Then

$$
J(x, y) \simeq h\left(\Phi(\rho(x, y)) \frac{w(h(\Phi(\rho(x, y)), x, y)}{V(x, \rho(x, y))} .\right.
$$

In particular, in the context of Example 3.9(b-1), and assuming $\beta_{2}<1$, the above formula reduces to [41, Theorem 4.4.(1)]. Similarly, if $D$ is an exterior $C^{1,1}$ domain in $\mathbb{R}^{d}$, the boundary function is equal to $h_{1 / 2}(t \wedge 1, x, y)$ and $\beta_{2}<1$, then (4.7) reduces to [41, Theorem 4.4.(2)].

\subsection{Heat kernel estimates. Let}

$$
\psi(r):=\frac{1}{\phi(1 / \Phi(r))}, \quad r>0 .
$$

Since $\phi$ and $\Phi$ are strictly increasing, $\psi$ is also strictly increasing. Moreover, it follows from (2.2), (2.3) and (3.4) that, for every $R_{0}>0$, there exist $c_{1}, c_{2}>0$ such that

$$
c_{1}\left(\frac{R}{r}\right)^{\alpha_{1} \beta_{1}} \leq \frac{\psi(R)}{\psi(r)} \leq c_{2}\left(\frac{R}{r}\right)^{\alpha_{2}\left(\beta_{2} \wedge 1\right)}, \quad 0<r<R<R_{0} .
$$

In case when (Poly- $\infty$ ) holds, (4.9) is valid with $R_{0}=\infty$. We note that

$$
\psi^{-1}(t)=\Phi^{-1}\left(\phi^{-1}(1 / t)^{-1}\right), \quad t>0 .
$$

Recall the definition of the function $\mathcal{B}_{h}(t, x, y)$ from (4.2).

Theorem 4.2. Suppose that $\left(\mathbf{P o l y}-R_{1}\right)$ and $\mathbf{H K}_{\mathbf{B}}^{\mathbf{h}}$ hold. Then for every $T>0$, the following estimates are valid for all $(t, x, y) \in(0, T] \times D \times D$ :

(i) If $\psi(\rho(x, y)) \leq t$, then

$$
q(t, x, y) \simeq \frac{h\left(\phi^{-1}(1 / t)^{-1}, x, y\right)}{V\left(x, \psi^{-1}(t)\right)}
$$

(ii) If $\psi(\rho(x, y)) \geq t$, then

$$
\begin{aligned}
q(t, x, y) \asymp & \frac{C_{0}}{V(x, \rho(x, y)) \Psi(\rho(x, y))}\left(t \mathcal{B}_{h}(t, x, y)+\frac{h\left(\phi^{-1}(1 / t)^{-1}, x, y\right)}{\phi^{-1}(1 / t)}\right) \\
& +\frac{h\left(\phi^{-1}(1 / t)^{-1}, x, y\right)}{V\left(x, \psi^{-1}(t)\right)} \exp \left(-\frac{c \rho(x, y)^{2}}{\psi^{-1}(t)^{2}}\right)+h(\Phi(\rho(x, y)), x, y) \frac{t w(\Phi(\rho(x, y)))}{V(x, \rho(x, y))} .
\end{aligned}
$$

Proof. Take $x, y \in D$ and let $r:=\rho(x, y)$. We start by establishing some relations valid for all $t \in(0, T]$. By Proposition 2.7, there exist constants $\delta, \epsilon \in(0,1)$ such that

$$
q(t, x, y) \geq \delta \inf _{s \in\left[\epsilon \phi^{-1}(1 / t)^{-1}, \phi^{-1}(1 / t)^{-1}\right]} p_{D}(s, x, y), \quad t \in(0, T] .
$$

On the other hand, by Remark 3.7 (with $T=\Phi(\operatorname{diam}(D)))$, (3.14), (3.15), (3.12) and the fact that $\exp \left(-c r^{2} / \Phi^{-1}(s)^{2}\right) \simeq 1$ when $s>\Phi(r)$, we see that

$$
\begin{aligned}
& q(t, x, y) \\
& \asymp \int_{0}^{\Phi(r)} h(s, x, y)\left(\frac{C_{0} s}{V(x, r) \Psi(r)}+\frac{1}{V\left(x, \Phi^{-1}(s)\right)} \exp \left(-\frac{c r^{2}}{\Phi^{-1}(s)^{2}}\right)\right) \mathbb{P}\left(S_{t} \in d s\right) \\
& \quad+\int_{\Phi(r)}^{\Phi(\operatorname{diam}(D))} \frac{h(s, x, y)}{V\left(x, \Phi^{-1}(s)\right)} \mathbb{P}\left(S_{t} \in d s\right)+h(1, x, y) \int_{\Phi(\operatorname{diam}(D))}^{\infty} e^{-\lambda_{D} s} \mathbb{P}\left(S_{t} \in d s\right) \\
& \asymp C_{0} \int_{0}^{\Phi(r)} \frac{s h(s, x, y)}{V(x, r) \Psi(r)} \mathbb{P}\left(S_{t} \in d s\right)+\int_{0}^{\Phi(\operatorname{diam}(D))} \frac{h(s, x, y)}{V\left(x, \Phi^{-1}(s)\right)} \exp \left(-\frac{c r^{2}}{\Phi^{-1}(s)^{2}}\right) \mathbb{P}\left(S_{t} \in d s\right) \\
& \quad+h(1, x, y) \int_{\Phi(\operatorname{diam}(D))}^{\infty} e^{-\lambda_{D} s} \mathbb{P}\left(S_{t} \in d s\right)=: C_{0} I_{1}+I_{2}+I_{3} .
\end{aligned}
$$


(i) Assume that $\psi(r) \leq t$. By Remark 3.7, (3.15), (H1), (3.1), the scaling of $\Phi^{-1}$ and (4.10), there exists a constant $c_{1}>0$ such that

$$
\inf _{s \in\left[\epsilon \phi^{-1}(1 / t)^{-1}, \phi^{-1}(1 / t)^{-1}\right]} p_{D}(s, x, y) \geq c_{1} \frac{h\left(\phi^{-1}(1 / t)^{-1}, x, y\right)}{V\left(x, \psi^{-1}(t)\right)}, \quad t \in(0, T] .
$$

Hence, the lower bound in (4.11) follows from (4.13).

Now, we prove the upper bound in (4.11). First, using Lemma 2.6 in the first inequality below, the assumption $\Psi \geq \Phi$ and Lemma 2.5 with $N=\gamma+d_{2} / \alpha_{1}$ in the second, (H2), (4.10) and (3.4) in the third, and (3.1) in the last, we get that

$$
\begin{aligned}
I_{1} & \leq \frac{\Phi(r) h(\Phi(r), x, y)}{V(x, r) \Psi(r)} \exp \left(-\frac{t}{2}(H \circ \sigma)(t, \Phi(r))\right) \\
& \leq c_{2} \frac{h(\Phi(r), x, y)}{V(x, r)}\left(\Phi(r) \phi^{-1}(1 / t)\right)^{\gamma}\left(\Phi(r) \phi^{-1}(1 / t)\right)^{d_{2} / \alpha_{1}} \\
& \leq c_{3} \frac{h\left(\phi^{-1}(1 / t)^{-1}, x, y\right)}{V(x, r)}\left(\frac{r}{\psi^{-1}(t)}\right)^{d_{2}} \leq c_{4} \frac{h\left(\phi^{-1}(1 / t)^{-1}, x, y\right)}{V\left(x, \psi^{-1}(t)\right)} .
\end{aligned}
$$

Next, we observe that

$$
I_{2} \leq \int_{0}^{\phi^{-1}(1 / t)^{-1}} \frac{h(s, x, y)}{V\left(x, \Phi^{-1}(s)\right)} \mathbb{P}\left(S_{t} \in d s\right)+\int_{\phi^{-1}(1 / t)^{-1}}^{\infty} \frac{h(s, x, y)}{V\left(x, \Phi^{-1}(s)\right)} \mathbb{P}\left(S_{t} \in d s\right)=: I_{2,1}+I_{2,2} .
$$

By (H2), (3.1) and (3.4), we can apply Lemma 2.6 with $f(s)=h(s, x, y) V\left(x, \Phi^{-1}(s)\right)^{-1}$ and $p=\gamma+d_{2} / \alpha_{1}$ to get that

$$
I_{2,1} \leq c_{5} \frac{h\left(\phi^{-1}(1 / t)^{-1}, x, y\right)}{V\left(x, \psi^{-1}(t)\right)} \exp \left(-\frac{t}{2}(H \circ \sigma)\left(t, \phi^{-1}(1 / t)^{-1}\right)\right) \leq c_{5} \frac{h\left(\phi^{-1}(1 / t)^{-1}, x, y\right)}{V\left(x, \psi^{-1}(t)\right)} .
$$

Moreover, we see from (H1), (4.10) and the monotonicity of $\psi^{-1}$ that

$$
I_{2,2} \leq \frac{h\left(\phi^{-1}(1 / t)^{-1}, x, y\right)}{V\left(x, \psi^{-1}(t)\right)} \mathbb{P}\left(S_{t} \geq \phi^{-1}(1 / t)^{-1}\right) \leq \frac{h\left(\phi^{-1}(1 / t)^{-1}, x, y\right)}{V\left(x, \psi^{-1}(t)\right)} .
$$

Lastly, by using (H1) and (H2), since $\phi$ and $\psi$ are increasing and $t \leq T$, we have that

$$
I_{3} \leq h(1, x, y) \leq c_{6} \frac{h\left(\phi^{-1}(1 / t)^{-1}, x, y\right)}{V\left(x, \psi^{-1}(t)\right)} .
$$

Hence, we obtain the upper bound in (4.11) from (4.14).

(ii) Assume that $\psi(r) \geq t$. First we establish the lower bound. From (4.13), Remark 3.7, (3.15), (4.10), (H1), and the scaling and monotonicity of $\psi^{-1}$, we get that

$$
q(t, x, y) \geq c_{7} h\left(\phi^{-1}(1 / t)^{-1}, x, y\right)\left[\frac{C_{0} \phi^{-1}(1 / t)^{-1}}{V(x, r) \Psi(r)}+\frac{1}{V\left(x, \psi^{-1}(t)\right)} \exp \left(-\frac{c_{8} r^{2}}{\psi^{-1}(t)^{2}}\right)\right] .
$$

We also see from Remark 3.7 that

$$
q(t, x, y) \geq \int_{2 \phi^{-1}(1 / t)^{-1}}^{4 \Phi(r)} p_{D}(s, x, y) \mathbb{P}\left(S_{t} \in d s\right) \geq \frac{c_{9} C_{0}}{V(x, r) \Psi(r)} \int_{2 \phi^{-1}(1 / t)^{-1}}^{4 \Phi(r)} \operatorname{sh}(s, x, y) \mathbb{P}\left(S_{t} \in d s\right),
$$

where in the second inequality we used (3.15) and neglected the second term. Let $M>1$ be the constant in Proposition 2.9. If $\Phi(r)>M \phi^{-1}(1 / t)^{-1}$, then by (H1), (H2) and Proposition 2.9, since we assumed $\Phi(\operatorname{diam}(D))<R_{1} / 8$,

$$
\begin{aligned}
& \int_{2 \phi^{-1}(1 / t)^{-1}}^{4 \Phi(r)} s h(s, x, y) \mathbb{P}\left(S_{t} \in d s\right) \geq \sum_{\substack{i \in \mathbb{N} \\
M^{i} \leq 2 \Phi(r) \phi^{-1}(1 / t)}} \int_{2 M^{i-1} \phi^{-1}(1 / t)^{-1}}^{2 M^{i} \phi^{-1}(1 / t)^{-1}} \operatorname{sh}(s, x, y) \mathbb{P}\left(S_{t} \in d s\right) \\
& \geq c_{10} M^{-1} t \sum_{\substack{i \in \mathbb{N} \\
M^{i} \leq 2 \Phi(r) \phi^{-1}(1 / t)}} 2 M^{i} \phi^{-1}(1 / t)^{-1} h\left(2 M^{i-1} \phi^{-1}(1 / t)^{-1}, x, y\right) w\left(2 M^{i-1} \phi^{-1}(1 / t)^{-1}\right) \\
& \geq c_{10} M^{-1} t \sum_{\substack{i \in \mathbb{N} \\
M^{i} \leq 2 \Phi(r) \phi^{-1}(1 / t)}} \int_{2 M^{i-1} \phi^{-1}(1 / t)^{-1}}^{2 M^{i} \phi^{-1}(1 / t)^{-1}} h(s, x, y) w(s) d s
\end{aligned}
$$




$$
\begin{aligned}
& \geq c_{10} M^{-1} t \int_{2 \phi^{-1}(1 / t)^{-1}}^{4 \Phi(r) / M} h(s, x, y) w(s) d s \geq c_{10} M^{-1} t \int_{2 \Phi(r) / M}^{4 \Phi(r) / M} h(s, x, y) w(s) d s \\
& \geq 2 c_{10} M^{-2} t \Phi(r) h(4 \Phi(r) / M, x, y) w(4 \Phi(r) / M) \geq c_{10} 2^{-1} M^{-2} t \int_{4 \Phi(r) / M}^{4 \Phi(r)} h(s, x, y) w(s) d s .
\end{aligned}
$$

By the fourth and the last inequalities above, we deduce from (4.16) that

$$
q(t, x, y) \geq \frac{c_{9} C_{0}}{2 V(x, r) \Psi(r)} 2 \int_{2 \phi^{-1}(1 / t)^{-1}}^{4 \Phi(r)} \operatorname{sh}(s, x, y) \mathbb{P}\left(S_{t} \in d s\right) \geq \frac{c_{9} c_{10}}{2 M^{2}} \frac{C_{0} t \mathcal{B}_{h}(t, x, y)}{V(x, r) \Psi(r)} .
$$

In case when $\Phi(r) \leq M \phi^{-1}(1 / t)^{-1}$, we see from (H1), (2.10) and (4.15) that

$$
\begin{aligned}
\frac{C_{0} t \mathcal{B}_{h}(t, x, y)}{V(x, r) \Psi(r)} & \leq \frac{C_{0} t}{V(x, r) \Psi(r)} \int_{2 \phi^{-1}(1 / t)^{-1}}^{4 M \phi^{-1}(1 / t)^{-1}} h(s, x, y) w(s) d s \\
& \leq \frac{4 e M C_{0}}{e-2} \frac{t \phi^{-1}(1 / t)^{-1}}{V(x, r) \Psi(r)} h\left(\phi^{-1}(1 / t)^{-1}, x, y\right) \phi\left(\phi^{-1}(1 / t)\right) \leq c_{11} q(t, x, y) .
\end{aligned}
$$

Hence, it remains to prove that there exists a constant $c_{12}>0$ such that

$$
q(t, x, y) \geq c_{12} h(\Phi(r), x, y) \frac{t w(\Phi(r))}{V(x, r)} .
$$

Recall that $M>1$ is the constant in Proposition 2.9, By using Proposition 2.9, (3.15), (H1), (3.1) and the scaling of $\Phi$, we get that, if $4 \Phi(r) / M>2 \phi^{-1}(1 / t)^{-1}$, then

$$
q(t, x, y) \geq c_{13} \int_{4 \Phi(r) / M}^{4 \Phi(r)} \frac{h(s, x, y)}{V\left(x, \Phi^{-1}(s)\right)} \mathbb{P}\left(S_{t} \in d s\right) \geq c_{14} h(\Phi(r), x, y) \frac{t w(\Phi(r))}{V(x, r)} .
$$

If $4 \Phi(r) / M \leq 2 \phi^{-1}(1 / t)^{-1}$, then by (4.10), the scaling of $\Phi$ and the assumption that $\psi(r) \geq t$, we get $\psi^{-1}(t) \leq r \leq c_{15} \psi^{-1}(t)$ for some $c_{15}>1$. By (4.10) and (2.10), since $w$ is non-increasing,

$$
t w(\Phi(r)) \leq t w\left(\phi^{-1}(1 / t)^{-1}\right) \leq e(e-2)^{-1} t \phi\left(\phi^{-1}(1 / t)\right)=e(e-2)^{-1} .
$$

Therefore, by (H1), (4.15) (neglecting the first term) and (3.4), we obtain

$$
q(t, x, y) \geq c_{16} \frac{h(\Phi(r), x, y)}{V(x, r)} \exp \left(-\frac{c_{8} c_{15} \psi^{-1}(t)^{2}}{\psi^{-1}(t)^{2}}\right) \geq c_{17} h(\Phi(r), x, y) \frac{t w(\Phi(r))}{V(x, r)} .
$$

This completes the proof of the lower bound.

Now we prove the upper bound. Recall (4.14). Observe that

$$
\begin{aligned}
V(x, r) \Psi(r) I_{1} \leq & \int_{0}^{\phi^{-1}(1 / t)^{-1}} \operatorname{sh}(s, x, y) \mathbb{P}\left(S_{t} \in d s\right)+\int_{\phi^{-1}(1 / t)^{-1}}^{2 \phi^{-1}(1 / t)^{-1}} \operatorname{sh}(s, x, y) \mathbb{P}\left(S_{t} \in d s\right) \\
& +\int_{2 \phi^{-1}(1 / t)^{-1}}^{4 \Phi(r)} \operatorname{sh}(s, x, y) \mathbb{P}\left(S_{t} \in d s\right)=: K_{1}+K_{2}+K_{3} .
\end{aligned}
$$

We get from Lemma 2.6 that $K_{1} \leq c_{18} \phi^{-1}(1 / t)^{-1} h\left(\phi^{-1}(1 / t)^{-1}, x, y\right)$. Next, by (H1), we have $K_{2} \leq$ $2 \phi^{-1}(1 / t)^{-1} h\left(\phi^{-1}(1 / t)^{-1}, x, y\right)$. To bound $K_{3}$, we use integration by parts and Proposition 2.9 to obtain

$$
\begin{aligned}
K_{3}= & \int_{2 \phi^{-1}(1 / t)^{-1}}^{4 \Phi(r)} \operatorname{sh}(s, x, y) \frac{d}{d s}\left(-\mathbb{P}\left(S_{t} \geq s\right)\right) \\
\leq & 2 \phi^{-1}(1 / t)^{-1} h\left(\phi^{-1}(1 / t)^{-1}, x, y\right)+\int_{2 \phi^{-1}(1 / t)^{-1}}^{4 \Phi(r)} h(s, x, y) \mathbb{P}\left(S_{t} \geq s\right) d s \\
& +\int_{2 \phi^{-1}(1 / t)^{-1}}^{4 \Phi(r)} s \mathbb{P}\left(S_{t} \geq s\right) \frac{d h(s, x, y)}{d s} \leq c_{19}\left(\phi^{-1}(1 / t)^{-1} h\left(\phi^{-1}(1 / t)^{-1}, x, y\right)+t \mathcal{B}_{h}(t, x, y)\right) .
\end{aligned}
$$

In the second inequality above, we used the fact that $s \mapsto h(s, x, y)$ is non-increasing (so that $s \mapsto$ $\frac{d}{d s} h(s, x, y) \leq 0$ a.e. $)$.

Now, we estimate $I_{2}$. We have

$$
I_{2} \leq \int_{0}^{2 \phi^{-1}(1 / t)^{-1}} \frac{h(s, x, y)}{V\left(x, \Phi^{-1}(s)\right)} \exp \left(-\frac{c r^{2}}{\Phi^{-1}(s)^{2}}\right) \mathbb{P}\left(S_{t} \in d s\right)
$$




$$
\begin{aligned}
& +\int_{2 \phi^{-1}(1 / t)^{-1}}^{4 \Phi(r)} \frac{h(s, x, y)}{V\left(x, \Phi^{-1}(s)\right)} \exp \left(-\frac{c r^{2}}{\Phi^{-1}(s)^{2}}\right) \mathbb{P}\left(S_{t} \in d s\right)+\int_{4 \Phi(r)}^{\infty} \frac{h(s, x, y)}{V\left(x, \Phi^{-1}(s)\right)} \mathbb{P}\left(S_{t} \in d s\right) \\
= & : L_{1}+L_{2}+L_{3} .
\end{aligned}
$$

By applying Lemma 2.6, we get from (H1), (H2), (3.1), the scaling of $\Phi$ and (4.10) that

$$
\begin{aligned}
L_{1} & \leq \exp \left(-\frac{c r^{2}}{\Phi^{-1}\left(2 \phi^{-1}(1 / t)^{-1}\right)^{2}}\right) \int_{0}^{2 \phi^{-1}(1 / t)^{-1}} \frac{h(s, x, y)}{V\left(x, \Phi^{-1}(s)\right)} \mathbb{P}\left(S_{t} \in d s\right) \\
& \leq c_{20} \frac{h\left(\phi^{-1}(1 / t)^{-1}, x, y\right)}{V\left(x, \psi^{-1}(t)\right)} \exp \left(-\frac{c_{21} r^{2}}{\psi^{-1}(t)^{2}}\right) .
\end{aligned}
$$

Let $\widetilde{\Phi}$ be the function in Lemma 3.1. By using integration by parts and similar calculations to (4.5), we get that

$$
\begin{aligned}
L_{2} \leq c_{22} & \int_{2 \phi^{-1}(1 / t)^{-1}}^{4 \Phi(r)} \frac{h(s, x, y)}{V\left(x, \widetilde{\Phi}^{-1}(s)\right)} \exp \left(-\frac{c_{23} r^{2}}{\widetilde{\Phi}^{-1}(s)^{2}}\right) \frac{d}{d s}\left(-\mathbb{P}\left(S_{t} \geq s\right)\right) \\
\leq c_{24} & {\left[\frac{h\left(2 \phi^{-1}(1 / t)^{-1}, x, y\right)}{V\left(x, \widetilde{\Phi}^{-1}\left(2 \phi^{-1}(1 / t)^{-1}\right)\right)} \exp \left(-\frac{c_{23} r^{2}}{\widetilde{\Phi}^{-1}\left(2 \phi^{-1}(1 / t)^{-1}\right)^{2}}\right) \mathbb{P}\left(S_{t} \geq 2 \phi^{-1}(1 / t)^{-1}\right)\right.} \\
& \left.+\int_{2 \phi^{-1}(1 / t)^{-1}}^{4 \Phi(r)} \frac{h(s, x, y)}{V\left(x, \widetilde{\Phi}^{-1}(s)\right)} \mathbb{P}\left(S_{t} \geq s\right) \frac{r^{2}}{s \widetilde{\Phi}^{-1}(s)^{2}} \exp \left(-\frac{c_{23} r^{2}}{\widetilde{\Phi}^{-1}(s)^{2}}\right) d s\right]=: c_{25}\left(L_{2,1}+L_{2,2}\right) .
\end{aligned}
$$

By (H1), (3.1), the scaling of $\Phi$ and (4.10), since $\Phi \simeq \widetilde{\Phi}$, we see that

$$
L_{2,1} \leq c_{26} h\left(\phi^{-1}(1 / t)^{-1}, x, y\right) V\left(x, \psi^{-1}(t)\right)^{-1} \exp \left(-c_{27} r^{2} / \psi^{-1}(t)^{2}\right) .
$$

Also, by using Proposition 2.9 and repeating the calculation in (4.6), we get that

$$
L_{2,2} \leq c_{28} t \int_{2 \phi^{-1}(1 / t)^{-1}}^{4 \Phi(r)} \frac{h(s, x, y) w(s)}{V\left(x, \widetilde{\Phi}^{-1}(s)\right)} \frac{r^{2}}{s \widetilde{\Phi}^{-1}(s)^{2}} \exp \left(-\frac{c_{23} r^{2}}{\widetilde{\Phi}^{-1}(s)^{2}}\right) d s \leq \frac{c_{29} t h(\Phi(r), x, y) w(\Phi(r))}{V(x, r)} .
$$

By (H1) and Proposition 2.9, we obtain

$$
L_{3} \leq h(\Phi(r), x, y) V(x, r)^{-1} \mathbb{P}\left(S_{t} \geq 4 \Phi(r)\right) \leq c_{30} t h(\Phi(r), x, y) V(x, r)^{-1} w(\Phi(r)) .
$$

Finally, we estimate $I_{3}$. By Proposition 2.9, since $D$ is bounded, we get from (H1) and (H2) that

$$
I_{3} \leq c_{31} t h(1, x, y) w(\Phi(\operatorname{diam}(D))) \leq c_{32} \operatorname{th}(\Phi(r), x, y) V(x, r)^{-1} w(\Phi(r)) .
$$

This completes the proof.

If one assumes $\mathbf{H K}_{\mathbf{U}}^{\mathbf{h}}$, instead of $\mathbf{H K}_{\mathbf{B}}^{\mathbf{h}}$, together with (Poly- $\infty$ ), the results of Theorem 4.2(i) and (ii) are valid for all time. The proof is analogous to the proof of Theorem 4.2 and hence omitted.

Theorem 4.3. Suppose that $(\mathbf{P o l y}-\infty)$ and $\mathbf{H K}_{\mathbf{U}}^{\mathbf{h}}$ hold. Then the assertions in Theorem 4.2(i)-(ii) hold for all $(t, x, y) \in(0, \infty) \times D \times D$.

When the upper scaling index $\beta_{2}$ in $\left(\mathbf{P o l y}-R_{1}\right)$ is strictly less than 1 , we can obtain the following simpler form of off-diagonal estimate.

Corollary 4.4. Suppose that (Poly- $\left.R_{1}\right)$ holds with $\beta_{2}<1$ and $\Phi(r) \simeq \Psi(r)$ for $r \in\left(0, R_{1}\right)$.

(i) If $\mathbf{H K}_{\mathbf{B}}^{\mathbf{h}}$ holds, then for every $T>0$, the following estimates hold for all $(t, x, y) \in(0, T] \times D \times D$ :

(1) If $\psi(\rho(x, y)) \leq t$, then (4.11) holds.

(2) If $\psi(\rho(x, y)) \geq t$, then

$$
q(t, x, y) \simeq \frac{t}{V(x, \rho(x, y))} \times \begin{cases}\frac{h(\Phi(\rho(x, y)), x, y)}{\psi(\rho(x, y))} & \text { when } C_{0}=0 \\ \frac{\mathcal{B}_{h}(t, x, y)}{\Phi(\rho(x, y))} & \text { when } C_{0}=1\end{cases}
$$

(ii) If $R_{1}=\infty$ and $\mathbf{H} \mathbf{K}_{\mathbf{U}}^{\mathbf{h}}$ holds, then (1) and (2) above hold for all $(t, x, y) \in(0, \infty) \times D \times D$. 
Proof. According to [47, Lemma 2.6, Proposition 2.9] and (2.2), since $\beta_{2}<1$, we get that $w(s) \simeq \phi(1 / s)$ for all $0<s<R_{1} / 2$.

(i) We only need to deal with the case (2), i.e., the case $\psi(\rho(x, y)) \geq t$. We first assume that $C_{0}=0$. Using $w(s) \simeq \phi(1 / s)$, we get that $w(\Phi(\rho(x, y))) \simeq 1 / \psi(\rho(x, y))$. Thus by Theorem 4.2, it remains to show that for any given $c_{1}>0$, there exists $c_{2}>0$ such that for all $(t, x, y) \in(0, T] \times D \times D$ satisfying $\psi(\rho(x, y)) \geq t$,

$$
\frac{h\left(\phi^{-1}(1 / t)^{-1}, x, y\right)}{V\left(x, \psi^{-1}(t)\right)} \exp \left(-\frac{c_{1} \rho(x, y)^{2}}{\psi^{-1}(t)^{2}}\right) \leq c_{2} \frac{t h(\Phi(\rho(x, y)), x, y)}{V(x, \rho(x, y)) \psi(\rho(x, y))} .
$$

Let $c_{3}:=\sup _{u>0} u^{\left(d_{2}+\alpha_{2} \gamma+\alpha_{2} \beta_{2}\right) / 2} e^{-u}$. By (3.1), (3.4), (4.9), (4.10) and (H2), we obtain that

$$
\begin{aligned}
& \frac{h\left(\phi^{-1}(1 / t)^{-1}, x, y\right)}{V\left(x, \psi^{-1}(t)\right)} \exp \left(-\frac{c_{1} \rho(x, y)^{2}}{\psi^{-1}(t)^{2}}\right) \leq c_{3} \frac{h\left(\phi^{-1}(1 / t)^{-1}, x, y\right)}{V\left(x, \psi^{-1}(t)\right)}\left(\frac{\psi^{-1}(t)^{2}}{c_{1} \rho(x, y)^{2}}\right)^{\left(d_{2}+\alpha_{2} \gamma+\alpha_{2} \beta_{2}\right) / 2} \\
& \leq c_{4} \frac{h\left(\phi^{-1}(1 / t)^{-1}, x, y\right)}{V(x, \rho(x, y))}\left(\frac{\Phi\left(\psi^{-1}(t)\right)}{\Phi(\rho(x, y))}\right)^{\gamma} \frac{t}{\psi(\rho(x, y))} \leq c_{5} \frac{t h(\Phi(\rho(x, y)), x, y)}{V(x, \rho(x, y)) \psi(\rho(x, y))} .
\end{aligned}
$$

Now, let $C_{0}=1$. Since $\Phi(r) \simeq \Psi(r)$ for $r \in\left(0, R_{1}\right)$, the first term on the right hand side of (4.12) is comparable with

$$
\frac{t \mathcal{B}_{h}(t, x, y)}{V(x, \rho(x, y)) \Phi(\rho(x, y))}+\frac{h\left(\phi^{-1}(1 / t)^{-1}, x, y\right)}{V(x, \rho(x, y)) \Phi(\rho(x, y)) \phi^{-1}(1 / t)}
$$

By (H2) and (Poly- $\left.R_{1}\right)$, it holds that for all $(t, x, y) \in(0, T] \times D \times D$ satisfying $\psi(\rho(x, y)) \geq t$,

$$
t \mathcal{B}_{h}(t, x, y) \geq t \int_{2 \Phi(\rho(x, y))}^{4 \Phi(\rho(x, y))} h(s, x, y) w(s) d s \geq c_{6} \operatorname{th}(\Phi(\rho(x, y)), x, y) w(\Phi(\rho(x, y))) \Phi(\rho(x, y)) .
$$

Combining this with (4.19), using $w(s) \simeq \phi(1 / s)$, one can see that the first term on the right hand side of (4.12) dominates the other two terms. Further, by (H2), (2.10) and (2.2), we see that for all $(t, x, y) \in(0, T] \times D \times D$ satisfying $\psi(\rho(x, y)) \geq t$,

$$
\begin{aligned}
t \mathcal{B}_{h}(t, x, y) & \geq t \int_{2 \phi^{-1}(1 / t)^{-1}}^{4 \phi^{-1}(1 / t)^{-1}} h(s, x, y) w(s) d s \geq c_{7} t \int_{2 \phi^{-1}(1 / t)^{-1}}^{4 \phi^{-1}(1 / t)^{-1}} h(s, x, y) \phi(1 / s) d s \\
& \geq c_{8} \phi^{-1}(1 / t)^{-1} h\left(\phi^{-1}(1 / t)^{-1}, x, y\right) .
\end{aligned}
$$

This yields the desired conclusion.

(ii) This can be proved by the same argument as that of (i). We omit the details here.

In the case when $D$ is a bounded $C^{1,1}$ domain, $Y^{D}$ is a killed Brownian motion in $D$ and $S$ is an $(\alpha / 2)$ stable subordinator, part (i) of the corollary above is equivalent to [49, Theorem 4.7]. In the case when $D$ is an exterior $C^{1,1}$ domain, $Y^{D}$ is a killed Brownian motion in $D$ and $S$ is an $(\alpha / 2)$-stable subordinator, part (ii) of the corollary above corrects [49, Theorem 4.6].

For future use, we note the following rough upper estimates on $q(t, x, y)$.

Proposition 4.5. (i) Suppose that $\left(\mathbf{P o l y}-R_{1}\right)$ and $\mathbf{H K}_{\mathbf{B}}^{\mathbf{h}}$ hold. Then for every $T>0$, there exists a constant $C>0$ such that for all $(t, x, y) \in(0, T] \times D \times D$,

$$
q(t, x, y) \leq C h\left(\phi^{-1}(1 / t)^{-1}, x, y\right)\left(\frac{1}{V\left(x, \psi^{-1}(t)\right)} \wedge \frac{t}{V(x, \rho(x, y)) \psi(\rho(x, y))}\right) .
$$

(ii) Suppose that $(\mathbf{P o l y}-\infty)$ and $\mathbf{H K}_{\mathbf{U}}^{\mathbf{h}}$ hold. Then, there exists a constant $C>0$ such that (4.20) holds for all $(t, x, y) \in(0, \infty) \times D \times D$.

Proof. (i) Take $x, y \in D$ and let $r:=\rho(x, y)$. If $\psi(r) \leq t$, then (4.20) follows from Theorem 4.2(i). Hence, we assume that $\psi(r) \geq t$ and estimate each term in Theorem 4.2(ii) separately.

First, by using (H1), the fact that $\Psi \geq \Phi$ and (2.1), we get

$$
\begin{aligned}
& \frac{t}{V(x, r) \Psi(r)} \int_{2 / \phi^{-1}(1 / t)}^{4 \Phi(r)} h(s, x, y) w(s) d s \leq \frac{t h\left(1 / \phi^{-1}(1 / t)^{-1}, x, y\right)}{V(x, r) \Phi(r)} \int_{0}^{4 \Phi(r)} w(s) d s \\
& \leq 4 e \frac{\operatorname{th}\left(1 / \phi^{-1}(1 / t)^{-1}, x, y\right)}{V(x, r)} \phi(1 /(4 \Phi(r))) \leq 4 e \frac{\operatorname{th}\left(\phi^{-1}(1 / t)^{-1}, x, y\right)}{V(x, r) \psi(r)} .
\end{aligned}
$$


Next, we note that, since $\phi$ is a Bernstein function, the map $u \mapsto \phi(u) / u$ is decreasing so that $\Phi(r) \phi(1 / \Phi(r)) \geq \phi^{-1}(1 / t)^{-1} \phi\left(\phi^{-1}(1 / t)\right)$. Hence, since $\Psi \geq \Phi$, it holds that

$$
\frac{h\left(\phi^{-1}(1 / t)^{-1}, x, y\right)}{V(x, r) \Psi(r) \phi^{-1}(1 / t)} \leq \frac{h\left(\phi^{-1}(1 / t)^{-1}, x, y\right)}{V(x, r) \psi(r) \phi\left(\phi^{-1}(1 / t)\right)}=\frac{t h\left(\phi^{-1}(1 / t)^{-1}, x, y\right)}{V(x, r) \psi(r)} .
$$

Thirdly, by (3.1) and (4.9), we see that for $c_{2}:=\sup _{u>0} u^{\left(d_{2}+\alpha_{2}\left(\beta_{2} \wedge 1\right)\right) / 2} e^{-u}$,

$$
\begin{aligned}
\frac{h\left(\phi^{-1}(1 / t)^{-1}, x, y\right)}{V\left(x, \psi^{-1}(t)\right)} \exp \left(-\frac{c_{1} r^{2}}{\psi^{-1}(t)^{2}}\right) & \leq c_{2} \frac{h\left(\phi^{-1}(1 / t)^{-1}, x, y\right)}{V\left(x, \psi^{-1}(t)\right)}\left(\frac{\psi^{-1}(t)^{2}}{c_{1} r^{2}}\right)^{\left(d_{2}+\alpha_{2}\left(\beta_{2} \wedge 1\right)\right) / 2} \\
& \leq c_{3} \frac{t h\left(\phi^{-1}(1 / t)^{-1}, x, y\right)}{V(x, r) \psi(r)}
\end{aligned}
$$

Lastly, by (H1) and (2.10),

$$
\frac{t h(\Phi(r), x, y) w(\Phi(r))}{V(x, r)} \leq \frac{e}{e-2} \frac{\operatorname{th}\left(\phi^{-1}(1 / t)^{-1}, x, y\right)}{V(x, r) \psi(r)} .
$$

(ii) By using Theorem 4.3 instead of Theorem 4.2, we obtain the result by repeating the proof of (i).

As a corollary to Theorems 4.2 and 4.3 , we obtain the following interior estimates on $q(t, x, y)$ in case of a regular boundary function.

Corollary 4.6. Suppose that $h(t, x, y)$ is a regular boundary function.

(i) If $\left(\mathbf{P o l y}-R_{1}\right)$ and $\mathbf{H K}_{\mathbf{B}}^{\mathbf{h}}$ hold, then for every $T>0$, the following estimates hold for all $(t, x, y) \in$ $(0, T] \times D \times D$ satisfying $\delta_{\wedge}(x, y) \geq \rho(x, y) \vee \psi^{-1}(t)$.

(1) If $\psi(\rho(x, y)) \leq t$, then $q(t, x, y) \simeq \frac{1}{V\left(x, \psi^{-1}(t)\right)}$.

(2) If $\psi(\rho(x, y)) \geq t$, then

$$
\begin{aligned}
q(t, x, y) \asymp & \frac{C_{0}}{V(x, \rho(x, y)) \Psi(\rho(x, y))}\left(t \int_{2 \phi^{-1}(1 / t)^{-1}}^{4 \Phi(\rho(x, y))} w(s) d s+\frac{1}{\phi^{-1}(1 / t)}\right) \\
& +\frac{1}{V\left(x, \psi^{-1}(t)\right)} \exp \left(-\frac{c \rho(x, y)^{2}}{\psi^{-1}(t)^{2}}\right)+\frac{t w(\Phi(\rho(x, y)))}{V(x, \rho(x, y))} .
\end{aligned}
$$

(ii) If (Poly- $\infty)$ and $\mathbf{H} \mathbf{K}_{\mathbf{U}}^{\mathbf{h}}$ hold, then (1) and (2) above hold for all $(t, x, y) \in(0, \infty) \times D \times D$ satisfying $\delta_{\wedge}(x, y) \geq \rho(x, y) \vee \psi^{-1}(t)$.

Now we give the large time estimates for $q(t, x, y)$ under $\mathbf{H K}_{\mathbf{B}}^{\mathbf{h}}$.

Theorem 4.7. Suppose that $\left(\mathbf{P o l y}-R_{1}\right)$ and $\mathbf{H K}_{\mathbf{B}}^{\mathbf{h}}$ hold. Then for every $T>0$,

$$
q(t, x, y) \simeq e^{-t \phi\left(\lambda_{D}\right)} h(1, x, y), \quad(t, x, y) \in[T, \infty) \times D \times D .
$$

Proof. Fix $x, y \in D$ and $s_{0} \in(0,1)$ such that $(H \circ \sigma)\left(T, s_{0}\right) \geq 2 \phi\left(\lambda_{D}\right)+1 / T$. Since $\lim _{s \rightarrow 0}(H \circ \sigma)(T, s)=$ $\infty$, such an $s_{0}$ always exists. Then, since $H$ is non-decreasing and $\phi^{\prime}$ is non-increasing, we see that

$$
(H \circ \sigma)\left(t, s_{0}\right) \geq 2 \phi\left(\lambda_{D}\right)+1 / T, \quad t \geq T .
$$

By (H2), (3.1) and (3.4), we can apply Lemma 2.6 with $f(s)=h(s, x, y) V\left(x, \Phi^{-1}(s)\right)^{-1}$. Using Remark 3.7 (with $T=s_{0}$ ), Lemma 2.6 and (4.22), since $\phi$ is the Laplace exponent of $S$, we get that, for all $t \geq T$,

$$
\begin{aligned}
q(t, x, y) & \leq c_{1} \int_{0}^{s_{0}} \frac{h(s, x, y)}{V\left(x, \Phi^{-1}(s)\right)} \mathbb{P}\left(S_{t} \in d s\right)+c_{1} h(1, x, y) \int_{s_{0}}^{\infty} e^{-\lambda_{D} s} \mathbb{P}\left(S_{t} \in d s\right) \\
& \leq c_{2} \frac{h\left(s_{0}, x, y\right)}{V\left(x, \Phi^{-1}\left(s_{0}\right)\right)} \exp \left(-\frac{t}{2}(H \circ \sigma)\left(t, s_{0}\right)\right)+c_{1} h(1, x, y) \mathbb{E}\left[e^{-\lambda_{D} S_{t}}\right] \leq c_{3} h(1, x, y) e^{-t \phi\left(\lambda_{D}\right)} .
\end{aligned}
$$

On the other hand, we also see from Remark 3.7 that

$$
q(t, x, y) \geq c_{4} h(1, x, y) \int_{s_{0}}^{\infty} e^{-\lambda_{D} s} \mathbb{P}\left(S_{t} \in d s\right)=c_{4} h(1, x, y)\left(e^{-t \phi\left(\lambda_{D}\right)}-\int_{0}^{s_{0}} e^{-\lambda_{D} s} \mathbb{P}\left(S_{t} \in d s\right)\right) .
$$


According to Proposition 2.3 and (4.22), it holds that for all $t \geq T$,

$$
\int_{0}^{s_{0}} e^{-\lambda_{D} s} \mathbb{P}\left(S_{t} \in d s\right) \leq \mathbb{P}\left(S_{t} \leq s_{0}\right) \leq \exp \left(-2 t \phi\left(\lambda_{D}\right)\right) .
$$

Therefore, we conclude that for all $t \geq T, q(t, x, y) \geq c_{4}\left(1-e^{-T \phi\left(\lambda_{D}\right)}\right) h(1, x, y) e^{-t \phi\left(\lambda_{D}\right)}$.

\section{Green function estimates}

In this section, we always assume that either (1) (Poly- $\left.R_{1}\right)$ and $\mathbf{H} \mathbf{K}_{\mathbf{B}}^{\mathbf{h}}$ hold, or (2) (Poly- $\infty$ ) and $\mathbf{H K}_{\mathbf{U}}^{\mathbf{h}}$ hold. The Green function $G_{D}$ of $X$ is given by $G_{D}(x, y):=\int_{0}^{\infty} q(t, x, y) d t$.

As an application of the heat kernel estimates obtained in the previous section, we can obtain two-sided estimates on the Green function. To this end, we prove a simple lemma first.

Lemma 5.1. Let $f: I \rightarrow[0, \infty)$ be a function defined on an interval $I \subset[0, \infty)$. Assume that there exist constants $c_{1}, c_{2}>0, p_{1}, p_{2} \in \mathbb{R}$ such that

$$
c_{1}\left(\frac{r_{2}}{r_{1}}\right)^{p_{1}} \leq \frac{f\left(r_{2}\right)}{f\left(r_{1}\right)} \leq c_{2}\left(\frac{r_{2}}{r_{1}}\right)^{p_{2}} \text { for all } r_{1}, r_{2} \in I, 0<r_{1} \leq r_{2} .
$$

For any $a>1$, there exists a constant $c_{3}>0$ such that for all $r, R \in I$, ar $\leq R$,

$$
\int_{r}^{R} s^{-1} f(s) d s \geq c_{3}(f(r)+f(R)) .
$$

(i) If we assume $p_{1}>0$, then, for any $a>1, \int_{r}^{R} s^{-1} f(s) d s \simeq f(R)$ for all $r, R \in I$, ar $\leq R$, with comparison constants depending on a.

(ii) If we assume $p_{2}<0$, then, for any $a>1, \int_{r}^{R} s^{-1} f(s) d s \simeq f(r)$ for all $r, R \in I, 0<$ ar $\leq R$, with comparison constants depending on a.

Proof. Suppose $a>1$. For all $r, R \in I$, ar $\leq R$, by (5.1),

$$
\int_{r}^{R} s^{-1} f(s) d s \geq \int_{r}^{a r} s^{-1} f(s) d s \vee \int_{R / a}^{R} s^{-1} f(s) d s \geq c(f(r) \vee f(R)) .
$$

Thus we only need to prove the upper bounds in (i) and (ii).

(i) By (5.1), since $p_{1}>0$, we have that

$$
\int_{r}^{R} s^{-1} f(s) d s=f(R) \int_{r}^{R} \frac{f(s)}{s f(R)} d s \leq c_{1}^{-1} f(R) \int_{r}^{R} \frac{s^{p_{1}-1}}{R^{p_{1}}} d s \leq c_{1}^{-1} p_{1}^{-1} f(R) .
$$

(ii) Similarly, by (5.1), since $p_{2}<0$, we have that

$$
\int_{r}^{R} s^{-1} f(s) d s=f(r) \int_{r}^{R} \frac{f(s)}{s f(r)} d s \leq c_{2} f(r) \int_{r}^{R} \frac{s^{p_{2}-1}}{r^{p_{2}}} d s \leq-c_{2} p_{2}^{-1} f(r) .
$$

Recall the definition of $\psi$ in (4.8). The following simple observation will be used in the proof of the next proposition and also later.

Lemma 5.2. If $D$ is bounded, then there exists $c>0$ such that for any $x, y \in D$,

$$
\int_{\Phi(\rho(x, y))}^{2 \Phi(\operatorname{diam}(D))} \frac{h(s, x, y)}{s V\left(x, \Phi^{-1}(s)\right) \phi(1 / s)} d s \geq c\left(\frac{h(\Phi(\rho(x, y)), x, y) \psi(\rho(x, y))}{V(x, \rho(x, y))}+h(1, x, y)\right) .
$$

Proof. This follows easily from (5.2), (H1), (H2) and (3.2).

The following proposition provides the first and most general estimate of the Green function.

Proposition 5.3. It holds that for $x, y \in D$,

$$
G_{D}(x, y) \simeq \frac{C_{0}}{V(x, \rho(x, y)) \Psi(\rho(x, y))} \int_{0}^{\Phi(\rho(x, y))} \frac{h(s, x, y)}{\phi(1 / s)} d s+\int_{\Phi(\rho(x, y))}^{2 \Phi(\operatorname{diam}(D))} \frac{h(s, x, y)}{s V\left(x, \Phi^{-1}(s)\right) \phi(1 / s)} d s .
$$


Proof. Since the proofs are similar, we only give the proof when $\mathbf{H K}_{\mathbf{B}}^{\mathbf{h}}$ holds, which is more complicated.

Take $x, y \in D$ and let $r:=\rho(x, y)$. Set $T_{D}:=1 / \phi(1 /(2 \Phi(\operatorname{diam}(D))))$. By a change of variables and Lemma 2.1, we have that

$$
\int_{0}^{\psi(r)} \frac{h\left(\phi^{-1}(1 / t)^{-1}, x, y\right)}{\phi^{-1}(1 / t)} d t=\int_{0}^{\Phi(r)} \frac{h(s, x, y) \phi^{\prime}(1 / s)}{s \phi(1 / s)^{2}} d s \simeq \int_{0}^{\Phi(r)} \frac{h(s, x, y)}{\phi(1 / s)} d s
$$

and

$$
\begin{aligned}
\int_{\psi(r)}^{T_{D}} \frac{h\left(\phi^{-1}(1 / t)^{-1}, x, y\right)}{V\left(x, \psi^{-1}(t)\right)} d t & =\int_{\Phi(r)}^{2 \Phi(\operatorname{diam}(D))} \frac{h(s, x, y)}{V\left(x, \Phi^{-1}(s)\right)} \frac{\phi^{\prime}(1 / s)}{s^{2} \phi(1 / s)^{2}} d s \\
& \simeq \int_{\Phi(r)}^{2 \Phi(\operatorname{diam}(D))} \frac{h(s, x, y)}{s V\left(x, \Phi^{-1}(s)\right) \phi(1 / s)} d s .
\end{aligned}
$$

Combining with Theorem 4.2 (with $T=T_{D}$ ), we arrive at the lower bound in (5.3).

By Theorems 4.2 and 4.7 ( with $T=T_{D}$ ), we have that

$$
\begin{aligned}
G_{D}(x, y) \leq & c_{0} \int_{0}^{\psi(r)} \frac{h\left(\phi^{-1}(1 / t)^{-1}, x, y\right)}{V\left(x, \psi^{-1}(t)\right)} \exp \left(-\frac{c_{1} r^{2}}{\psi^{-1}(t)^{2}}\right) d t+c_{0} h(\Phi(r), x, y) \frac{w(\Phi(r))}{V(x, r)} \int_{0}^{\psi(r)} t d t \\
& +c_{0} \frac{C_{0}}{V(x, r) \Psi(r)} \int_{0}^{\psi(r)} t \mathcal{B}_{h}(t, x, y) d t+c_{0} \frac{C_{0}}{V(x, r) \Psi(r)} \int_{0}^{\psi(r)} \frac{h\left(\phi^{-1}(1 / t)^{-1}, x, y\right)}{\phi^{-1}(1 / t)} d t \\
& +c_{0} \int_{\psi(r)}^{T_{D}} \frac{h\left(\phi^{-1}(1 / t)^{-1}, x, y\right)}{V\left(x, \psi^{-1}(t)\right)} d t+c_{0} h(1, x, y) \int_{T_{D}}^{\infty} e^{-t \phi\left(\lambda_{D}\right)} d t \\
= & : c_{0}\left(G_{1}+G_{2}+C_{0} G_{3}+C_{0} G_{4}+G_{5}+G_{6}\right) .
\end{aligned}
$$

First note that, following the proof of Lemma 3.2 and with help of (4.9), one can see that under the assumptions of Lemma 3.2, there exists $c_{1}>0$ such that for all $r, \kappa \in\left(0, T_{D}\right)$,

$$
\int_{0}^{r} f(s) \exp \left(-\frac{\kappa^{2}}{\psi^{-1}(s)^{2}}\right) d s \leq \frac{c_{1} r^{p+1} f(r)}{\psi(\kappa)^{p}} .
$$

Applying this inequality with $f(t)=h\left(\phi^{-1}(1 / t)^{-1}, x, y\right) V\left(x, \psi^{-1}(t)\right)^{-1}$ and $p:=\gamma / \beta_{1}+d_{2} /\left(\alpha_{1} \beta_{1}\right)$, we get from (H2), (2.4), (3.1) and (4.9) that

$$
G_{1} \leq c_{2} \frac{h(\Phi(r), x, y)}{V(x, r)} \frac{\psi(r)^{p+1}}{\psi(r)^{p}}=c_{2} h(\Phi(r), x, y) \frac{\psi(r)}{V(x, r)} .
$$

For $G_{2}$, we see from (2.10) that

$$
G_{2} \leq \frac{e}{2(e-2)} h(\Phi(r), x, y) \frac{\psi(r)^{2}}{V(x, r) \psi(r)}=\frac{e}{2(e-2)} h(\Phi(r), x, y) \frac{\psi(r)}{V(x, r)} .
$$

For $G_{3}$, we use Fubini's theorem to get that

$$
\begin{aligned}
V(x, r) \Psi(r) G_{3} & =\int_{0}^{\psi(r)} t \int_{2 \phi^{-1}(1 / t)^{-1}}^{4 \Phi(r)} h(s, x, y) w(s) d s d t \\
& =\int_{0}^{2 \Phi(r)} h(s, x, y) w(s) \int_{0}^{\phi(2 / s)^{-1}} t d t d s+\int_{2 \Phi(r)}^{4 \Phi(r)} h(s, x, y) w(s) \int_{0}^{\psi(r)} t d t d s \\
& =: G_{3,1}+G_{3,2} .
\end{aligned}
$$

By (2.10), a change of variables and (H2), we get

$$
G_{3,1} \leq c_{3} \int_{0}^{2 \Phi(r)} \frac{h(s, x, y) \phi(2 / s)}{\phi(2 / s)^{2}} d s=2 c_{3} \int_{0}^{\Phi(r)} \frac{h(2 s, x, y)}{\phi(1 / s)} d s \leq c_{4} \int_{0}^{\Phi(r)} \frac{h(s, x, y)}{\phi(1 / s)} d s .
$$

On the other hand, we also get from (2.10), (H1) and (2.2) that

$$
\begin{aligned}
G_{3,2} & \leq \frac{e}{2(e-2)} h(\Phi(r), x, y) \phi(1 / \Phi(r)) \psi(r)^{2} \int_{2 \Phi(r)}^{4 \Phi(r)} d s=\frac{e}{(e-2)} h(\Phi(r), x, y) \psi(r) \Phi(r) \\
& \leq \frac{2 e}{(e-2)} \frac{\psi(r)}{\phi(2 / \Phi(r))^{-1}} \int_{\Phi(r) / 2}^{\Phi(r)} \frac{h(s, x, y)}{\phi(1 / s)} d s \leq c_{5} \int_{\Phi(r) / 2}^{\Phi(r)} \frac{h(s, x, y)}{\phi(1 / s)} d s \leq c_{5} \int_{0}^{\Phi(r)} \frac{h(s, x, y)}{\phi(1 / s)} d s .
\end{aligned}
$$


Clearly, $G_{6} \leq \phi\left(\lambda_{D}\right)^{-1} h(1, x, y)$.

Recall from (5.4) and (5.5) that

$$
G_{4}+G_{5} \simeq \frac{C_{0}}{V(x, r) \Psi(r)} \int_{0}^{\Phi(r)} \frac{h(s, x, y)}{\phi(1 / s)} d s+\int_{\Phi(r)}^{2 \Phi(\operatorname{diam}(D))} \frac{h(s, x, y)}{s V\left(x, \Phi^{-1}(s)\right) \phi(1 / s)} d s .
$$

It follows from Lemma 5.2 and the upper bounds above on $G_{1}, G_{2}, G_{6}$ that $G_{5}$ dominates $G_{1}+G_{2}+G_{6}$. Since $G_{4}$ dominates $G_{3}$, the proof is complete.

In the remainder of this section, under some additional assumptions on the boundary function, we will obtain Green function estimates in simpler forms. Lemma 5.1] will be a useful tool in all simplifications.

We start with the following condition which is a counterpart of (H2).

$(\mathrm{H} 2 *)$ There exist constants $c_{1}, \gamma_{*}>0$ such that for all $x, y \in D$ and $s, t \geq 0$ with $\Phi\left(\delta_{\vee}(x, y)\right) \leq s \leq t<$ $2 \Phi(\operatorname{diam}(D))$,

$$
s^{\gamma_{*}} h(s, x, y) \geq c_{1} t^{\gamma_{*}} h(t, x, y) .
$$

Note that the $\gamma_{*}$ above is less than or equal to $\gamma$.

Remark 5.4. Suppose that the boundary function $h(t, x, y)$ satisfies $\left(H 2^{*}\right)$. Then for every $\epsilon \in(0,1)$, there exists $c_{2}=c_{2}(\epsilon)>0$ such that for all $x, y \in D$ and $s, t \geq 0$ with $\epsilon \Phi\left(\delta_{\vee}(x, y)\right) \leq s \leq t<$ $2 \Phi(\operatorname{diam}(D))$,

$$
s^{\gamma_{*}} h(s, x, y) \geq c_{2} t^{\gamma_{*}} h(t, x, y) .
$$

Indeed, let $\epsilon \Phi\left(\delta_{\vee}(x, y)\right) \leq s \leq \Phi\left(\delta_{\vee}(x, y)\right)$ and $s \leq t<2 \Phi(\operatorname{diam}(D))$. If $t \leq \Phi\left(\delta_{\vee}(x, y)\right)$, then $\epsilon t \leq s$ so that by $(\mathrm{H} 1)$,

$$
s^{\gamma_{*}} h(s, x, y) \geq s^{\gamma_{*}} h(t, x, y) \geq \epsilon^{\gamma_{*}} t^{\gamma_{*}} h(t, x, y) .
$$

If $t>\Phi\left(\delta_{\vee}(x, y)\right)$, then by using (H1) in the first inequality below, $(\mathrm{H} 2 *)$ in the second, and the condition that $s \geq \epsilon \Phi\left(\delta_{\vee}(x, y)\right)$ in the last inequality, we see that

$$
s^{\gamma_{*}} h(s, x, y) \geq s^{\gamma_{*}} h\left(\Phi\left(\delta_{\vee}(x, y)\right), x, y\right) \geq c_{1}\left(\frac{s}{\Phi\left(\delta_{\vee}(x, y)\right)}\right)^{\gamma_{*}} t^{\gamma_{*}} h(t, x, y) \geq c_{1} \epsilon^{\gamma_{*}} t^{\gamma_{*}} h(t, x, y) .
$$

Example 5.5. Let $p, q \geq 0, p+q>0$. Recall that the boundary function $h_{p, q}(t, x, y)$ defined in (3.8) satisfies (H2) with $\gamma=p+q$. We claim that $h_{p, q}(t, x, y)$ also satisfies $\left(\mathrm{H} 2^{*}\right)$ with $\gamma_{*}=\gamma=p+q$. Indeed, for all $x, y \in D$ and $\Phi\left(\delta_{\vee}(x, y)\right)<s<t$,

$$
s^{p+q} h_{p, q}(s, x, y)=\Phi\left(\delta_{D}(x)\right)^{p} \Phi\left(\delta_{D}(y)\right)^{q}=t^{p+q} h_{p, q}(t, x, y) .
$$

In the remainder of this section, we let $d_{1}, d_{2}, \gamma, \gamma_{*}, \beta_{1}, \beta_{2}$ and $\alpha_{1}, \alpha_{2}$ be the constants in (3.1), (H2), $\left(\mathrm{H} 2^{*}\right),\left(\right.$ Poly- $\left.R_{1}\right)$ and the scaling indices of $\Phi$ in (3.4), respectively.

Let

$$
\widetilde{G}_{D}(x, y):=\int_{\Phi(\rho(x, y))}^{2 \Phi(\operatorname{diam}(D))} \frac{h(s, x, y)}{s V\left(x, \Phi^{-1}(s)\right) \phi(1 / s)} d s
$$

denote the second term on the right-hand side of the estimate (5.3).

Lemma 5.6. The following estimates hold for all $x, y \in D$.

(i) If $d_{1}>\alpha_{2}\left(\beta_{2} \wedge 1\right)$, then

$$
\widetilde{G}_{D}(x, y) \simeq h(\Phi(\rho(x, y)), x, y) \frac{\psi(\rho(x, y))}{V(x, \rho(x, y))} .
$$

(ii) If $d_{2}<\alpha_{1}\left(\beta_{1}-\gamma\right)$, then

$$
\widetilde{G}_{D}(x, y) \simeq \begin{cases}h(1, x, y), & \text { when } \mathbf{H K}_{\mathbf{B}}^{\mathbf{h}} \text { holds } \\ \infty, & \text { when } \mathbf{H K}_{\mathbf{U}}^{\mathbf{h}} \text { holds }\end{cases}
$$

Below, we also assume that $h(t, x, y)$ is regular and $\left(\mathrm{H}^{*}{ }^{*}\right)$ holds.

(iii) If $\alpha_{1} \beta_{1}>d_{2} \geq d_{1}>\alpha_{2}\left(\left(\beta_{2} \wedge 1\right)-\gamma_{*}\right)$, then

$$
\widetilde{G}_{D}(x, y) \simeq h(\Phi(\rho(x, y)), x, y) \frac{\psi\left(\rho(x, y) \vee \delta_{\vee}(x, y)\right)}{V\left(x, \rho(x, y) \vee \delta_{\vee}(x, y)\right)}
$$


(iv) If $d_{1}=d_{2}=\alpha_{1} \beta_{1}=\alpha_{2} \beta_{2}$, then

$$
\widetilde{G}_{D}(x, y) \simeq h(\Phi(\rho(x, y)), x, y) \log \left(e+\frac{\delta_{\vee}(x, y)}{\rho(x, y)}\right) .
$$

(v) If $\alpha_{1}=\alpha_{2}, \beta_{1}=\beta_{2}, \gamma=\gamma_{*}$ and $d_{1}=d_{2}=\alpha_{1}\left(\beta_{1}-\gamma\right)$, then

$$
\widetilde{G}_{D}(x, y) \simeq \begin{cases}h(1, x, y) \log \left(e+\operatorname{diam}(D)\left(\rho(x, y) \vee \delta_{\vee}(x, y)\right)^{-1}\right), & \text { when } \mathbf{H K}_{\mathbf{B}}^{\mathbf{h}} \text { holds }, \\ \infty, & \text { when } \mathbf{H K}_{\mathbf{U}}^{\mathbf{h}} \text { holds }\end{cases}
$$

Proof. Take $x, y \in D$. Let $\delta_{\wedge}:=\delta_{\wedge}(x, y)$ and $\delta_{\vee}:=\delta_{\vee}(x, y)$. Define

$$
g(s):=\frac{h(s, x, y)}{V\left(x, \Phi^{-1}(s)\right) \phi(1 / s)}, \quad s>0 .
$$

Then

$$
\widetilde{G}_{D}(x, y)=\int_{\Phi(\rho(x, y))}^{2 \Phi(\operatorname{diam}(D))} \frac{g(s)}{s} d s .
$$

By (H1), (H2), (3.1), (3.4), (2.2) and (2.3), there exist $c_{1}, c_{2}>0$ such that

$$
c_{1}\left(\frac{r}{s}\right)^{-\gamma-d_{2} / \alpha_{1}+\beta_{1}} \leq \frac{g(r)}{g(s)} \leq c_{2}\left(\frac{r}{s}\right)^{-d_{1} / \alpha_{2}+\left(\beta_{2} \wedge 1\right)}, \quad 0<s \leq r<2 \Phi(\operatorname{diam}(D)) .
$$

If $h(t, x, y)$ is regular, then by Remark [3.4, for every $a>0$, there exists $c_{3}=c_{3}(a)>0$ such that

$$
c_{3}\left(\frac{r}{s}\right)^{-d_{2} / \alpha_{1}+\beta_{1}} \leq \frac{g(r)}{g(s)} \leq c_{2}\left(\frac{r}{s}\right)^{-d_{1} / \alpha_{2}+\left(\beta_{2} \wedge 1\right)}, 0<s \leq r<\Phi\left(a \delta_{\wedge}\right) \wedge 2 \Phi(\operatorname{diam}(D)) ;
$$

if furthermore $\left(\mathrm{H}_{2} *\right)$ further holds, then by Remark 5.4, there exists $c_{4}>0$ such that

$$
c_{1}\left(\frac{r}{s}\right)^{-\gamma-d_{2} / \alpha_{1}+\beta_{1}} \leq \frac{g(r)}{g(s)} \leq c_{4}\left(\frac{r}{s}\right)^{-\gamma_{*}-d_{1} / \alpha_{2}+\left(\beta_{2} \wedge 1\right)}, \quad \Phi\left(\delta_{\vee} / 2\right)<s \leq r<2 \Phi(\operatorname{diam}(D)) .
$$

(i) By (5.8), since $-d_{1} / \alpha_{2}+\left(\beta_{2} \wedge 1\right)<0$, the result follows from Lemma 5.1(ii).

(ii) If $D$ is bounded, then by (5.8) and Lemma 5.1(i), since $-\gamma-d_{2} / \alpha_{1}+\beta_{1}>0$, it holds that $\widetilde{G}_{D}(x, y) \simeq g(\Phi(\operatorname{diam}(D)))$. By (3.2), there exists a constant $c_{5}>1$ such that $c_{5}^{-1} \leq V(z, \operatorname{diam}(D)) \leq c_{5}$ for all $z \in D$. Hence, by using (H1), (H2) and the definition of $g$, we get that $G_{D}(x, y) \simeq h(1, x, y)$. If $D$ is unbounded, then we see from (5.8) and Lemma 5.1(i) that

$$
\widetilde{G}_{D}(x, y) \simeq \lim _{r \rightarrow \infty} \int_{\Phi(\rho(x, y))}^{r} \frac{g(s)}{s} d s \simeq \lim _{r \rightarrow \infty} g(r) \geq c_{1} g(1) \lim _{r \rightarrow \infty} r^{-\gamma-d_{2} / \alpha_{1}+\beta_{1}}=\infty .
$$

(iii) Suppose that $\delta_{\vee} \leq 2 \rho(x, y)$. Since $-\gamma_{*}-d_{1} / \alpha_{2}+\left(\beta_{2} \wedge 1\right)<0$, by (5.10) and Lemma 5.1(ii),

$$
\widetilde{G}_{D}(x, y) \simeq g(\Phi(\rho(x, y)))=\frac{h(\Phi(\rho(x, y)), x, y) \psi(\rho(x, y))}{V(x, \rho(x, y))} .
$$

Hence the result follows from (3.1) and (4.9).

Suppose now that $\delta_{\vee}>2 \rho(x, y)$. Then $\delta_{\wedge} \geq \delta_{\vee}-\rho(x, y)>\delta_{\vee} / 2>\rho(x, y)$. Since $h$ is regular, we get $h\left(\Phi\left(\delta_{\vee}\right), x, y\right) \simeq h(\Phi(\rho(x, y)), x, y) \simeq 1$. Further, since $-d_{2} / \alpha_{1}+\beta_{1}>0$ and $-\gamma_{*}-d_{1} / \alpha_{2}+\left(\beta_{2} \wedge 1\right)<0$, by the scaling of $\Phi,(5.9)$, (5.10) and Lemma 5.1(i)-(ii), we get

$$
\begin{aligned}
\widetilde{G}_{D}(x, y) & \simeq \int_{\Phi(\rho(x, y))}^{2 \Phi\left(\delta_{\wedge}\right)} \frac{g(s)}{s} d s+\int_{\Phi\left(\delta_{\vee}\right)}^{2 \Phi(\operatorname{diam}(D))} \frac{g(s)}{s} d s \simeq g\left(\Phi\left(\delta_{\wedge}\right)\right)+g\left(\Phi\left(\delta_{\vee}\right)\right) \\
& \simeq g\left(\Phi\left(\delta_{\vee}\right)\right)=\frac{h\left(\Phi\left(\delta_{\vee}\right), x, y\right) \psi\left(\delta_{\vee}\right)}{V\left(x, \delta_{\vee}\right)} \simeq \frac{\psi\left(\delta_{\vee}\right)}{V\left(x, \delta_{\vee}\right)} \simeq \frac{h(\Phi(\rho(x, y)), x, y) \psi\left(\delta_{\vee}\right)}{V\left(x, \delta_{\vee}\right)} .
\end{aligned}
$$

This finishes the proof for (iii).

(iv) Since $d_{1}=d_{2}$, by (3.1) and (3.2), we see that for every $a>0$, there are comparability constants depending on $a$ such that for all $w, z \in D$ and $0<r<a \operatorname{diam}(D)$,

$$
V(w, r) \simeq\left(\frac{r}{\rho(w, z)}\right)^{d_{1}} V(w, \rho(w, z)) \simeq\left(\frac{r}{\rho(w, z)}\right)^{d_{1}} V(z, \rho(w, z)) \simeq V(z, r) \simeq r^{d_{1}} V(z, 1) .
$$

Moreover, since $\beta_{1}=\beta_{2}$ and $\alpha_{1}=\alpha_{2}$, by (2.2), (2.3) and (3.4), we get that

$$
\phi(1 / s)^{-1} \simeq s^{\beta_{1}}, \quad 0<s<2 \Phi(\operatorname{diam}(D)) \quad \text { and } \quad \Phi^{-1}(s) \simeq s^{1 / \alpha_{1}}, \quad s>0,
$$


so that $g(s) \simeq h(s, x, y)$ for all $0<s<2 \Phi(\operatorname{diam}(D))$. In particular, since $h$ is regular, we see from Remark 3.4 that

$$
g(s) \simeq 1, \quad 0<s<2 \Phi\left(\delta_{\wedge}\right) .
$$

If $\delta_{\vee} \leq 2 \rho(x, y)$, then by (5.10) and Lemma 5.1(ii),

$$
\widetilde{G}_{D}(x, y) \simeq g(\Phi(\rho(x, y))) \simeq h(\Phi(\rho(x, y)), x, y) \simeq h(\Phi(\rho(x, y)), x, y) \log \left(e+\frac{\delta_{\vee}}{\rho(x, y)}\right) .
$$

If $\delta_{\vee}>2 \rho(x, y)$, then we get $\delta_{\wedge}>\delta_{\vee} / 2>\rho(x, y)$ as in (iii), and by (5.13), (5.10) and Lemma 5.1(ii),

$$
\widetilde{G}_{D}(x, y) \simeq \int_{\Phi(\rho(x, y))}^{2 \Phi\left(\delta_{\wedge}\right)} \frac{g(s)}{s} d s+\int_{\Phi\left(\delta_{\vee}\right)}^{2 \Phi(\operatorname{diam}(D))} \frac{g(s)}{s} d s \simeq \int_{\Phi(\rho(x, y))}^{2 \Phi\left(\delta_{\wedge}\right)} \frac{d s}{s}+g\left(\Phi\left(\delta_{\vee}\right)\right) .
$$

Note that since $\Phi(s) \simeq s^{\alpha_{1}}$ for $s>0$, we have $\int_{\Phi(\rho(x, y))}^{2 \Phi\left(\delta_{\wedge}\right)} s^{-1} d s \simeq \log \left(e+\frac{\delta_{\wedge}}{\rho(x, y)}\right)$ so that

$$
g\left(\Phi\left(\delta_{\vee}\right)\right) \simeq h\left(\Phi\left(\delta_{\vee}\right), x, y\right) \leq 1 \leq \log \left(e+\frac{\delta_{\wedge}}{\rho(x, y)}\right) \simeq \int_{\Phi(\rho(x, y))}^{2 \Phi\left(\delta_{\wedge}\right)} \frac{d s}{s} .
$$

Eventually, since $\delta_{\wedge} \simeq \delta_{\vee}$ and $h(\Phi(\rho(x, y)), x, y) \simeq 1$ in this case, we obtain that

$$
\widetilde{G}_{D}(x, y) \simeq \log \left(e+\frac{\delta_{\wedge}}{\rho(x, y)}\right) \simeq h(\Phi(\rho(x, y)), x, y) \log \left(e+\frac{\delta_{\vee}}{\rho(x, y)}\right) .
$$

(v) By (5.11), (5.12), the regularity of $h,(\mathrm{H} 2)$, Remark 5.4 and (3.10), we have

$$
g(s) \simeq s^{\gamma}, \quad 0<s<\Phi\left(\delta_{\wedge}\right)
$$

and

$$
g(s) \simeq s^{\gamma} h(s, x, y) \simeq t^{\gamma} h(t, x, y), \quad \Phi\left(\delta_{\vee} / 2\right)<s \leq t<2 \Phi(\operatorname{diam}(D))+1 .
$$

If $\delta_{\vee} \leq 2 \rho(x, y)$, then since $\Phi(s) \simeq s^{\alpha_{1}}$ for $s>0$ in this case, we get from (5.15) that

$$
\widetilde{G}_{D}(x, y) \simeq h(1, x, y) \int_{\Phi(\rho(x, y))}^{2 \Phi(\operatorname{diam}(D))} \frac{d s}{s} \simeq h(1, x, y) \log \left(e+\frac{\operatorname{diam}(D)}{\rho(x, y)}\right) .
$$

If $\delta_{\vee}>2 \rho(x, y)$, then $\delta_{\wedge}>\delta_{\vee} / 2>\rho(x, y)$ as in (iii) and hence by (5.14) and (5.15),

$$
\widetilde{G}_{D}(x, y) \simeq \int_{\Phi(\rho(x, y))}^{2 \Phi\left(\delta_{\wedge}\right)} s^{\gamma-1} d s+h(1, x, y) \int_{\Phi\left(\delta_{\vee}\right)}^{2 \Phi(\operatorname{diam}(D))} \frac{d s}{s} .
$$

Since $\Phi(s) \simeq s^{\alpha_{1}}$ for $s>0, \delta_{\wedge} \leq \delta_{\vee} \leq 2 \delta_{\wedge}$ and $h$ is regular, by (5.15),

$$
\begin{aligned}
& h(1, x, y) \int_{\Phi\left(\delta_{\vee}\right)}^{2 \Phi(\operatorname{diam}(D))} \frac{d s}{s} \simeq h(1, x, y) \log \left(e+\frac{\operatorname{diam}(D)}{\delta_{\vee}}\right) \\
& \geq h(1, x, y) \simeq \Phi\left(\delta_{\wedge}\right)^{\gamma} h\left(\Phi\left(\delta_{\wedge}\right), x, y\right) \simeq \Phi\left(\delta_{\wedge}\right)^{\gamma} \geq \gamma^{-1} \int_{\Phi(\rho(x, y))}^{2 \Phi\left(\delta_{\wedge}\right)} s^{\gamma-1} d s .
\end{aligned}
$$

This completes the proof.

In the next lemma we show that under the additional assumption that $\gamma<\beta_{1}+1$, the first term on the right-hand side of (5.3) is dominated by $\widetilde{G}_{D}(x, y)$.

Lemma 5.7. If either $C_{0}=0$ or $\gamma<\beta_{1}+1$, then $G_{D}(x, y) \simeq \widetilde{G}_{D}(x, y)$ on $D \times D$.

Proof. When $C_{0}=0$, the assertion follows from Proposition 5.3. So we now assume $\gamma<\beta_{1}+1$. According to (2.3) and (H2), we have

$$
\frac{h(t, x, y) \phi(1 / t)^{-1}}{h(s, x, y) \phi(1 / s)^{-1}} \geq c\left(\frac{t}{s}\right)^{\beta_{1}-\gamma} \text { for all } 0<s \leq t<\Phi(\operatorname{diam}(D)) .
$$

Thus, since $\Psi \geq \Phi$, by Lemma 5.1(i), (H1) and (2.2), we get

$$
\frac{C_{0}}{V(x, r) \Psi(r)} \int_{0}^{\Phi(r)} \frac{h(s, x, y)}{\phi(1 / s)} d s \leq c \frac{h(\Phi(r), x, y) \psi(r) \Phi(r)}{V(x, r) \Psi(r)} \leq c \frac{h(\Phi(r), x, y) \psi(r)}{V(x, r)} .
$$

Combining the above with Lemma 5.2 and Proposition 5.3, we get the assertion. 
Define

$$
g_{0}(x, y)= \begin{cases}\frac{\psi(\rho(x, y))}{V(x, \rho(x, y))}, & \text { if } d_{1}>\alpha_{2}\left(\beta_{2} \wedge 1\right), \\ \log \left(e+\frac{\delta_{\vee}(x, y)}{\rho(x, y)}\right), & \text { if } d_{1}=d_{2}=\alpha_{1} \beta_{1}=\alpha_{2} \beta_{2}, \\ \frac{\psi\left(\rho(x, y) \vee \delta_{\vee}(x, y)\right)}{V\left(x, \rho(x, y) \vee \delta_{\vee}(x, y)\right)}, & \text { if } d_{2}<\alpha_{1} \beta_{1} .\end{cases}
$$

By combining Proposition 5.3, Lemma 5.6 and Lemma 5.7 we arrive at the following result.

Theorem 5.8. Suppose that $C_{0}=0$ or $\gamma<\beta_{1}+1, h(t, x, y)$ is regular and (H2*) holds.

(a) Suppose also that one of the following holds: (1) $d_{1}>\alpha_{2}\left(\beta_{2} \wedge 1\right)$ or (2) $d_{1}=d_{2}=\alpha_{1} \beta_{1}=\alpha_{2} \beta_{2}$ or (3) $d_{2}<\alpha_{1} \beta_{1}$. Then it holds that

$$
G_{D}(x, y) \simeq h(\Phi(\rho(x, y)), x, y) g_{0}(x, y) .
$$

(b) If $d_{2}<\alpha_{1}\left(\beta_{1}-\gamma\right)$, then

$$
G_{D}(x, y) \simeq \begin{cases}h(1, x, y), & \text { when } \mathbf{H K}_{\mathbf{B}}^{\mathbf{h}} \text { holds } \\ \infty, & \text { when } \mathbf{H K}_{\mathbf{U}}^{\mathbf{h}} \text { holds }\end{cases}
$$

(c) If $\alpha_{1}=\alpha_{2}, \beta_{1}=\beta_{2}, \gamma=\gamma_{*}$ and $d_{1}=d_{2}=\alpha_{1}\left(\beta_{1}-\gamma\right)$, then

$$
G_{D}(x, y) \simeq \begin{cases}h(1, x, y) \log \left(e+\operatorname{diam}(D)\left(\rho(x, y) \vee \delta_{\vee}(x, y)\right)^{-1}\right), & \text { when } \mathbf{H K}_{\mathbf{B}}^{\mathbf{h}} \text { holds }, \\ \infty, & \text { when } \mathbf{H K}_{\mathbf{U}}^{\mathbf{h}} \text { holds. }\end{cases}
$$

When $C_{0}=1$, Theorem 5.8 only deals with the case $\gamma<\beta_{1}+1$. To cover the case when $\gamma$ is large, we assume the following condition.

$\left(\mathrm{H} 2^{* *}\right)$ There exist constants $c_{1}>0, \gamma_{* *} \in\left(0,1+\beta_{1}\right)$ such that for all $x, y \in D$ and $s, t \geq 0$ with $\Phi\left(\delta_{\wedge}(x, y)\right) \leq s \leq t<\Phi\left(\delta_{\vee}(x, y)\right)$,

$$
s^{\gamma_{* *}} h(s, x, y) \leq c_{1} t^{\gamma_{* *}} h(t, x, y) .
$$

Example 5.9. For $p, q \geq 0$, let $h_{p, q}(t, x, y)$ be the boundary function defined in (3.8). If $p \vee q<1+\beta_{1}$, then $h_{p, q}(t, x, y)$ satisfies $\left(\mathrm{H} 2^{* *}\right)$. Indeed, we see that for all $x, y \in D$ and $\Phi\left(\delta_{\wedge}(x, y)\right) \leq s \leq t<\Phi\left(\delta_{\vee}(x, y)\right)$,

$$
s^{p \vee q} h_{p, q}(s, x, y)=\left\{\begin{array}{ll}
\Phi\left(\delta_{D}(x)\right)^{p} s^{p \vee q-p}, & \text { if } \delta_{D}(x)<\delta_{D}(y) \\
\Phi\left(\delta_{D}(y)\right)^{q} s^{p \vee q-q}, & \text { if } \delta_{D}(x)>\delta_{D}(y)
\end{array} \leq t^{p \vee q} h_{p, q}(t, x, y) .\right.
$$

For a given boundary function $h$, we define for $x, y \in D$,

$$
[h](x, y):=h\left(\Phi\left(\rho(x, y) \wedge \delta_{\vee}(x, y)\right), x, y\right)\left(1 \wedge \frac{\Phi\left(\delta_{\vee}(x, y)\right) \psi\left(\delta_{\vee}(x, y)\right)}{\Phi(\rho(x, y)) \psi(\rho(x, y))}\right) .
$$

One can see that for all $p, q \geq 0$,

$$
\left[h_{p, q}\right](x, y) \simeq\left(1 \wedge \frac{\Phi\left(\delta_{D}(x)\right)}{\Phi(\rho(x, y))}\right)^{p}\left(1 \wedge \frac{\Phi\left(\delta_{D}(y)\right)}{\Phi(\rho(x, y))}\right)^{q}\left(1 \wedge \frac{\Phi\left(\delta_{\vee}(x, y)\right)}{\Phi(\rho(x, y))}\right)^{1-p-q}\left(1 \wedge \frac{\psi\left(\delta_{\vee}(x, y)\right)}{\psi(\rho(x, y))}\right) .
$$

Indeed, for $x, y \in D$, if $\delta_{\wedge}(x, y) \geq \rho(x, y)$, then $\left[h_{p, q}\right](x, y)=1$ and the right-hand side of (5.18) is also equal to 1. If $\delta_{\wedge}(x, y)<\rho(x, y)$, then $\delta_{\vee}(x, y)<\rho(x, y)+\delta_{\wedge}(x, y) \leq \rho(x, y)+\rho(x, y) \leq 2 \rho(x, y)$ and hence by (3.10) and the scaling properties of $\Phi$ and $\psi$,

$$
\begin{aligned}
\frac{\Phi(\rho(x, y))^{1-p-q} \psi(\rho(x, y))}{\Phi\left(\delta_{\vee}(x, y)\right)^{1-p-q} \psi\left(\delta_{\vee}(x, y)\right)}\left[h_{p, q}\right](x, y) & \simeq \frac{\Phi\left(\delta_{\vee}(x, y)\right)^{p+q}}{\Phi(\rho(x, y))^{p+q}} h_{p, q}\left(\Phi\left(\delta_{\vee}(x, y), x, y\right)=\frac{\Phi\left(\delta_{D}(x)\right)^{p} \Phi\left(\delta_{D}(y)\right)^{q}}{\Phi(\rho(x, y))^{p+q}}\right. \\
& \simeq\left(1 \wedge \frac{\Phi\left(\delta_{D}(x)\right)}{\Phi(\rho(x, y))}\right)^{p}\left(1 \wedge \frac{\Phi\left(\delta_{D}(y)\right)}{\Phi(\rho(x, y))}\right)^{q} .
\end{aligned}
$$

Recall that $g_{0}(x, y)$ is defined in (5.16). 
Theorem 5.10. Suppose that $C_{0}=1, h(t, x, y)$ is regular, $\left(H 2^{*}\right)$ holds with $\gamma_{*}>\left(\beta_{2} \wedge 1\right)+1$ and (H2**) holds. Suppose also that one of the following holds: (1) $d_{1}>\alpha_{2}\left(\beta_{2} \wedge 1\right)$ or (2) $d_{1}=d_{2}=\alpha_{1} \beta_{1}=\alpha_{2} \beta_{2}$ or (3) $d_{2}<\alpha_{1} \beta_{1}$. Then it holds that

$$
G_{D}(x, y) \simeq[h](x, y) \frac{\Phi(\rho(x, y))}{\Psi(\rho(x, y))} \frac{\psi(\rho(x, y))}{V(x, \rho(x, y))}+h(\Phi(\rho(x, y)), x, y) g_{0}(x, y) .
$$

In particular, if $\Psi \simeq \Phi$, then

$$
G_{D}(x, y) \simeq[h](x, y) g_{0}(x, y)
$$

Proof. Take $x, y \in D$ and let $r:=\rho(x, y)$ and $\delta_{\vee}:=\delta_{\vee}(x, y)$. Observe that by (2.2), (2.3), (H1), (H2**) and the regularity of $h$,

$$
c_{1}\left(\frac{t}{s}\right)^{\beta_{1}-\gamma_{* *}} \leq \frac{h(t, x, y) / \phi(1 / t)}{h(s, x, y) / \phi(1 / s)} \leq c_{2}\left(\frac{t}{s}\right)^{\beta_{2} \wedge 1}, \quad 0<s \leq t<\Phi\left(\delta_{\vee}\right) .
$$

Note also that by (2.2), (2.3), (H2), (H2*) and Remark 5.4,

$$
c_{3}\left(\frac{t}{s}\right)^{\beta_{1}-\gamma} \leq \frac{h(t, x, y) / \phi(1 / t)}{h(s, x, y) / \phi(1 / s)} \leq c_{4}\left(\frac{t}{s}\right)^{\beta_{2} \wedge 1-\gamma_{*}}, \quad \Phi\left(\delta_{\vee}\right) / 2 \leq s \leq t<\Phi(\operatorname{diam}(D)) .
$$

If $\delta_{\vee}>2 r$, then by Lemma [5.1(i) and (5.21), $\int_{0}^{\Phi(r)} h(s, x, y) \phi(1 / s)^{-1} d s \simeq h(\Phi(r), x, y) \Phi(r) \psi(r)$.

If $\delta_{\vee} \leq 2 r$, then by Lemma 5.1(i)-(ii), (5.21), (5.22) and the scaling property of $\phi$, since $\beta_{1}-\gamma_{* *}>-1$ and $\beta_{2} \wedge 1-\gamma_{*}<-1$, we get that

$$
\int_{0}^{\Phi(r)} \frac{h(s, x, y)}{\phi(1 / s)} d s=\int_{0}^{\Phi\left(\delta_{\vee}\right) / 2} \frac{h(s, x, y)}{\phi(1 / s)} d s+\int_{\Phi\left(\delta_{\vee}\right) / 2}^{\Phi(r)} \frac{h(s, x, y)}{\phi(1 / s)} d s \simeq h\left(\Phi\left(\delta_{\vee}\right), x, y\right) \Phi\left(\delta_{\vee}\right) \psi\left(\delta_{\vee}\right) .
$$

Therefore, in either case, it holds that

$$
\int_{0}^{\Phi(r)} \frac{h(s, x, y)}{\phi(1 / s)} d s \simeq h\left(\Phi\left(r \wedge \delta_{\vee}\right), x, y\right)\left(1 \wedge \frac{\Phi\left(\delta_{\vee}\right) \psi\left(\delta_{\vee}\right)}{\Phi(r) \psi(r)}\right) \Phi(r) \psi(r)=[h](x, y) \Phi(r) \psi(r) .
$$

Combining this with Proposition 5.3 and Lemma 5.6, we get (5.19).

Now we also assume that $\Psi \simeq \Phi$. If $\delta_{\vee}>r$, then $[h](x, y)=h(\Phi(r), x, y)$. Hence, we see from Lemma 5.2 that in (5.19), the second term dominates the first one so that (5.20) holds. If $\delta_{\mathrm{V}} \leq r$, then using Lemma 5.1(ii), (5.22) and the condition that $\beta_{2} \wedge 1-\gamma_{*}<-1$ in the second inequality below, the scaling property of $\phi$ and (3.10) in the third, and (5.23) in the fourth, we get

$$
\begin{aligned}
& \int_{\Phi(r)}^{2 \Phi(\operatorname{diam}(D))} \frac{h(s, x, y)}{s V\left(x, \Phi^{-1}(s)\right) \phi(1 / s)} d s \leq \frac{1}{V(x, r) \Phi(r)} \int_{\Phi(r)}^{2 \Phi(\operatorname{diam}(D))} \frac{h(s, x, y)}{\phi(1 / s)} d s \\
& \leq c_{5} \frac{h(\Phi(r), x, y) \psi(r)}{V(x, r)} \leq \frac{c_{6}}{V(x, r) \Phi(r)} \int_{\Phi(r) / 2}^{\Phi(r)} \frac{h(s, x, y)}{\phi(1 / s)} d s \leq c_{7}[h](x, y) \frac{\psi(r)}{V(x, r)}
\end{aligned}
$$

Note that $g_{0}(x, y) \simeq \psi(r) / V(x, r)$ when $\delta_{\vee} \leq r$. Thus by Proposition 5.3 and (5.23), we get $G_{D}(x, y) \simeq$ $[h](x, y) \psi(r) / V(x, r) \simeq[h](x, y) g_{0}(x, y)$ when $\delta_{\vee} \leq r$. This completes the proof for (5.20).

For completeness, we record the Green function estimates when $C_{0}=1, \beta_{1}=\beta_{2}$ and $\gamma_{*}=\gamma=\beta_{1}+1$.

Theorem 5.11. Suppose that $C_{0}=1, \beta_{1}=\beta_{2}, h(t, x, y)$ is regular and $\left(H 2^{*}\right)$ holds with $\gamma_{*}=\gamma=\beta_{1}+1$ and $\left(H 2^{* *}\right)$ holds. Suppose also that one of the following holds: (1) $d_{1}>\alpha_{2}\left(\beta_{2} \wedge 1\right)$ or (2) $d_{1}=d_{2}=$ $\alpha_{1} \beta_{1}=\alpha_{2} \beta_{2}$ or (3) $d_{2}<\alpha_{1} \beta_{1}$. Then it holds that

$$
G_{D}(x, y) \simeq h(\Phi(\rho(x, y)), x, y)\left[\frac{\Phi(\rho(x, y))}{\Psi(\rho(x, y)} \frac{\Phi(\rho(x, y))^{\gamma-1}}{V(x, \rho(x, y))} \log \left(e+\frac{\Phi(\rho(x, y))}{\Phi\left(\delta_{\vee}(x, y)\right)}\right)+g_{0}(x, y)\right] .
$$

In particular, if $\Psi \simeq \Phi$, then

$$
G_{D}(x, y) \simeq h(\Phi(\rho(x, y)), x, y) g_{0}(x, y) \log \left(e+\frac{\Phi(\rho(x, y))}{\Phi\left(\delta_{\vee}(x, y)\right)}\right) .
$$


Proof. Take $x, y \in D$ and let $r:=\rho(x, y)$ and $\delta_{\vee}=\delta_{\vee}(x, y)$. Using Lemma 5.1(i) (which is applicable due to (5.21)), (5.12), the second comparability in (5.15), (3.10) and the scaling property of $\Phi$, we get that, if $\delta_{\vee}>2 r$, then

$$
\int_{0}^{\Phi(r)} \frac{h(s, x, y)}{\phi(1 / s)} d s \simeq \int_{0}^{\Phi(r)} s^{\beta_{1}} h(s, x, y) d s \simeq \Phi(r)^{\gamma} h(\Phi(r), x, y)
$$

and if $\delta_{\vee} \leq 2 r$, then

$$
\begin{aligned}
& \int_{0}^{\Phi(r)} \frac{h(s, x, y)}{\phi(1 / s)} d s=\int_{0}^{2^{-1} \Phi\left(2^{-1}\left(\delta_{\vee}\right)\right)} \frac{h(s, x, y)}{\phi(1 / s)} d s+\int_{2^{-1} \Phi\left(2^{-1}(\delta \vee)\right)}^{\Phi(r)} \frac{h(s, x, y)}{\phi(1 / s)} d s \\
& \simeq \int_{0}^{2^{-1} \Phi\left(2^{-1}\left(\delta_{\vee}\right)\right)} s^{\beta_{1}} h(s, x, y) d s+\Phi(r)^{\gamma} h(\Phi(r), x, y) \int_{2^{-1} \Phi\left(2^{-1}\left(\delta_{\vee}\right)\right)}^{\Phi(r)} s^{\beta_{1}-\gamma} d s \\
& \simeq \Phi\left(\delta_{\vee}\right)^{\gamma} h\left(\Phi\left(\delta_{\vee}\right), x, y\right)+\Phi(r)^{\gamma} h(\Phi(r), x, y) \log \left(e+\frac{\Phi(r)}{\Phi\left(\delta_{\vee}\right)}\right) \\
& \simeq \Phi(r)^{\gamma} h(\Phi(r), x, y) \log \left(e+\frac{\Phi(r)}{\Phi\left(\delta_{\vee}\right)}\right) .
\end{aligned}
$$

The last comparability above is valid by the second comparability in (5.15). Therefore, in either case, it holds that

$$
\int_{0}^{\Phi(r)} \frac{h(s, x, y)}{\phi(1 / s)} d s \simeq \Phi(r)^{\gamma} h(\Phi(r), x, y) \log \left(e+\frac{\Phi(r)}{\Phi\left(\delta_{\vee}\right)}\right) .
$$

Combining this with Proposition 5.3 and Theorem 5.8, we get (5.24).

Now we also assume that $\Psi \simeq \Phi$. Then as in the proof of Theorem 5.10, one can check that in (5.24), if $\delta_{\vee}>r$, then the second term dominates the first one, and if $\delta_{\vee} \leq r$, then the first term dominates the second one. Combining with the facts that $\psi(r) \simeq \Phi(r)^{\gamma-1}$ for $0<r<\operatorname{diam}(D)$ since $\beta_{1}=\beta_{2}$ and that $g_{0}(x, y) \simeq \psi(r) / V(x, r)$ when $\delta_{\vee} \leq r$, we obtain (5.25) .

\section{Parabolic Harnack inequality and Hölder Regularity}

Throughout this section, we assume that $h(t, x, y)$ is a regular boundary function and that either $(1)$ $\left(\right.$ Poly- $\left.R_{1}\right)$ and $\mathbf{H K}_{\mathbf{B}}^{\mathbf{h}}$ hold, or (2) (Poly- $\left.\infty\right)$ and $\mathbf{H K} \mathbf{\mathbf { H }}$ hold.

For $x_{0} \in D$ and $r>0$, let $\tau_{B\left(x_{0}, r\right)}:=\inf \left\{s>0: X_{s} \notin B\left(x_{0}, r\right)\right\}$ and $X^{B\left(x_{0}, r\right)}$ be the part process of $X$ in $B\left(x_{0}, r\right)$. Denote by $q_{B\left(x_{0}, r\right)}(t, x, y)$ the heat kernel of $X^{B\left(x_{0}, r\right)}$. By the strong Markov property,

$$
q_{B\left(x_{0}, r\right)}(t, x, y)=q(t, x, y)-\mathbb{E}_{x}\left[q\left(t-\tau_{B\left(x_{0}, r\right)}, X_{\tau_{B\left(x_{0}, r\right)}}, y\right) ; \tau_{B\left(x_{0}, r\right)}<t\right] .
$$

Recall the definition of $\psi$ in (4.8).

Lemma 6.1. There exist constants $C>0$ and $\epsilon \in(0,1 / 4)$ such that for all $x_{0} \in D$ and $r \in\left(0, \delta_{D}\left(x_{0}\right)\right)$,

$$
q_{B\left(x_{0}, r\right)}(t, x, y) \geq \frac{C}{V\left(x_{0}, \psi^{-1}(t)\right)} \quad \text { for all } t \in(0, \psi(\epsilon r)] \text { and } x, y \in B\left(x_{0}, \epsilon \psi^{-1}(t)\right) .
$$

Proof. Since the proofs are similar, we only give the proof in case (1).

Fix $x_{0} \in D$ and $r \in\left(0, \delta_{D}\left(x_{0}\right)\right)$. Let $\epsilon \in(0,1 / 4)$ to be chosen later. Let $0<t \leq \psi(\epsilon r)$ and $x, y \in B\left(x_{0}, \epsilon \psi^{-1}(t)\right)$. Clearly, $x, y \in B\left(x_{0}, \epsilon^{2} r\right)$. Further, $\delta_{\wedge}(x, y)>\delta_{D}\left(x_{0}\right)-\epsilon^{2} r>r-r / \overline{4}>\psi^{-1}(t)>$ $2 \epsilon \psi^{-1}(t)>\rho(x, y)$. Therefore, we have $\delta_{\wedge}(x, y)>\psi^{-1}(t)=\rho(x, y) \vee \psi^{-1}(t)$. From Corollary 4.6(i) (with $T=\psi(\operatorname{diam}(D)))$ and (3.2), it follows that there exist constants $c_{1}, c_{2}>0$ (independent of $t, x_{0}, x, y$ ) such that

$$
q(t, x, y) \geq \frac{c_{1}}{V\left(x, \psi^{-1}(t)\right)} \geq \frac{c_{2}}{V\left(x_{0}, \psi^{-1}(t)\right)} .
$$

Let $z \in D \backslash B\left(x_{0}, r\right)$. Then since $t \leq \psi(\epsilon r)$ and $\epsilon<1 / 4$,

$$
\rho(z, y) \geq \rho\left(z, x_{0}\right)-\rho\left(y, x_{0}\right) \geq\left(1-\epsilon^{2}\right) \rho\left(z, x_{0}\right) \geq\left(1-\epsilon^{2}\right) r \geq(2 \epsilon)^{-1} \psi^{-1}(t) .
$$

Then according to Proposition 4.5(i), (3.1) and (3.2), since $h \leq 1$, there exist constants $c_{3}, c_{4}, c_{5}>0$ (independent of $\left.t, x_{0}, z, y\right)$ such that for every $0<s<t$,

$$
q(s, z, y) \leq \frac{c_{3} s}{V(z, \rho(z, y)) \psi(\rho(z, y))} \leq \frac{c_{3}}{V(z, \rho(z, y))} \leq \frac{c_{4}}{V\left(x_{0}, \rho(z, y)\right)} \leq \frac{c_{5} \epsilon^{d_{1}}}{V\left(x_{0}, \psi^{-1}(t)\right)}
$$


Combining (6.1) with (6.2) and (6.3), we get that

$$
q_{B\left(x_{0}, r\right)}(t, x, y) \geq \frac{c_{2}-c_{5} \epsilon^{d_{1}}}{V\left(x_{0}, \psi^{-1}(t)\right)} .
$$

Now we finish the proof by choosing $\epsilon=\left(c_{2} /\left(2 c_{5}\right)\right)^{1 / d_{1}}$ so that $c_{2}-c_{5} \epsilon^{d_{1}}=c_{2} / 2$.

Remark 6.2. By using (4.9) we may replace $\psi(\epsilon r)$ and $\epsilon \psi^{-1}(r)$ in the statement of Lemma 6.1 with $\epsilon \psi(r)$ and $\psi^{-1}(\epsilon r)$ respectively, cf. [19, p.3758].

Lemma 6.3. There exists a constant $C>1$ such that for all $x \in D$ and $r \in\left(0, \delta_{D}(x)\right)$,

$$
C^{-1} \psi(r) \leq \mathbb{E}^{x}\left[\tau_{B(x, r)}\right] \leq C \psi(r) .
$$

Proof. Fix $x \in D$ and $r \in\left(0, \delta_{D}(x)\right)$. Let $\epsilon \in(0,1 / 4)$ be as in the statement of Lemma 6.1. Then by Lemma 6.1, we have that

$$
q_{B(x, r)}(\psi(\epsilon r), x, y) \geq \frac{c_{1}}{V(x, \epsilon r)}, \quad y \in B\left(x, \epsilon^{2} r\right) .
$$

By (3.1), this implies that

$$
\mathbb{P}^{x}\left(\tau_{B(x, r)}>\psi(\epsilon r)\right) \geq \int_{B\left(x, \epsilon^{2} r\right)} q_{B(x, r)}(\psi(\epsilon r), x, y) d y \geq \frac{c_{1} V\left(x, \epsilon^{2} r\right)}{V(x, \epsilon r)} \geq c_{2} .
$$

Hence, by Markov's inequality and (4.9), we get that

$$
\mathbb{E}^{x}\left[\tau_{B(x, r)}\right] \geq \psi(\epsilon r) \mathbb{P}^{x}\left(\tau_{B(x, r)}>\psi(\epsilon r)\right) \geq c_{2} \psi(\epsilon r) \geq c_{3} \psi(r) .
$$

To obtain the upper bound in (6.4), we first assume that $\mathbf{H K}_{\mathbf{B}}^{\mathbf{h}}$ holds. We claim that there exists a constant $A>1$ such that

$$
\sup _{z \in B(x, r)} \mathbb{P}^{z}\left(\tau_{B(x, r)}>\psi(A r)\right) \leq \frac{1}{2} .
$$

Indeed, according to Proposition 4.5(i) and Theorem 4.7, since $h \leq 1$, there exists $c_{4}>1$ such that

$$
q(t, z, y) \leq c_{4}\left(V\left(z, \psi^{-1}(t)\right)^{-1} \mathbf{1}_{\{t \leq 1\}}+e^{-\phi\left(\lambda_{D}\right) t} \mathbf{1}_{\{t>1\}}\right), \quad z, y \in B(x, r) .
$$

Further, by (3.1), there is $c_{5}>1$ such that for all $z \in B(x, r)$,

$$
V\left(z, c_{5} r\right) \geq V\left(x,\left(c_{5}-1\right) r\right) \geq 2 c_{4} V(x, r) .
$$

Let $A>c_{5}$ be a constant such that

$$
\exp \left(\phi\left(\lambda_{D}\right) \psi\left(A c_{5}^{-1} \psi^{-1}(1)\right)\right) \geq 2 m(D) .
$$

In case when $r \leq c_{5}^{-1} \psi^{-1}(1)$, we see from (6.6) and (6.7) that for all $z \in B(x, r)$,

$$
\mathbb{P}^{z}\left(\tau_{B(x, r)}>\psi(A r)\right) \leq \mathbb{P}^{z}\left(\tau_{B(x, r)}>\psi\left(c_{5} r\right)\right) \leq \int_{B(x, r)} q\left(\psi\left(c_{5} r\right), z, y\right) d y \leq \frac{c_{4} V(x, r)}{V\left(z, c_{5} r\right)} \leq \frac{1}{2} .
$$

On the other hand, if $r>c_{5}^{-1} \psi^{-1}(1)$, then since $B(x, r) \subset B\left(x, \delta_{D}(x)\right) \subset D$, we get from (6.6) and (6.8) that for all $z \in B(x, r)$,

$$
\begin{aligned}
\mathbb{P}^{z}\left(\tau_{B(x, r)}>\psi(A r)\right) & \leq \mathbb{P}^{z}\left(\tau_{B(x, r)}>\psi\left(A c_{5}^{-1} \psi^{-1}(1)\right)\right) \leq V(x, r) \exp \left(-\phi\left(\lambda_{D}\right) \psi\left(A c_{5}^{-1} \psi^{-1}(1)\right)\right) \\
& \leq m(D) \exp \left(-\phi\left(\lambda_{D}\right) \psi\left(A c_{5}^{-1} \psi^{-1}(1)\right)\right) \leq \frac{1}{2} .
\end{aligned}
$$

Hence, (6.5) holds.

Now, by (6.5) and Markov's property, we see that for all $n \geq 2$,

$$
\begin{aligned}
& \sup _{z \in B(x, r)} \mathbb{P}^{z}\left(\tau_{B(x, r)}>n \psi(A r)\right)=\sup _{z \in B(x, r)} \mathbb{P}^{z}\left(\tau_{B(x, r)}>n \psi(A r), \tau_{B(x, r)}>\psi(A r)\right) \\
& \leq \sup _{z \in B(x, r)} \mathbb{P}^{z}\left(\mathbb{P}^{X_{\psi(A r)}}\left(\tau_{B(x, r)}>(n-1) \psi(A r)\right), \tau_{B(x, r)}>\psi(A r)\right) \\
& \leq \sup _{z \in B(x, r)} \mathbb{P}^{z}\left(\tau_{B(x, r)}>(n-1) \psi(A r)\right) \sup _{z \in B(x, r)} \mathbb{P}^{z}\left(\tau_{B(x, r)}>\psi(A r)\right)
\end{aligned}
$$




$$
\leq \cdots \leq\left(\sup _{z \in B(x, r)} \mathbb{P}^{z}\left(\tau_{B(x, r)}>\psi(A r)\right)\right)^{n} \leq 2^{-n} .
$$

Therefore, we get from (4.9) that

$$
\begin{aligned}
\mathbb{E}^{x}\left[\tau_{B(x, r)}\right] & \leq \sum_{n=1}^{\infty} n \psi(A r) \mathbb{P}^{x}\left(\tau_{B(x, r)} \in((n-1) \psi(A r), n \psi(A r)]\right) \\
& \leq c_{6} A^{\alpha_{2}\left(\beta_{2} \wedge 1\right)} \psi(r) \sum_{n=1}^{\infty} n 2^{-(n-1)}=4 c_{6} A^{\alpha_{2}\left(\beta_{2} \wedge 1\right)} \psi(r) .
\end{aligned}
$$

Similarly, by using Proposition 4.5(ii), we can obtain the upper bound in (6.4) when (Poly- $\infty$ ) and HK $_{\mathbf{U}}^{\mathrm{h}}$ hold.

Recall that the jump kernel $J(x, y)$ is given in (4.3).

Lemma 6.4. There exists a constant $C>1$ such that for all $x \in D$ and $r \in\left(0, \delta_{D}(x)\right)$,

$$
\int_{D \backslash B(x, r)} J(x, y) d y \leq \frac{C}{\psi(r)} .
$$

Proof. By Theorem 4.1 and the fact that $h \leq 1$, we have that

$$
\begin{aligned}
\int_{D \backslash B(x, r)} J(x, y) d y & \leq c_{1}\left(\int_{D \backslash B(x, r)} \frac{\int_{0}^{\Phi(\rho(x, y))} w(s) d s}{V(x, \rho(x, y)) \Psi(\rho(x, y))} d y+\int_{D \backslash B(x, r)} \frac{w(\Phi(\rho(x, y)))}{V(x, \rho(x, y))} d y\right) \\
& =: c_{1}\left(I_{1}+I_{2}\right) .
\end{aligned}
$$

By the first inequality in (2.1), since $\Psi \geq \Phi$, we see that

$$
I_{1} \leq \int_{D \backslash B(x, r)} \frac{e \Phi(\rho(x, y)) \phi(1 / \Phi(\rho(x, y)))}{V(x, \rho(x, y)) \Psi(\rho(x, y))} d y \leq \int_{D \backslash B(x, r)} \frac{e d y}{V(x, \rho(x, y)) \psi(\rho(x, y))} .
$$

It follows from (2.10) that $I_{2} \leq \frac{e}{e-2} \int_{D \backslash B(x, r)} V(x, \rho(x, y))^{-1} \psi(\rho(x, y))^{-1} d y$. Hence, by (3.1), (4.9) and the proof of [18, Lemma 2.1], we conclude that $I_{1}+I_{2} \leq c_{2} / \psi(r)$.

Let $Z:=\left(V_{s}, X_{s}\right)_{s \geq 0}$ be the time-space process corresponding to $X$, where $V_{s}=V_{0}-s$. The augmented filtration of $Z$ will be denoted by $\left(\widetilde{\mathcal{F}}_{s}\right)_{s \geq 0}$. The law of the time-space process $s \mapsto Z_{s}$ starting from $(t, x)$ will be denoted by $\mathbb{P}^{(t, x)}$. For every open subset $B$ of $[0, \infty) \times D$, define $\tau_{B}=\inf \left\{s>0: Z_{s} \notin B\right\}$ and $\sigma_{B}=\tau_{B^{c}}$.

Recall that a Borel measurable function $u:[0, \infty) \times D \rightarrow \mathbb{R}$ is parabolic (or caloric) on $(a, b) \times B\left(x_{0}, r\right)$ with respect to the process $X$ if for every relatively compact open set $U \subset(a, b) \times B\left(x_{0}, r\right)$ it holds that $u(t, x)=\mathbb{E}^{(t, x)} u\left(Z_{\tau_{U}}\right)$ for all $(t, x) \in U$.

We denote by $d t \otimes m$ the product of the Lebesgue measure on $[0, \infty)$ and $m$ on $E$.

Lemma 6.5. Let $\epsilon \in(0,1 / 4)$ be the constant from Lemma 6.1. For every $\delta \in(0, \epsilon]$, there exists a constant $C_{1}>0$ such that for all $x \in D, r \in\left(0, \delta_{D}(x)\right), t \geq \delta \psi(r)$, and any compact set $A \subset[t-$ $\delta \psi(r), t-\delta \psi(r) / 2] \times B\left(x, \psi^{-1}(\epsilon \delta \psi(r) / 2)\right)$,

$$
\mathbb{P}^{(t, x)}\left(\sigma_{A}<\tau_{[t-\delta \psi(r), t] \times B(x, r)}\right) \geq C_{1} \frac{d t \otimes m(A)}{V(x, r) \psi(r)} .
$$

Proof. Let $\tau_{r}=\tau_{[t-\delta \psi(r), t] \times B(x, r)}$ and $A_{s}=\{y \in D:(s, y) \in A\}$. For any $t, r>0$ and $x \in D$ such that $B(x, r) \subset D$,

$$
\begin{aligned}
& \delta \psi(r) \mathbb{P}^{(t, x)}\left(\sigma_{A}<\tau_{r}\right) \geq \int_{0}^{\delta \psi(r)} \mathbb{P}^{(t, x)}\left(\int_{0}^{\tau_{r}} \mathbf{1}_{A}\left(t-s, X_{s}\right) d s>0\right) d u \\
& \geq \int_{0}^{\delta \psi(r)} \mathbb{P}^{(t, x)}\left(\int_{0}^{\tau_{r}} \mathbf{1}_{A}\left(t-s, X_{s}\right) d s>u\right) d u=\mathbb{E}^{(t, x)}\left[\int_{0}^{\tau_{r}} \mathbf{1}_{A}\left(t-s, X_{s}\right) d s\right] .
\end{aligned}
$$

For any $t \geq \delta \psi(r)$,

$$
\mathbb{E}^{(t, x)}\left[\int_{0}^{\tau_{r}} \mathbf{1}_{A}\left(t-s, X_{s}\right) d s\right] \geq \int_{\delta \psi(r) / 2}^{\delta \psi(r)} \mathbb{P}^{(t, x)}\left(\left(t-s, X_{s}^{B(x, r)}\right) \in A\right) d s
$$




$$
=\int_{\delta \psi(r) / 2}^{\delta \psi(r)} \mathbb{P}^{x}\left(X_{s}^{B(x, r)} \in A_{t-s}\right) d s=\int_{\delta \psi(r) / 2}^{\delta \psi(r)} d s \int_{A_{t-s}} q_{B(x, r)}(s, x, y) d y .
$$

Let $s \in[\delta \psi(r) / 2, \delta \psi(r)]$ and $y \in B\left(x, \psi^{-1}(\epsilon \delta \psi(r) / 2)\right)$. Then $s \leq \epsilon \psi(r)$ and $\psi^{-1}(\epsilon \delta \psi(r) / 2) \leq \psi^{-1}(\epsilon s)$ so that $y \in B\left(x, \psi^{-1}(\epsilon s)\right)$. Hence, by (3.1), (4.9), Lemma 6.1 and Remark 6.2.

$$
q_{B(x, r)}(s, x, y) \geq c_{1} V\left(x, \psi^{-1}(s)\right)^{-1} \geq c_{2} V(x, r)^{-1} .
$$

Therefore

$$
\mathbb{E}^{(t, x)}\left[\int_{0}^{\tau_{r}} \mathbf{1}_{A}\left(t-s, X_{s}\right) d s\right] \geq \frac{c_{2}}{V(x, r)} \int_{\delta \psi(r) / 2}^{\delta \psi(r)} d s \int_{A_{t-s}} d y=c_{2} \frac{d t \otimes m(A)}{V(x, r)} .
$$

Combining with (6.11), we arrive at (6.10).

Theorem 6.6. There exists a constant $\eta \in(0,1]$ such that for all $\delta \in(0,1)$, there exists a constant $C=C(\delta)>0$ so that for every $x_{0} \in D, r \in\left(0, \delta_{D}\left(x_{0}\right)\right), t_{0} \geq 0$, and any function $u$ on $(0, \infty) \times D$ which is parabolic in $\left(t_{0}, t_{0}+\psi(r)\right) \times B\left(x_{0}, r\right)$ and bounded in $\left(t_{0}, t_{0}+\psi(r)\right) \times D$, we have

$$
|u(s, x)-u(t, y)| \leq C\left(\frac{\psi^{-1}(|s-t|)+\rho(x, y)}{r}\right)^{\eta} \underset{\left[t_{0}, t_{0}+\psi(r)\right] \times D}{\operatorname{essup}}|u|,
$$

for every $s, t \in\left(t_{0}+\psi(r)-\psi(\delta r), t_{0}+\psi(r)\right)$ and $x, y \in B\left(x_{0}, \delta r\right)$.

Proof. Using (3.1), (4.9) and Lemmas 6.3, 6.4 and 6.5, the result can be proved using the same argument as in the proof of [17, Theorem 4.14] (see also the proof of [19, Proposition 3.8]). We omit details here.

Lemma 6.7. Let $\epsilon \in(0,1 / 4)$ be the constant from Lemma 6.1 and let $\delta \in(0, \epsilon / 4)$ be such that $4 \delta \psi(2 r) \leq \epsilon \psi(r)$ for all $r>0$. Then there exists a constant $C_{2}>0$ such that for all $x_{0} \in D$, $R \in\left(0, \delta_{D}\left(x_{0}\right)\right), r \in\left(0, \psi^{-1}(\epsilon \delta \psi(R) / 2) / 2\right], \delta \psi(R) / 2 \leq t-s \leq 4 \delta \psi(2 R), x \in B\left(x_{0}, \psi^{-1}(\epsilon \delta \psi(R) / 2) / 2\right)$, and $z \in B\left(x_{0}, \psi^{-1}(\epsilon \delta \psi(R) / 2)\right)$,

$$
\mathbb{P}^{(t, z)}\left(\sigma_{\{s\} \times B(x, r)} \leq \tau_{[s, t] \times B\left(x_{0}, R\right)}\right) \geq C_{2} \frac{V(x, r)}{V(x, R)} .
$$

Proof. The left-hand side of (6.13) is equal to

$$
\mathbb{P}^{z}\left(X_{t-s}^{B\left(x_{0}, R\right)} \in B(x, r)\right)=\int_{B(x, r)} q_{B\left(x_{0}, R\right)}(t-s, z, y) d y .
$$

Note that $t-s \leq 4 \delta \psi(2 R) \leq \epsilon \psi(R)$ by the choice of $\delta$. Next, if $z \in B(x, r)$, then $\rho\left(z, x_{0}\right) \leq \rho(z, x)+$ $\rho\left(x, x_{0}\right) \leq r+\rho\left(x, x_{0}\right) \leq \psi^{-1}(\epsilon \delta \psi(R) / 2) \leq \psi^{-1}(\epsilon(t-s))$, implying that $z \in B\left(x_{0}, \psi^{-1}(\epsilon(t-s))\right)$ (with the same conclusion for $y \in B(x, r))$. Thus it follows from Lemma 6.1, Remark 6.2 and (3.2) that $q_{B\left(x_{0}, R\right)}(t-s, z, y) \geq c_{1} V\left(x_{0}, R\right)^{-1} \geq c_{2} V(x, R)^{-1}$. By inserting in (6.14) we obtain (6.13).

In the remainder of this section, we further assume that $h$ is of Harnack-type.

Suppose that $x, y, z \in D$ are such that $\rho(x, z) \leq \rho(x, y) / 2$. Then

$$
\frac{2}{3} \rho(x, y) \leq \rho(z, y) \leq \frac{3}{2} \rho(x, y) .
$$

As a consequence, by the scalings of $\Phi$ and $\Psi$, there exists $a>1$ such that

$$
a^{-1} \Phi(\rho(x, y)) \leq \Phi(\rho(z, y)) \leq a \Phi(\rho(x, y)), \quad a^{-1} \Psi(\rho(x, y)) \leq \Psi(\rho(z, y)) \leq a \Psi(\rho(x, y)) .
$$

Proposition 6.8. Suppose that $h$ is of Harnack-type. Then there exists a constant $C>0$ such that for all $x, y, z \in D$ satisfying $\rho(x, z) \leq\left(\rho(x, y) \wedge \delta_{D}(x)\right) / 2$, it holds that

$$
J(x, y) \leq C J(z, y) .
$$

Proof. This follows from Theorem 4.1, (3.7), the scaling property of $w,(3.10)$ and (6.16). 
Corollary 6.9. Suppose that $h$ is of Harnack-type. Then there exists a constant $C>0$ such that for all $x, y \in D$ and $0<r \leq\left(\rho(x, y) \wedge \delta_{D}(x)\right) / 2$, it holds that

$$
J(x, y) \leq \frac{C}{V(x, r)} \int_{B(x, r)} J(z, y) d z .
$$

Proof. Let $x, y \in D$ and $r>0$ be as in the statement. If $z \in B(x, r)$, then $\rho(x, z)<r \leq \rho(x, y) / 2$. Therefore, by Proposition 6.8, $J(x, y) \leq c_{1} J(z, y)$, whence the claim immediately follows.

Lemma 6.10. Suppose that $h$ is of Harnack-type. Let $\epsilon \in(0,1 / 4)$ be the constant from Lemma 6.1, and $\theta \geq 1 / 2$. Further, let $0<\delta_{0}<\epsilon$, and $0<\delta_{1}<\delta_{2}<\delta_{3}<\delta_{4}$ be such that $\left(\delta_{3}-\delta_{2}\right) \psi(r) \geq \psi\left(\delta_{0} r\right)$ and $\delta_{4} \psi(r) \leq \psi(\epsilon r)$ for all $r \in(0, \operatorname{diam}(D))$. For $x_{0} \in D, t_{0} \geq 0$ and $r \in\left(0, \delta_{D}(x)\right)$, define

$$
\begin{array}{ll}
Q_{1}=\left(t_{0}, t_{0}+\delta_{4} \psi(r)\right) \times B\left(x_{0}, \delta_{0}^{2} r\right), & Q_{2}=\left(t_{0}, t_{0}+\delta_{4} \psi(r)\right) \times B\left(x_{0}, r\right), \\
Q_{3}=\left[t_{0}+\delta_{1} \psi(r), t_{0}+\delta_{2} \psi(r)\right] \times B\left(x_{0}, \delta_{0}^{2} r / 2\right), & Q_{4}=\left[t_{0}+\delta_{3} \psi(r), t_{0}+\delta_{4} \psi(r)\right] \times B\left(x_{0}, \delta_{0}^{2} r / 2\right) .
\end{array}
$$

Let $f:\left(t_{0}, \infty\right) \times D \rightarrow[0, \infty)$ be bounded and supported in $\left(t_{0}, \infty\right) \times\left(D \backslash B\left(x_{0},(1+\theta) r\right)\right)$. Then there exists a constant $C_{2}>0$ such that

$$
\mathbb{E}^{\left(t_{1}, y_{1}\right)} f\left(Z_{\tau_{Q_{1}}}\right) \leq C_{2} \mathbb{E}^{\left(t_{2}, y_{2}\right)} f\left(Z_{\tau_{Q_{2}}}\right) \quad \text { for all }\left(t_{1}, y_{1}\right) \in Q_{3},\left(t_{2}, y_{2}\right) \in Q_{4} .
$$

Proof. Without loss of generality we may assume that $t_{0}=0$. Fix $x_{0} \in D$. For $s>0$, we set $B_{s}=B\left(x_{0}, s\right)$. Let $\left(t_{1}, y_{1}\right) \in Q_{3}$ and $\left(t_{2}, y_{2}\right) \in Q_{4}$. By the Lévy system formula for the time-space process $Z$ we have

$$
\begin{aligned}
\mathbb{E}^{\left(t_{2}, y_{2}\right)} f\left(Z_{\tau_{Q_{2}}}\right)=\mathbb{E}^{\left(t_{2}, y_{2}\right)} f\left(t_{2}-\left(\tau_{B_{r}} \wedge t_{2}\right), X_{\tau_{B_{r}} \wedge t_{2}}\right) \\
=\mathbb{E}^{\left(t_{2}, y_{2}\right)}\left[\int_{0}^{t_{2}} \mathbf{1}_{t \leq \tau_{B_{r}}} d t \int_{D \backslash B\left(x_{0},(1+\theta) r\right)} f\left(t_{2}-t, v\right) J\left(X_{t}, v\right) d v\right] \\
=\int_{0}^{t_{2}} d t \int_{D \backslash B\left(x_{0},(1+\theta) r\right)} f\left(t_{2}-t, v\right) \mathbb{E}^{\left(t_{2}, y_{2}\right)}\left[\mathbf{1}_{t \leq \tau_{B_{r}}} J\left(X_{t}, v\right)\right] d v \\
=\int_{0}^{t_{2}} d s \int_{D \backslash B\left(x_{0},(1+\theta) r\right)} f(s, v) \mathbb{E}^{\left(t_{2}, y_{2}\right)}\left[\mathbf{1}_{t_{2}-s \leq \tau_{B_{r}}} J\left(X_{t_{2}-s}, v\right)\right] d v \\
=\int_{0}^{t_{2}} d s \int_{D \backslash B\left(x_{0},(1+\theta) r\right)} f(s, v) d v \int_{B_{r}} q_{B_{r}}\left(t_{2}-s, y_{2}, z\right) J(z, v) d z \\
\geq \int_{0}^{t_{1}} d s \int_{D \backslash B\left(x_{0},(1+\theta) r\right)} f(s, v) d v \int_{B_{\delta_{0}^{2} r}} q_{B_{r}}\left(t_{2}-s, y_{2}, z\right) J(z, v) d z .
\end{aligned}
$$

For $s \in\left[0, t_{1}\right]$ it holds that $\psi\left(\delta_{0} r\right) \leq\left(\delta_{3}-\delta_{2}\right) \psi(r) \leq t_{2}-t_{1} \leq t_{2}-s \leq \delta_{4} \psi(r) \leq \psi(\epsilon r)$, hence $\delta_{0}^{2} r \leq \epsilon \delta_{0} r \leq \epsilon \psi^{-1}\left(t_{2}-s\right)$. Therefore, for any $z \in B_{\delta_{0}^{2} r}$, by Lemma 6.1 and (3.2),$q_{B_{r}}\left(t_{2}-s, y_{2}, z\right) \geq$ $c_{1} V\left(x_{0}, \psi^{-1}\left(t_{2}-s\right)\right)^{-1} \geq c_{1} V\left(x_{0}, \epsilon r\right)^{-1}$. We conclude that

$$
\mathbb{E}^{\left(t_{2}, y_{2}\right)} f\left(Z_{\tau_{Q_{2}}}\right) \geq \frac{c_{1}}{V\left(x_{0}, \epsilon r\right)} \int_{0}^{t_{1}} d s \int_{D \backslash B\left(x_{0},(1+\theta) r\right)} f(s, v) d v \int_{B_{\delta_{0}^{2} r}} J(z, v) d z .
$$

Now, by using the Lévy system formula again, similar to (6.17) we obtain that

$$
\begin{aligned}
\mathbb{E}^{\left(t_{1}, y_{1}\right)} f\left(Z_{\tau_{Q_{1}}}\right)=\int_{0}^{t_{1}} d s \int_{D \backslash B\left(x_{0},(1+\theta) r\right)} f(s, v) d v \int_{B_{\delta_{0}^{2} r}} q_{B_{\delta_{0}^{2}} r}\left(t_{1}-s, y_{1}, z\right) J(z, v) d z \\
=\int_{0}^{t_{1}} d s \int_{B_{\delta_{0}^{2} r}} q_{B_{\delta_{0}^{2}}^{2}}\left(t_{1}-s, y_{1}, z\right) d z \int_{D \backslash B\left(x_{0},(1+\theta) r\right)} f(s, v) J(z, v) d v \\
=\int_{0}^{t_{1}} d s\left(\int_{B_{\delta_{0}^{2} r} \backslash B_{3 \delta_{0}^{2} r / 4}} q_{B_{\delta_{0}^{2} r}}\left(t_{1}-s, y_{1}, z\right) d z \int_{D \backslash B\left(x_{0},(1+\theta) r\right)} f(s, v) J(z, v) d v\right.
\end{aligned}
$$




$$
\left.+\int_{B_{3 \delta_{0}^{2} r / 4}} q_{B_{\delta_{0}^{2} r}}\left(t_{1}-s, y_{1}, z\right) d z \int_{D \backslash B\left(x_{0},(1+\theta) r\right)} f(s, v) J(z, v) d v\right)=: \int_{0}^{t_{1}} d s\left(I_{1}+I_{2}\right) .
$$

Let $z \in B_{\delta_{0}^{2} r} \backslash B_{3 \delta_{0}^{2} r / 4}$. Since $y_{1} \in B_{\delta_{0}^{2} r / 2}$, we have that $2 \rho\left(x_{0}, y_{1}\right) \leq \delta_{0}^{2} r / 4 \leq \rho\left(y_{1}, z\right) \leq 3 \delta_{0}^{2} r / 2$, which implies by (4.9) that $\psi\left(\rho\left(y_{1}, z\right)\right) \geq c_{2} \psi(r) \geq c_{2} \delta_{2}^{-1} t_{1}$. Hence by Proposition 4.5, (3.1) and (3.2), we get that for any $s>0$,

$$
\begin{aligned}
q_{B_{\delta_{0}^{2} r}}\left(t_{1}-s, y_{1}, z\right) & \leq q\left(t_{1}-s, y_{1}, z\right) \leq \frac{c_{3} t_{1}}{V\left(y_{1}, \rho\left(y_{1}, z\right)\right) \psi\left(\rho\left(y_{1}, z\right)\right)} \leq \frac{c_{2}^{-1} c_{3} \delta_{2}}{V\left(y_{1}, \rho\left(y_{1}, z\right)\right)} \\
& \leq \frac{c_{4}}{V\left(x_{0}, \rho\left(y_{1}, z\right)\right)} \leq \frac{c_{4}}{V\left(x_{0}, \delta_{0}^{2} r / 4\right)} \leq \frac{c_{5}}{V\left(x_{0}, \epsilon r\right)} .
\end{aligned}
$$

Therefore,

$$
I_{1} \leq \frac{c_{5}}{V\left(x_{0}, \epsilon r\right)} \int_{B_{\delta_{0}^{2} r} \backslash B_{3 \delta_{0}^{2} r / 4}} d z \int_{D \backslash B\left(x_{0},(1+\theta) r\right)} f(s, v) J(z, v) d v .
$$

Let $z \in B_{3 \delta_{0}^{2} r / 4}$. Then by (3.2), we have

$$
V\left(z, \delta_{0}^{2} r / 4\right) \geq c_{6} V\left(x_{0}, \epsilon r\right) .
$$

We also have that $\delta_{D}(z) \geq r-3 \delta_{0}^{2} r / 4 \geq\left(1-\delta_{0}^{2}\right) r$, and for $v \in D \backslash B\left(x_{0},(1+\theta) r\right)$,

$$
\rho(z, v) \geq \rho\left(x_{0}, v\right)-\rho\left(x_{0}, z\right) \geq(1+\theta) r-\frac{3 \delta_{0}^{2} r}{4} \geq \frac{3 r}{2}-\frac{3 \delta_{0}^{2} r}{4} \geq\left(1-\delta_{0}^{2}\right) r .
$$

Thus, for any $w \in B\left(z, \delta_{0}^{2} r / 4\right)$, since $\delta_{0}^{2}<2 / 5$,

$$
\frac{1}{2}\left(\rho(z, v) \wedge \delta_{D}(z)\right) \geq \frac{1}{2}\left(1-\delta_{0}^{2}\right) r \geq \frac{\delta_{0}^{2} r}{4} \geq \rho(z, w) .
$$

From Proposition 6.8, we get that $J(z, v) \leq c_{7} J(w, v)$. Therefore, by (6.19),

$$
\begin{aligned}
I_{2} & =\int_{B_{3 \delta_{0}^{2} r / 4}} q_{B_{\delta_{0}^{2}}^{2}}\left(t_{1}-s, y_{1}, z\right) d z \int_{D \backslash B\left(x_{0},(1+\theta) r\right)} f(s, v) J(z, v) d v \\
& \leq \int_{B_{3 \delta_{0}^{2} r / 4}} q_{B_{\delta_{0}^{2}}^{2} r}\left(t_{1}-s, y_{1}, z\right) d z\left(\frac{c_{7}}{V\left(z, \delta_{0}^{2} r / 4\right)} \int_{B\left(z, \delta_{0}^{2} r / 4\right)} J(w, v) d w \int_{D \backslash B\left(x_{0},(1+\theta) r\right)} f(s, v) d v\right) \\
& \leq \frac{c_{6}^{-1} c_{7}}{V\left(x_{0}, \epsilon r\right)} \int_{B\left(z, \delta_{0}^{2} r / 4\right)} d w \int_{D \backslash B\left(x_{0},(1+\theta) r\right)} f(s, v) J(w, v) d v .
\end{aligned}
$$

It follows that

$$
\begin{aligned}
\mathbb{E}^{\left(t_{1}, y_{1}\right)} f\left(Z_{\tau_{Q_{1}}}\right) & =\int_{0}^{t_{1}} d s\left(I_{1}+I_{2}\right) \leq \frac{c_{8}}{V\left(x_{0}, \epsilon r\right)} \int_{0}^{t_{1}} d s \int_{B_{\delta_{0}^{2} r}} d z \int_{D \backslash B\left(x_{0},(1+\theta) r\right)} f(s, v) J(z, v) d v \\
& =\frac{c_{8}}{V\left(x_{0}, \epsilon r\right)} \int_{0}^{t_{1}} d s \int_{D \backslash B\left(x_{0},(1+\theta) r\right)} f(s, v) d v \int_{B_{\delta_{0}^{2} r}} J(z, v) d z \stackrel{\frac{6.18}{\leq}}{\leq} c_{9} \mathbb{E}^{\left(t_{2}, y_{2}\right)} f\left(Z_{\tau_{Q_{2}}}\right) .
\end{aligned}
$$

Theorem 6.11. Suppose that $h$ is of Harnack-type. Then there exist constants $\delta>0, C>1$ and $K \geq 1$ such that for all $t_{0} \geq 0, x_{0} \in D$ and $R \in\left(0, R_{1}\right)$ with $B\left(x_{0}, C R\right) \subset D$, and any non-negative function $u$ on $(0, \infty) \times D$ which is parabolic on $Q:=\left(t_{0}, t_{0}+4 \delta \psi(C R)\right) \times B\left(x_{0}, C R\right)$, we have

$$
\sup _{\left(t_{1}, y_{1}\right) \in Q_{-}} u\left(t_{1}, y_{1}\right) \leq K \inf _{\left(t_{2}, y_{2}\right) \in Q_{+}} u\left(t_{2}, y_{2}\right)
$$

where $Q_{-}=\left[t_{0}+\delta \psi(C R), t_{0}+2 \delta \psi(C R)\right] \times B\left(x_{0}, R\right)$ and $Q_{-}=\left[t_{0}+3 \delta \psi(C R), t_{0}+4 \delta \psi(C R)\right] \times B\left(x_{0}, R\right)$.

Proof. Using (3.1), (4.9), Lemmas 6.1, 6.3, 6.5, 6.7, 6.10 and Corollary 6.9, the result can be proved using the same argument as in the proof of [16, Lemma 5.3] (see also the proof of [19, Lemma 4.1]). We omit details here. 


\section{EXAMPLES}

Recall the definitions of $h_{p, q}(t, x, y)$ from (3.8), and $\mathcal{B}_{h}(t, x, y)$ from (4.2). We remind the reader that $h_{p, q}(t, x, y)$ is quite typical and it is the most important boundary function. Recall that $\psi(r)=$ $1 / \phi(1 / \Phi(r)), \delta_{\vee}(x, y)=\delta_{D}(x) \vee \delta_{D}(y)$ and $\delta_{\wedge}(x, y)=\delta_{D}(x) \wedge \delta_{D}(y)$. For simplicity, we will use $\delta(x)$ and $\delta(y)$ instead of $\delta_{D}(x)$ and $\delta_{D}(y)$, respectively.

We let

$$
\begin{aligned}
\delta^{t}(x) & :=\delta_{D}(x) \vee \psi^{-1}(t), \\
\delta_{\vee}^{t}(x, y) & :=\delta^{t}(x) \vee \delta^{t}(y)=\delta_{\vee}(x, y) \vee \psi^{-1}(t), \\
\delta_{\wedge}^{t}(x, y) & :=\delta^{t}(x) \wedge \delta^{t}(y)=\delta_{\wedge}(x, y) \vee \psi^{-1}(t) .
\end{aligned}
$$

The following lemma provides a list of estimates of $\mathcal{B}_{h_{p, q}}(t, x, y)$ depending on the relationship between the parameters $p, q, \beta_{1}, \beta_{2}$. The list is not exhaustive, but it suffices for our purpose. The proof of the lemma is rather technical and consists of carefully estimating the integral defining $\mathcal{B}_{h_{p, q}}(t, x, y)$. The factorization (7.2) below is inspired by (4.11) and (4.18). See also (7.15) below.

Lemma 7.1. Let $q \geq p \geq 0, p+q>0$. Suppose that (Poly- $\left.R_{1}\right)$ holds with $\beta_{1}, \beta_{2} \in(0,1)$. Then

$$
\mathcal{B}_{h_{p, q}}(t, x, y)=\frac{\Phi(\rho(x, y))}{\psi(\rho(x, y))}\left(1 \wedge \frac{\Phi\left(\delta_{D}(x)\right)}{\phi^{-1}(1 / t)^{-1}}\right)^{p}\left(1 \wedge \frac{\Phi\left(\delta_{D}(y)\right)}{\phi^{-1}(1 / t)^{-1}}\right)^{q} A_{p, q}(t, x, y),
$$

where $A_{p, q}(t, x, y)$ satisfies the following estimates for all $x, y \in D$ and $0<t \leq \psi(\rho(x, y))$ such that $\Phi(\rho(x, y))<R_{1} / 8$.

(i) If $\beta_{2}<1-p-q$, then

$$
A_{p, q}(t, x, y) \simeq\left(1 \wedge \frac{\Phi\left(\delta^{t}(y)\right)}{\Phi(\rho(x, y))}\right)^{q-p}\left(1 \wedge \frac{\Phi\left(\delta_{\wedge}^{t}(x, y)\right)}{\Phi(\rho(x, y))}\right)^{p}\left(1 \wedge \frac{\Phi\left(\delta_{\vee}^{t}(x, y)\right)}{\Phi(\rho(x, y))}\right)^{p} .
$$

(ii) If $1-p-q<\beta_{1} \leq \beta_{2}<1-q$, then

$$
A_{p, q}(t, x, y) \simeq\left(1 \wedge \frac{\Phi\left(\delta^{t}(y)\right)}{\Phi(\rho(x, y))}\right)^{q-p}\left(1 \wedge \frac{\Phi\left(\delta_{\wedge}^{t}(x, y)\right)}{\Phi(\rho(x, y))}\right)^{p}\left(1 \wedge \frac{\Phi\left(\delta_{\vee}^{t}(x, y)\right)}{\Phi(\rho(x, y))}\right)^{1-q}\left(1 \wedge \frac{\psi\left(\delta_{\vee}^{t}(x, y)\right)}{\psi(\rho(x, y))}\right)^{-1} .
$$

(iii) If $1-q<\beta_{1} \leq \beta_{2}<1-p$, then

$$
A_{p, q}(t, x, y) \simeq\left(1 \wedge \frac{\Phi\left(\delta^{t}(y)\right)}{\Phi(\rho(x, y))}\right)^{1-p}\left(1 \wedge \frac{\Phi\left(\delta_{\wedge}^{t}(x, y)\right)}{\Phi(\rho(x, y))}\right)^{p}\left(1 \wedge \frac{\psi\left(\delta^{t}(y)\right)}{\psi(\rho(x, y))}\right)^{-1} .
$$

(iv) If $1-p<\beta_{1} \leq \beta_{2}<1$, then

$$
A_{p, q}(t, x, y) \simeq\left(1 \wedge \frac{\Phi\left(\delta_{\wedge}^{t}(x, y)\right)}{\Phi(\rho(x, y))}\right)\left(1 \wedge \frac{\psi\left(\delta_{\wedge}^{t}(x, y)\right)}{\psi(\rho(x, y))}\right)^{-1} .
$$

(v) If $\beta_{1}=\beta_{2}=1-p-q$ and $p>0$, then

$$
A_{p, q}(t, x, y) \simeq\left(1 \wedge \frac{\Phi\left(\delta^{t}(y)\right)}{\Phi(\rho(x, y))}\right)^{q-p}\left(1 \wedge \frac{\Phi\left(\delta_{\wedge}^{t}(x, y)\right)}{\Phi(\rho(x, y))}\right)^{p}\left(1 \wedge \frac{\Phi\left(\delta_{\vee}^{t}(x, y)\right)}{\Phi(\rho(x, y))}\right)^{p} \log \left(e+\frac{\Phi(\rho(x, y))}{\Phi\left(\delta_{\vee}^{t}(x, y)\right)}\right) .
$$

(vi) If $\beta_{1}=\beta_{2}=1-p-q$ and $p=0$, then

$$
A_{p, q}(t, x, y) \simeq\left(1 \wedge \frac{\Phi\left(\delta^{t}(y)\right)}{\Phi(\rho(x, y))}\right)^{q} \log \left(e+\frac{\Phi(\rho(x, y))}{\Phi\left(\delta^{t}(y)\right)}\right) .
$$

(vii) If $\beta_{1}=\beta_{2}=1-q$ and $q=p$, then

$$
A_{p, q}(t, x, y) \simeq\left(1 \wedge \frac{\Phi\left(\delta_{\wedge}^{t}(x, y)\right)}{\Phi(\rho(x, y))}\right)^{p} \log \left(e+\frac{\Phi(\rho(x, y)) \wedge \Phi\left(\delta_{\vee}^{t}(x, y)\right)}{\Phi\left(\delta_{\wedge}^{t}(x, y)\right)}\right) .
$$

(viii) If $\beta_{1}=\beta_{2}=1-q$ and $q>p>0$, then

$$
A_{p, q}(t, x, y) \simeq\left(1 \wedge \frac{\Phi\left(\delta^{t}(y)\right)}{\Phi(\rho(x, y))}\right)^{q-p}\left(1 \wedge \frac{\Phi\left(\delta_{\wedge}^{t}(x, y)\right)}{\Phi(\rho(x, y))}\right)^{p} \log \left(e+\frac{\Phi(\rho(x, y)) \wedge \Phi\left(\delta^{t}(x)\right)}{\Phi\left(\delta^{t}(y)\right)}\right) .
$$

(ix) If $\beta_{1}=\beta_{2}=1-p$ and $q>p$, then

$$
A_{p, q}(t, x, y) \simeq\left(1 \wedge \frac{\Phi\left(\delta_{\wedge}^{t}(x, y)\right)}{\Phi(\rho(x, y))}\right)^{p} \log \left(e+\frac{\Phi(\rho(x, y)) \wedge \Phi\left(\delta^{t}(y)\right)}{\Phi\left(\delta^{t}(x)\right)}\right) .
$$


Proof. Fix $x, y \in D$ such that $r:=\rho(x, y)<\Phi^{-1}\left(R_{1} / 8\right)$, and $t \in(0, \psi(r)]$. Let $\delta_{\wedge}:=\delta_{\wedge}(x, y)$ and $\delta_{\vee}:=\delta_{\vee}(x, y)$, and note that $\delta_{\vee} \leq r+\delta_{\wedge}$. We also note that since $\beta_{2}<1$, by 47, Lemma 2.6, Proposition $2.9]$ and (2.2),

$$
w(s) \simeq \phi(1 / s), \quad s \in\left(0, R_{1} / 2\right),
$$

which is equivalent to that $w(\Phi(s)) \simeq \psi(s)^{-1}$ for $s \in\left(0, \Phi^{-1}\left(R_{1} / 2\right)\right)$.

Define

$$
A_{p, q}(t, x, y):=\frac{\psi(r)}{\Phi(r)} \frac{\mathcal{B}_{h_{p, q}}(t, x, y)}{h_{p, q}\left(\phi^{-1}(1 / t)^{-1}, x, y\right)} .
$$

Observe that for any $s>\phi^{-1}(1 / t)^{-1}$,

$$
\begin{aligned}
\frac{h_{p, q}(s, x, y)}{h_{p, q}\left(\phi^{-1}(1 / t)^{-1}, x, y\right)} & =\left(1 \wedge \frac{\Phi\left(\delta^{t}(x)\right)}{s}\right)^{p}\left(1 \wedge \frac{\Phi\left(\delta^{t}(y)\right)}{s}\right)^{q} \\
& =\left(1 \wedge \frac{\Phi\left(\delta^{t}(y)\right)}{s}\right)^{q-p}\left(1 \wedge \frac{\Phi\left(\delta_{\wedge}^{t}(x, y)\right)}{s}\right)^{p}\left(1 \wedge \frac{\Phi\left(\delta_{\vee}^{t}(x, y)\right)}{s}\right)^{p} .
\end{aligned}
$$

Hence, by the definition of $\mathcal{B}_{h_{p, q}}(t, x, y)$ and (7.4), we get

$$
A_{p, q}(t, x, y)=\frac{\psi(r)}{\Phi(r)} \int_{2 \phi^{-1}(1 / t)^{-1}}^{4 \Phi(r)}\left(1 \wedge \frac{\Phi\left(\delta^{t}(y)\right)}{s}\right)^{q-p}\left(1 \wedge \frac{\Phi\left(\delta_{\wedge}^{t}(x, y)\right)}{s}\right)^{p}\left(1 \wedge \frac{\Phi\left(\delta_{\vee}^{t}(x, y)\right)}{s}\right)^{p} w(s) d s .
$$

If $\delta_{\vee} \geq 2 r$, then $\delta_{\wedge} \geq \delta_{\vee}-r \geq r$ and hence we get from Lemma $5.1(\mathrm{i}),\left(\right.$ Poly- $R_{1}$ ) (by using $\beta_{2}<1$ ) and (17.3) that

$$
A_{p, q}(t, x, y) \simeq \frac{\psi(r)}{\Phi(r)} \int_{2 \phi^{-1}(1 / t)^{-1}}^{4 \Phi(r)} w(s) d s \simeq \psi(r) w(4 \Phi(r)) \simeq 1 .
$$

On the other hand, we see that in every of the cases (i)-(ix), the right-hand side of comparability relation for $A_{p, q}(t, x, y)$ is comparable to 1 . Hence the assertion of the lemma is valid when $\delta_{\vee} \geq 2 r$.

Suppose now that $\delta_{\vee}<2 r$. Since $\psi^{-1}(t)<r$, we get $\delta_{\vee}^{t}(x, y)<2 r$, hence by the scaling property (3.4) of $\Phi$, we have

$$
3 \Phi(a) \wedge 4 \Phi(r) \simeq \Phi(a) \text { for } a \in\left\{\delta^{t}(y), \delta_{\wedge}^{t}(x, y), \delta_{\vee}^{t}(x, y)\right\}
$$

(i) The desired comparability follows from Lemma 5.1(i), (Poly- $\left.R_{1}\right)$ and (7.3).

(ii) By Lemma 5.1(i)-(ii), (Poly- $\left.R_{1}\right)$ and (7.3), since $q-p+p+\beta_{2}<1<p+q+\beta_{1}$,

$$
\begin{aligned}
A_{p, q}(t, x, y) \simeq & \frac{\psi(r)}{\Phi(r)} \int_{2 \phi^{-1}(1 / t)^{-1}}^{3 \Phi\left(\delta_{\vee}^{t}(x, y)\right) \wedge 4 \Phi(r)}\left(1 \wedge \frac{\Phi\left(\delta^{t}(y)\right)}{s}\right)^{q-p}\left(1 \wedge \frac{\Phi\left(\delta_{\wedge}^{t}(x, y)\right)}{s}\right)^{p} w(s) d s \\
& +\frac{\psi(r)}{\Phi(r)} \Phi\left(\delta^{t}(y)\right)^{q-p} \Phi\left(\delta_{\wedge}^{t}(x, y)\right)^{p} \Phi\left(\delta_{\vee}^{t}(x, y)\right)^{p} \int_{3 \Phi\left(\delta_{\vee}^{t}(x, y)\right) \wedge 4 \Phi(r)}^{4 \Phi(r)} s^{-p-q} w(s) d s \\
\simeq & \frac{\psi(r)}{\Phi(r)}\left(1 \wedge \frac{\Phi\left(\delta^{t}(y)\right)}{\Phi\left(\delta_{\vee}^{t}(x, y)\right)}\right)^{q-p}\left(1 \wedge \frac{\Phi\left(\delta_{\wedge}^{t}(x, y)\right)}{\Phi\left(\delta_{\vee}^{t}(x, y)\right)}\right)^{p} \Phi\left(\delta_{\vee}^{t}(x, y)\right) w\left(\Phi\left(\delta_{\vee}^{t}(x, y)\right)\right) \\
\simeq & \frac{\Phi\left(\delta^{t}(y)\right)^{q-p} \Phi\left(\delta_{\wedge}^{t}(x, y)\right)^{p}}{\Phi(r)^{q}}\left(\frac{\Phi(r)}{\Phi\left(\delta_{\vee}^{t}(x, y)\right)}\right)^{q} \frac{\Phi\left(\delta_{\vee}^{t}(x, y)\right)}{\psi\left(\delta_{\vee}^{t}(x, y)\right)} \frac{\psi(r)}{\Phi(r)} .
\end{aligned}
$$

We used (7.6) in the second comparability above. Since $\Phi$ and $\psi$ are increasing and satisfy scaling properties, the desired comparability holds.

(iii) If $\delta_{\vee}^{t}(x, y)=\delta^{t}(y)$, then (7.7) holds by Lemma [5.1(i)-(ii), (Poly- $\left.R_{1}\right)$, (7.3) and (7.6) since $p+\beta_{2}<$ $1<p+q+\beta_{1}$. The desired comparability then follows immediately from (7.7). If $\delta_{\wedge}^{t}(x, y)=\delta^{t}(y)$, then we get, by Lemma 5.1(i)-(ii), (Poly- $\left.R_{1}\right)$, (7.3), (77.6) and the assumption $q+\beta_{1}>1$, that

$$
\begin{aligned}
A_{p, q}(t, x, y) & \simeq \frac{\psi(r)}{\Phi(r)} \int_{2 \phi^{-1}(1 / t)^{-1}}^{3 \Phi\left(\delta^{t}(y)\right) \wedge 4 \Phi(r)} w(s) d s+\frac{\psi(r)}{\Phi(r)} \Phi\left(\delta^{t}(y)\right)^{q} \int_{3 \Phi\left(\delta^{t}(y)\right) \wedge 4 \Phi(r)}^{4 \Phi(r)}\left(1 \wedge \frac{\Phi\left(\delta_{\vee}^{t}(x, y)\right)}{s}\right)^{p} s^{-q} w(s) d s \\
& \simeq \frac{\Phi\left(\delta^{t}(y)\right)}{\psi\left(\delta^{t}(y)\right)} \frac{\psi(r)}{\Phi(r)}=\left(\frac{\Phi\left(\delta^{t}(y)\right)}{\Phi(r)}\right)^{1-p}\left(\frac{\Phi\left(\delta_{\wedge}^{t}(x, y)\right)}{\Phi(r)}\right)^{p} \frac{\psi(r)}{\psi\left(\delta^{t}(y)\right)} .
\end{aligned}
$$

(iv) Since $p+\beta_{1}>1$, we get from Lemma 5.1(i)-(ii), (Poly- $\left.R_{1}\right)$, (17.3) and (17.6) that

$$
A_{p, q}(t, x, y) \simeq \frac{\psi(r)}{\Phi(r)} \int_{2 \phi^{-1}(1 / t)^{-1}}^{3 \Phi\left(\delta_{\wedge}^{t}(x, y)\right) \wedge 4 \Phi(r)} w(s) d s
$$




$$
\begin{aligned}
& +\frac{\psi(r)}{\Phi(r)} \Phi\left(\delta_{\wedge}^{t}(x, y)\right)^{p} \int_{3 \Phi\left(\delta_{\wedge}^{t}(x, y)\right) \wedge 4 \Phi(r)}^{4 \Phi(r)}\left(1 \wedge \frac{\Phi\left(\delta^{t}(y)\right)}{s}\right)^{q-p}\left(1 \wedge \frac{\Phi\left(\delta_{\vee}^{t}(x, y)\right)}{s}\right)^{p} s^{-p} w(s) d s \\
\simeq & \frac{\Phi\left(\delta_{\wedge}^{t}(x, y)\right)}{\psi\left(\delta_{\wedge}^{t}(x, y)\right)} \frac{\psi(r)}{\Phi(r)} .
\end{aligned}
$$

(v) Note that $w(s) \simeq s^{p+q-1}$ and $\psi(s) \simeq \Phi(s)^{1-p-q}$ for $s \in\left(0, R_{1}\right)$ in this case. Thus, we get from Lemma 5.1(i) and (7.6) that

$$
\begin{aligned}
A_{p, q}(t, x, y) \simeq & \frac{\psi(r)}{\Phi(r)} \int_{2 \phi^{-1}(1 / t)^{-1}}^{3 \Phi\left(\delta_{\vee}^{t}(x, y)\right) \wedge 4 \Phi(r)}\left(s \wedge \Phi\left(\delta^{t}(y)\right)\right)^{q-p}\left(s \wedge \Phi\left(\delta_{\wedge}^{t}(x, y)\right)\right)^{p} s^{p-1} d s \\
& +\frac{\psi(r)}{\Phi(r)} \Phi\left(\delta^{t}(y)\right)^{q-p} \Phi\left(\delta_{\wedge}^{t}(x, y)\right)^{p} \Phi\left(\delta_{\vee}^{t}(x, y)\right)^{p} \int_{3 \Phi\left(\delta_{\vee}^{t}(x, y)\right) \wedge 4 \Phi(r)}^{4 \Phi(r)} s^{-1} d s \\
\simeq & \frac{\Phi\left(\delta^{t}(y)\right)^{q-p} \Phi\left(\delta_{\wedge}^{t}(x, y)\right)^{p} \Phi\left(\delta_{\vee}^{t}(x, y)\right)^{p}}{\Phi(r)^{p+q}} \log \left(e+\frac{\Phi(r)}{\Phi\left(\delta_{\vee}^{t}(x, y)\right)}\right) .
\end{aligned}
$$

(vi) Note that $w(s) \simeq s^{q-1}$ and $\psi(s) \simeq \Phi(s)^{1-q}$ for $s \in\left(0, R_{1} / 2\right)$ in this case. We get from (7.6) that

$$
\begin{aligned}
A_{p, q}(t, x, y) & \simeq \frac{\psi(r)}{\Phi(r)} \int_{2 \phi^{-1}(1 / t)^{-1}}^{3 \Phi\left(\delta^{t}(y)\right) \wedge 4 \Phi(r)} s^{q-1} d s+\frac{\psi(r)}{\Phi(r)} \Phi\left(\delta^{t}(y)\right)^{q} \int_{3 \Phi\left(\delta^{t}(y)\right) \wedge 4 \Phi(r)}^{4 \Phi(r)} s^{-1} d s \\
& \simeq \frac{\Phi\left(\delta^{t}(y)\right)^{q}}{\Phi(r)^{q}} \log \left(e+\frac{\Phi(r)}{\Phi\left(\delta^{t}(y)\right)}\right) .
\end{aligned}
$$

(vii) Note that $w(s) \simeq s^{p-1}$ and $\psi(s) \simeq \Phi(s)^{1-p}$ for $s \in\left(0, R_{1}\right)$ in this case. By (17.6)), we obtain

$$
\begin{aligned}
A_{p, q}(t, x, y) \simeq & \frac{\psi(r)}{\Phi(r)} \int_{2 \phi^{-1}(1 / t)^{-1}}^{3 \Phi\left(\delta_{\wedge}^{t}(x, y)\right) \wedge 4 \Phi(r)} s^{p-1} d s+\frac{\psi(r)}{\Phi(r)} \Phi\left(\delta_{\wedge}^{t}(x, y)\right)^{p} \int_{3 \Phi\left(\delta_{\wedge}^{t}(x, y)\right) \wedge 4 \Phi(r)}^{3 \Phi\left(\delta_{\vee}^{t}(x, y)\right) \wedge 4 \Phi(r)} s^{-1} d s \\
& +\frac{\psi(r)}{\Phi(r)} \Phi\left(\delta_{\wedge}^{t}(x, y)\right)^{p} \Phi\left(\delta_{\vee}^{t}(x, y)\right)^{p} \int_{3 \Phi\left(\delta_{\vee}^{t}(x, y)\right) \wedge 4 \Phi(r)}^{4 \Phi(r)} s^{-1-p} d s \\
\simeq & \frac{\Phi\left(\delta_{\wedge}^{t}(x, y)\right)^{p}}{\Phi(r)^{p}} \log \left(e+\frac{\Phi\left(\delta_{\vee}^{t}(x, y)\right)}{\Phi\left(\delta_{\wedge}^{t}(x, y)\right)}\right) .
\end{aligned}
$$

(viii) Note that $w(s) \simeq s^{q-1}$ and $\psi(s) \simeq \Phi(s)^{1-q}$ for $s \in\left(0, R_{1}\right)$ in this case. If $\delta_{\vee}^{t}(x, y)=\delta^{t}(y)$, then we get from Lemma [5.1(i) and (7.6) that

$$
\begin{aligned}
A_{p, q}(t, x, y) \simeq & \frac{\psi(r)}{\Phi(r)} \int_{2 \phi^{-1}(1 / t)^{-1}}^{3 \Phi\left(\delta^{t}(y)\right) \wedge 4 \Phi(r)}\left(s \wedge \Phi\left(\delta^{t}(x)\right)\right)^{p} s^{q-p-1} d s \\
& +\frac{\psi(r)}{\Phi(r)} \Phi\left(\delta^{t}(x)\right)^{p} \Phi\left(\delta^{t}(y)\right)^{q} \int_{3 \Phi\left(\delta^{t}(y)\right) \wedge 4 \Phi(r)}^{4 \Phi(r)} s^{-p-1} d s \\
\simeq & \frac{\Phi\left(\delta^{t}(x)\right)^{p} \Phi\left(\delta^{t}(y)\right)^{q-p}}{\Phi(r)^{q}}=\frac{\Phi\left(\delta^{t}(y)\right)^{q-p} \Phi\left(\delta_{\wedge}^{t}(x, y)\right)^{p}}{\Phi(r)^{q}}
\end{aligned}
$$

If $\delta_{\wedge}^{t}(x, y)=\delta^{t}(y)$, then we get from (7.6) that

$$
\begin{aligned}
A_{p, q}(t, x, y) \simeq & \frac{\psi(r)}{\Phi(r)} \int_{2 \phi^{-1}(1 / t)^{-1}}^{3 \Phi\left(\delta^{t}(y)\right) \wedge 4 \Phi(r)} s^{q-1} d s+\frac{\psi(r)}{\Phi(r)} \Phi\left(\delta^{t}(y)\right)^{q} \int_{3 \Phi\left(\delta^{t}(y)\right) \wedge 4 \Phi(r)}^{3 \Phi\left(\delta^{t}(x)\right) \wedge 4 \Phi(r)} s^{-1} d s \\
& +\frac{\psi(r)}{\Phi(r)} \Phi\left(\delta^{t}(x)\right)^{p} \Phi\left(\delta^{t}(y)\right)^{q} \int_{3 \Phi\left(\delta^{t}(x)\right) \wedge 4 \Phi(r)}^{4 \Phi(r)} s^{-1-p} d s \\
\simeq & \frac{\Phi\left(\delta^{t}(y)\right)^{q}}{\Phi(r)^{q}} \log \left(e+\frac{\Phi\left(\delta^{t}(x)\right)}{\Phi\left(\delta^{t}(y)\right)}\right)=\frac{\Phi\left(\delta^{t}(y)\right)^{q-p} \Phi\left(\delta_{\wedge}^{t}(x, y)\right)^{p}}{\Phi(r)^{q}} \log \left(e+\frac{\Phi\left(\delta^{t}(x)\right)}{\Phi\left(\delta^{t}(y)\right)}\right) .
\end{aligned}
$$

(ix) Note that $w(s) \simeq s^{p-1}$ and $\psi(s) \simeq \Phi(s)^{1-p}$ for $s \in\left(0, R_{1}\right)$ in this case. If $\delta_{V}^{t}(x, y)=\delta^{t}(y)$, then by (7.6), it holds that

$$
A_{p, q}(t, x, y) \simeq \frac{\psi(r)}{\Phi(r)} \int_{2 \phi^{-1}(1 / t)^{-1}}^{3 \Phi\left(\delta^{t}(x)\right) \wedge 4 \Phi(r)} s^{p-1} d s+\frac{\psi(r)}{\Phi(r)} \Phi\left(\delta^{t}(x)\right)^{p} \int_{3 \Phi\left(\delta^{t}(x)\right) \wedge 4 \Phi(r)}^{3 \Phi\left(\delta^{t}(y)\right) \wedge 4 \Phi(r)} s^{-1} d s
$$




$$
\begin{aligned}
& +\frac{\psi(r)}{\Phi(r)} \Phi\left(\delta^{t}(x)\right)^{p} \Phi\left(\delta^{t}(y)\right)^{q} \int_{3 \Phi\left(\delta^{t}(y)\right) \wedge 4 \Phi(r)}^{4 \Phi(r)} s^{-1-q} d s \\
\simeq & \frac{\Phi\left(\delta^{t}(x)\right)^{p}}{\Phi(r)^{p}} \log \left(e+\frac{\Phi\left(\delta^{t}(y)\right)}{\Phi\left(\delta^{t}(x)\right)}\right)=\frac{\Phi\left(\delta_{\wedge}^{t}(x, y)\right)^{p}}{\Phi(r)^{p}} \log \left(e+\frac{\Phi\left(\delta^{t}(y)\right)}{\Phi\left(\delta^{t}(x)\right)}\right) .
\end{aligned}
$$

If $\delta_{\wedge}^{t}(x, y)=\delta^{t}(y)$, then we get from Lemma 5.1(ii) and (17.6) that

$$
\begin{aligned}
A_{p, q}(t, x, y) & \simeq \frac{\psi(r)}{\Phi(r)} \int_{2 \phi^{-1}(1 / t)^{-1}}^{3 \Phi\left(\delta^{t}(y)\right) \wedge 4 \Phi(r)} s^{p-1} d s+\frac{\psi(r)}{\Phi(r)} \Phi\left(\delta^{t}(y)\right)^{q} \int_{3 \Phi\left(\delta^{t}(y)\right) \wedge 4 \Phi(r)}^{4 \Phi(r)}\left(1 \wedge \frac{\Phi\left(\delta^{t}(x)\right)}{s}\right)^{p} s^{p-q-1} d s \\
& \simeq \frac{\Phi\left(\delta^{t}(y)\right)^{p}}{\Phi(r)^{p}}=\frac{\Phi\left(\delta_{\wedge}^{t}(x, y)\right)^{p}}{\Phi(r)^{p}} .
\end{aligned}
$$

Example 7.2. Let $d, \alpha>0, \beta \in(0,1)$ and $p, q \geq 0$ such that $p+q>0$. Suppose that for every $r_{0} \geq 1$, there are comparability constants such that

$$
V(x, r) \simeq r^{d}, \quad x \in D, 0<r<r_{0} .
$$

Let $Y^{D}$ be a Hunt process in $D$ and $S=\left(S_{t}\right)_{t \geq 0}$ be an independent driftless subordinator with Laplace exponent $\phi$. Suppose that the tail $w$ of the Lévy measure of $S$ satisfies

$$
w(r) \simeq r^{-\beta}, \quad 0<r<r_{1},
$$

for some $r_{1}>0$. Suppose that the heat kernel $p_{D}(t, x, y)$ of $Y^{D}$ satisfies either $\mathbf{H K}_{\mathbf{B}}^{\mathbf{h}_{\mathbf{p}, \mathbf{q}}}$ or $\mathbf{H K}_{\mathbf{U}}^{\mathbf{h}_{\mathbf{p}, \mathbf{q}}}$ with $\Phi(r)=\Psi(r)=r^{\alpha}$ where the boundary function $h_{p, q}$ is defined as (3.8). When $\mathbf{H K}_{\mathbf{U}}^{\mathbf{h}_{\mathbf{p}, \mathbf{q}}}$ is satisfied, we also assume that (7.8) and (7.9) hold for all $r>0$. See Example 3.9 for concrete examples of $Y^{D}$. By switching the roles of $x$ and $y$ if needed, without loss of generality, we assume that $q \geq p$.

Let $q(t, x, y), J(x, y)$ and $G_{D}(x, y)$ be the heat kernel, the jump kernel and the Green function of the subordinate process $X_{t}:=Y_{S_{t}}^{D}$ respectively. Using our theorems in Sections 4 and 5 , and Lemma 7.1 , we get explicit estimates on $q(t, x, y), J(x, y)$ and $G_{D}(x, y)$. We list them in terms of the range of $p+q$, similar to the format of the Green function estimates for Dirichlet forms degenerate at the boundary in [44.

In particular, by putting $p=q=1 / 2$, we get Theorem 1.1 .

We first give the Green function estimates. Define

$$
\mathfrak{g}(x, y):=\left(1 \wedge \frac{\delta(x)}{\rho(x, y)}\right)^{\alpha p}\left(1 \wedge \frac{\delta(y)}{\rho(x, y)}\right)^{\alpha q} \times \begin{cases}\rho(x, y)^{\alpha \beta-d}, & d>\alpha \beta, \\ \log \left(e+\frac{\delta_{\vee}(x, y)}{\rho(x, y)}\right), & d=\alpha \beta, \\ {\left[\rho(x, y) \vee \delta_{\vee}(x, y)\right]^{\alpha \beta-d},} & d<\alpha \beta .\end{cases}
$$

When $C_{0}=0$, by Theorem 5.8 and Example 5.5, for all $x, y \in D$, if $d>\alpha(\beta-p-q)$, then

$$
G_{D}(x, y) \simeq \mathfrak{g}(x, y)
$$

and if $d \leq \alpha(\beta-p-q)$, then

$$
G_{D}(x, y) \simeq \begin{cases}\delta(x)^{\alpha p} \delta(y)^{\alpha q} \log \left(e+\frac{\operatorname{diam}(D)}{\rho(x, y) \vee \delta_{\vee}(x, y)}\right), & d=\alpha(\beta-p-q) \text { and } \mathbf{H K}_{\mathbf{B}}^{\mathbf{h}_{\mathbf{p}, \mathbf{q}}} \text { holds } \\ \delta(x)^{\alpha p} \delta(y)^{\alpha q}, & d<\alpha(\beta-p-q) \text { and } \mathbf{H K}_{\mathbf{B}}^{\mathbf{h}_{\mathbf{p}, \mathbf{q}}} \text { holds } \\ \infty, & d \leq \alpha(\beta-p-q) \text { and } \mathbf{H K}_{\mathbf{U}}^{\mathbf{h}_{\mathbf{p}, \mathbf{q}}} \text { holds }\end{cases}
$$

Now assume that $C_{0}=1$. If $p+q<\beta+1$, then using Theorem 5.8 and Example 5.5 again, we see that (7.10) and (7.11) also hold. If $p+q=\beta+1$ and $q<\beta+1$ (so that (H2**) holds, cf. Example [5.9), then by Theorem [5.11, for all $x, y \in D$,

$$
G_{D}(x, y) \simeq \mathfrak{g}(x, y) \log \left(e+\frac{\rho(x, y)}{\delta_{\vee}(x, y)}\right) .
$$

If $p+q>\beta+1$ and $q<\beta+1$ (so, again, (H2**) holds), then by Theorem 5.10 and (5.18), for all $x, y \in D$,

$$
G_{D}(x, y) \simeq\left(1 \wedge \frac{\delta \vee(x, y)}{\rho(x, y)}\right)^{-\alpha(p+q-\beta-1)} \mathfrak{g}(x, y) .
$$


The unusual form of the estimates in (7.12)-(7.13) should be compared with similar estimates of the Green function obtained in a different context in [44, Theorem $1.1(2),(3)]$. Such estimates lead to anomalous boundary behavior of the corresponding Green potential, cf. 1].

Next, we get heat kernel estimates from Lemma 7.1. Corollary 4.4 and Theorem 4.7. By the definition (7.1), since $\psi(r) \simeq r^{\alpha \beta}$ for $r \in(0,1]$, we have for all $(t, x, y) \in(0,1] \times D \times D$,

$$
\delta^{t}(x) \simeq \delta_{D}(x) \vee t^{1 /(\alpha \beta)}, \quad \delta_{\vee}^{t}(x, y) \simeq \delta_{\vee}(x, y) \vee t^{1 /(\alpha \beta)}, \quad \delta_{\wedge}^{t}(x, y) \simeq \delta_{\wedge}(x, y) \vee t^{1 /(\alpha \beta)} .
$$

Moreover, when $\mathbf{H K}_{\mathbf{U}}^{\mathbf{h}_{\mathbf{p}}}$ holds, since $\psi(r) \simeq r^{\alpha \beta}$ for all $r>0$ in this case, (17.14) holds for all $(t, x, y) \in$ $(0, \infty) \times D \times D$.

(Small time estimates) The following estimates hold for all $(t, x, y) \in(0,1] \times D \times D$ :

$$
\begin{aligned}
q(t, x, y) & \simeq\left(1 \wedge \frac{\delta(x)}{t^{1 /(\alpha \beta)}}\right)^{\alpha p}\left(1 \wedge \frac{\delta(y)}{t^{1 /(\alpha \beta)}}\right)^{\alpha q} B_{p, q}\left(t, x, y ; C_{0}\right)\left(t^{-d /(\alpha \beta)} \wedge \frac{t}{\rho(x, y)^{d+\alpha \beta}}\right) \\
& \simeq\left(1 \wedge \frac{\delta(x)}{t^{1 /(\alpha \beta)}}\right)^{\alpha p}\left(1 \wedge \frac{\delta(y)}{t^{1 /(\alpha \beta)}}\right)^{\alpha q}\left(t^{-d /(\alpha \beta)} \wedge \frac{t B_{p, q}\left(t, x, y ; C_{0}\right)}{\rho(x, y)^{d+\alpha \beta}}\right),
\end{aligned}
$$

where

$$
B_{p, q}(t, x, y ; 0):=\left(1 \wedge \frac{\delta_{\wedge}^{t}(x, y)}{\rho(x, y)}\right)^{\alpha p}\left(1 \wedge \frac{\delta_{\vee}^{t}(x, y)}{\rho(x, y)}\right)^{\alpha p}\left(1 \wedge \frac{\delta^{t}(y)}{\rho(x, y)}\right)^{\alpha(q-p)}
$$

and

$$
\begin{aligned}
& B_{p, q}(t, x, y ; 1):= \\
& \begin{cases}\left(1 \wedge \frac{\delta_{\wedge}^{t}(x, y)}{\rho(x, y)}\right)^{\alpha(1-\beta)}, & q \geq p>1-\beta, \\
\left(1 \wedge \frac{\delta_{\wedge}^{t}(x, y)}{\rho(x, y)}\right)^{\alpha(1-\beta)} \log \left(e+\frac{\rho(x, y) \wedge \delta^{t}(y)}{\delta^{t}(x)}\right), & q>1-\beta=p, \\
\left(1 \wedge \frac{\delta_{\wedge}^{t}(x, y)}{\rho(x, y)}\right)^{\alpha p}\left(1 \wedge \frac{\delta^{t}(y)}{\rho(x, y)}\right)^{\alpha(1-\beta-p)}, & q>1-\beta>p, \\
\left(1 \wedge \frac{\delta_{\wedge}^{t}(x, y)}{\rho(x, y)}\right)^{\alpha(1-\beta)} \log \left(e+\frac{\rho(x, y) \wedge \delta_{\vee}^{t}(x, y)}{\delta_{\wedge}^{t}(x, y)}\right), & q=1-\beta=p, \\
\left(1 \wedge \frac{\delta_{\wedge}^{t}(x, y)}{\rho(x, y)}\right)^{\alpha p}\left(1 \wedge \frac{\delta^{t}(y)}{\rho(x, y)}\right)^{\alpha(1-\beta-p)} \log \left(e+\frac{\delta^{t}(x) \wedge \rho(x, y)}{\delta^{t}(y)}\right), & 1-\beta=q>p>0, \\
\left(1 \wedge \frac{\delta_{\wedge}^{t}(x, y)}{\rho(x, y)}\right)^{\alpha p}\left(1 \wedge \frac{\delta_{\vee}^{t}(x, y)}{\rho(x, y)}\right)^{\alpha(1-\beta-q)}\left(1 \wedge \frac{\delta^{t}(y)}{\rho(x, y)}\right)^{\alpha(q-p)}, & 1-\beta>q>1-\beta-p, \\
\left(1 \wedge \frac{\delta_{\wedge}^{t}(x, y)}{\rho(x, y)}\right)^{\alpha p}\left(1 \wedge \frac{\delta_{\vee}^{t}(x, y)}{\rho(x, y)}\right)^{\alpha p}\left(1 \wedge \frac{\delta^{t}(y)}{\rho(x, y)}\right)^{\alpha(q-p)} \log \left(e+\frac{\rho(x, y)}{\delta_{\vee}^{t}(x, y)}\right), & 1-\beta>q=1-\beta-p, \\
\left(1 \wedge \frac{\delta^{t}(y)}{\rho(x, y)}\right)^{\alpha q} \log \left(e+\frac{\rho(x, y)}{\delta^{t}(y)}\right), & q=1-\beta=1-\beta-p, \\
\left(1 \wedge \frac{\delta_{\wedge}^{t}(x, y)}{\rho(x, y)}\right)^{\alpha p}\left(1 \wedge \frac{\delta_{\vee}^{t}(x, y)}{\rho(x, y)}\right)^{\alpha p}\left(1 \wedge \frac{\delta^{t}(y)}{\rho(x, y)}\right)^{\alpha(q-p)}, & q<1-\beta-p .\end{cases}
\end{aligned}
$$

(Large time estimates) If $\mathbf{H K}_{\mathbf{B}}^{\mathbf{h}_{\mathbf{p}}}$ holds, then for all $(t, x, y) \in[1, \infty) \times D \times D$,

$$
q(t, x, y) \simeq e^{-t \phi\left(\lambda_{D}\right)} \delta(x)^{\alpha p} \delta(y)^{\alpha q},
$$

and if $\mathbf{H K}_{\mathbf{U}}^{\mathbf{h}_{\mathbf{p}}}$ holds, then (7.15) holds for all $(t, x, y) \in(0, \infty) \times D \times D$.

Lastly, we give estimates on the jump kernel $J(x, y)$. By Theorem 4.1 and the fact that $\mathcal{B}_{h}^{*}(x, y) \simeq$ $\mathcal{B}_{h}(0, x, y)$ for $x, y \in D$, we deduce from (7.15) that for any $q \geq p \geq 0$,

$$
J(x, y) \simeq \frac{B_{p, q}\left(0, x, y ; C_{0}\right)}{\rho(x, y)^{d+\alpha \beta}}, \quad x, y \in D .
$$


Example 7.3. Let $D:=\left\{x \in \mathbb{R}^{d}: x_{d}>0\right\}$ be the upper half space in $\mathbb{R}^{d}$ and $q \in[\alpha-1, \alpha) \cap(0, \alpha)$. We recall the process $Y^{D}$ from Example 3.9 (b-4), which corresponds to the Feynman-Kac semigroup of the part process $Z^{D}$, in $D$, of the reflected isotropic $\alpha$-stable process in $\bar{D}$ via the multiplicative functional $\exp \left(-\int_{0}^{t} C(d, \alpha, q)\left(Z_{s}^{D}\right)_{d}^{-\alpha} d s\right)$, where the positive constants $C(d, \alpha, q)$ is defined on [25, p. 233]. It is easy to see that $Y^{D}$ satisfies the scaling property and horizontal translation invariance, more precisely, for any $\lambda>0$, the transition density $p_{D}$ of $Y^{D}$ satisfies

$$
p_{D}\left(\lambda^{\alpha}, \lambda x, \lambda y\right)=p_{D}(t, x, y) \lambda^{-d}, \quad t>0, x, y \in D
$$

and

$$
p_{D}(t, x+(\widetilde{z}, 0), y+(\widetilde{z}, 0))=p_{D}(t, x, y), \quad t>0, x, y \in D, \widetilde{z} \in \mathbb{R}^{d-1} .
$$

Let $S=\left(S_{t}\right)_{t \geq 0}$ be a $\beta$-stable subordinator independent of the process $Y^{D}, \beta \in(0,1)$. Then the process $X_{t}:=Y_{S_{t}}^{D}$ falls into the framework of the present paper and thus we can get sharp two-sided estimates on the jump kernel, heat kernel and Green functions of $X$. By using the scaling property and horizontal translation invariance of $p_{D}$ above, we can show that the killing function $\kappa(x)$ of $X$ is given by

$$
\kappa(x)=C x_{d}^{-\alpha \beta}, \quad x \in D
$$

for some constant $C \in(0, \infty)$. One can check, by following arguments at the end of [43, Section 2], that the jump kernel of the subordinate process $X_{t}:=Y_{S_{t}}^{D}$ satisfies assumptions in [44, (A1)-(A4)].

Moreover, by comparing the Green function estimates in [44, Theorem 1.1] with the Green function function estimates in Example 7.2, one can see that the value of the constant in the critical killing potential is indeed related to the power of the decay correctly. Thus, instead of computing the constant $C$ of the killing function $\kappa(x)$, we see that the exponent $p$ in [44] should be $q$ (and the constant $\alpha$ in [44] is equal to $\alpha \beta$ in the present case) and we can use [44, Theorems 1.2 and 1.3] directly. Therefore, by checking the range of $p=q / \alpha$ in the jump kernel estimates in Example 7.2 and [44, Theorems 1.2 and 1.3], from [44, Theorems 1.2 and 1.3] we obtain the following corollary. See [44, Theorem 1.2] for the precise statement of the scale-invariant boundary Harnack principle.

Corollary 7.4. Suppose $d>\alpha$ and $X$ is the process defined above. Let $\beta_{1 / 2}:=\beta \vee 1 / 2$. If $q \in$ $[\alpha-1, \alpha) \cap\left((\alpha \beta-1)_{+}, \alpha \beta_{1 / 2}\right)$, then the scale-invariant boundary Harnack principle is valid for $X$. If $q \in\left[\alpha \beta_{1 / 2} \vee(\alpha-1), \alpha\right)$, then the non-scale-invariant boundary Harnack principle is not valid for $X$.

Note that constants $\left(\beta_{1}, \beta_{2}\right)$ in [44] are $(\alpha-\alpha \beta, 0)$ for $\alpha(1-\beta) \leq q<\alpha,(q, \alpha-q-\alpha \beta)$ for $\alpha(1-\beta) / 2<$ $q<\alpha(1-\beta)$ and $(q, q)$ for $0 \leq q \leq \alpha(1-\beta) / 2$.

The next example illustrates that any of the four terms in (4.12) can not dominates all the other terms in general.

Example 7.5. Let $0<\beta<1<L$ and $S$ be a subordinator with tail Lévy measure $w$ satisfying

$$
w(r) \simeq r^{-\beta} \wedge r^{-L}, \quad r>0 .
$$

Let $0<\alpha \leq K$. Suppose that the heat kernel $p_{D}(t, x, y)$ of $Y^{D}$ enjoys the estimate $\mathbf{H K}_{\mathbf{U}}^{\mathbf{h}_{1 / 2}}$ with

$$
\Phi(r)=r^{\alpha}, \quad \Psi(r)=r^{\alpha} \vee r^{K}, \quad r>0 .
$$

Examples of such $Y^{D}$ can be found in [38, Theorem 1.4] where Dirichlet heat kernel estimates for a large class of subordinate Brownian motions are treated. Recall that $\psi(r):=\phi(1 / \Phi(r))^{-1}$. Note that $($ Poly- $\infty$ ) holds, and by (2.1),

$$
\begin{array}{rlrl}
\phi(1 / r) & \simeq\left\{\begin{array}{lll}
r^{-\beta}, & \text { if } r \leq 1 \\
r^{-1}, & \text { if } r>1,
\end{array}\right. \\
\phi^{-1}(1 / r)^{-1} \simeq\left\{\begin{array}{lll}
r^{1 / \beta}, & \text { if } r \leq 1 \\
r, & \text { if } r>1,
\end{array}\right. & \psi(r) \simeq \begin{cases}r^{\alpha \beta}, & \text { if } r \leq 1 \\
r^{\alpha}, & \text { if } r>1,\end{cases} \\
\text { if } r(r) \simeq \begin{cases}r^{1 /(\alpha \beta)}, & \text { if } r \leq 1 \\
r^{1 / \alpha}, & \text { if } r>1 .\end{cases}
\end{array}
$$

Let $q(t, x, y)$ be the heat kernel of the subordinate process $X_{t}:=Y_{S_{t}}^{D}$. We get global estimates on $q(t, x, y)$ from Theorem 4.3 and Lemma 7.1. Write the terms on the right-hand side of (4.12) respectively as

$$
A_{1}=\frac{C_{0} t \mathcal{B}_{h}(t, x, y)}{V(x, \rho(x, y)) \Psi(\rho(x, y))}, \quad \quad A_{2}=\frac{C_{0} \phi^{-1}(1 / t)^{-1} h\left(\phi^{-1}(1 / t)^{-1}, x, y\right)}{V(x, \rho(x, y)) \Psi(\rho(x, y))}
$$




$$
A_{3}=\frac{h\left(\phi^{-1}(1 / t)^{-1}, x, y\right)}{V\left(x, \psi^{-1}(t)\right)} \exp \left(-c \frac{\rho(x, y)^{2}}{\psi^{-1}(t)^{2}}\right), \quad A_{4}=\frac{\operatorname{th}(\Phi(\rho(x, y)), x, y) w(\Phi(\rho(x, y)))}{V(x, \rho(x, y))} .
$$

(Small time estimates) Note that for any $R_{1}>0, w$ satisfies (Poly- $R_{1}$ ) with both the upper and the lower index equal to $\beta \in(0,1)$. Using this fact, it is easy to see that for all $(t, x, y) \in(0,1 / \phi(4)] \times D \times D$ with $\rho(x, y) \geq t^{1 /(\alpha \beta)}, A_{4}$ dominates $A_{3}$ and $A_{1}$ dominates $A_{2}$. Thus, the following estimates hold for all $(t, x, y) \in(0,1 / \phi(4)] \times D \times D$.

(i) If $\rho(x, y) \leq \phi(4)^{-1 /(\alpha \beta)}$, then

$q(t, x, y) \simeq\left(1 \wedge \frac{\delta(x)}{t^{1 /(\alpha \beta)}}\right)^{\alpha / 2}\left(1 \wedge \frac{\delta(y)}{t^{1 /(\alpha \beta)}}\right)^{\alpha / 2} B_{1 / 2,1 / 2}\left(t, x, y ; C_{0}\right)\left(\frac{1}{V\left(x, t^{1 /(\alpha \beta)}\right)} \wedge \frac{t}{V(x, \rho(x, y)) \rho(x, y)^{\alpha \beta}}\right)$, where the function $B_{1 / 2,1 / 2}\left(t, x, y ; C_{0}\right)$ is defined as in (7.16) and (7.17).

(ii) Let $\rho(x, y)>\phi(4)^{-1 /(\alpha \beta)}$. Since $L>1$, we see that for all $u>1$,

$$
\int_{u}^{\infty} h_{1 / 2}(s, x, y) s^{-L} d s \leq h_{1 / 2}(u, x, y) \int_{u}^{\infty} s^{-L} d s \leq \frac{u^{1-L}}{L-1} h_{1 / 2}(u, x, y) .
$$

Thus, we get from (5.2), (7.5) and (7.18) that

$$
\begin{aligned}
\mathcal{B}_{h_{1 / 2,1 / 2}}(t, x, y) & \simeq \int_{2 \phi^{-1}(1 / t)^{-1}}^{1} h_{1 / 2}(s, x, y) s^{-\beta} d s+\int_{1}^{4 \rho(x, y)^{\alpha}} h_{1 / 2}(s, x, y) s^{-L} d s \\
& \simeq \int_{2 \phi^{-1}(1 / t)^{-1}}^{1} h_{1 / 2}(s, x, y) s^{-\beta} d s+h_{1 / 2}(1, x, y) \\
& \simeq \int_{2 \phi^{-1}(1 / t)^{-1}}^{1} h_{1 / 2}(s, x, y) s^{-\beta} d s \\
& =h_{1 / 2}\left(2 \phi^{-1}(1 / t)^{-1}, x, y\right) \int_{2 \phi^{-1}(1 / t)^{-1}}^{1}\left(1 \wedge \frac{\delta_{\wedge}^{t}(x, y)^{\alpha}}{s}\right)^{1 / 2}\left(1 \wedge \frac{\delta_{\vee}^{t}(x, y)^{\alpha}}{s}\right)^{1 / 2} s^{-\beta} d s .
\end{aligned}
$$

In the end, by similar arguments to that given in the proof of Lemma 7.1, using (7.18), we conclude that

$$
\begin{aligned}
q(t, x, y) & \simeq\left(1 \wedge \frac{\delta(x)}{t^{1 /(\alpha \beta)}}\right)^{\frac{\alpha}{2}}\left(1 \wedge \frac{\delta(y)}{t^{1 /(\alpha \beta)}}\right)^{\frac{\alpha}{2}} \\
\times[(1 & \left.\left.\wedge \frac{\delta_{\wedge}(x, y) \vee t^{1 /(\alpha \beta)}}{\rho(x, y)}\right)^{\frac{\alpha}{2}}\left(1 \wedge \frac{\delta \vee(x, y) \vee t^{1 /(\alpha \beta)}}{\rho(x, y)}\right)^{\frac{\alpha}{2}} \frac{t}{V(x, \rho(x, y)) \rho(x, y)^{\alpha L}}+\frac{C_{0} t F_{\beta}(t, x, y)}{V(x, \rho(x, y)) \rho(x, y)^{K}}\right],
\end{aligned}
$$

where

$$
F_{\beta}(t, x, y):= \begin{cases}\left(1 \wedge\left(\delta_{\wedge}(x, y) \vee t^{1 /(\alpha \beta)}\right)\right)^{\alpha / 2}\left(1 \wedge\left(\delta_{\vee}(x, y) \vee t^{1 /(\alpha \beta)}\right)\right)^{\alpha / 2-\alpha \beta}, & \text { if } \beta<1 / 2, \\ \left(1 \wedge\left(\delta_{\wedge}(x, y) \vee t^{1 /(\alpha \beta)}\right)\right)^{\alpha / 2} \log \left(e+\frac{1 \wedge\left(\delta_{\vee}(x, y) \vee t^{1 /(\alpha \beta)}\right)}{1 \wedge\left(\delta_{\wedge}(x, y) \vee t^{1 /(\alpha \beta)}\right)}\right), & \text { if } \beta=1 / 2, \\ \left(1 \wedge\left(\delta_{\wedge}(x, y) \vee t^{1 /(\alpha \beta)}\right)\right)^{\alpha-\alpha \beta}, & \text { if } \beta>1 / 2 .\end{cases}
$$

Therefore, when $C_{0}=1$, since $K$ or $L$ may be arbitrarily large number, one should keep both terms $A_{1}$ and $A_{4}$ for off-diagonal estimates in general.

(Large time estimates) By (7.19), we see that for all $(t, x, y) \in[1 / \phi(4), \infty) \times D \times D$ with $\rho(x, y) \geq t^{1 / \alpha}$, $A_{2}$ dominates $A_{1}$ since $t \phi^{-1}(1 / t)^{-L} \simeq t^{1-L} \leq \phi(4)^{L-1}$ in this case. Thus, the following estimates hold for all $(t, x, y) \in[1 / \phi(4), \infty) \times D \times D$.

(i) If $\rho(x, y)<t^{1 / \alpha}$, then

$$
q(t, x, y) \simeq\left(1 \wedge \frac{\delta(x)}{t^{1 / \alpha}}\right)^{\alpha / 2}\left(1 \wedge \frac{\delta(y)}{t^{1 / \alpha}}\right)^{\alpha / 2} \frac{1}{V\left(x, t^{1 / \alpha}\right)} .
$$

(ii) If $\rho(x, y) \geq t^{1 / \alpha}$, then

$$
\begin{aligned}
q(t, x, y) \asymp( & \left.1 \wedge \frac{\delta(x)}{t^{1 / \alpha}}\right)^{\alpha / 2}\left(1 \wedge \frac{\delta(y)}{t^{1 / \alpha}}\right)^{\alpha / 2}\left[\frac{C_{0} t}{V(x, \rho(x, y)) \rho(x, y)^{K}}+\frac{1}{V\left(x, t^{1 / \alpha}\right)} \exp \left(-c \frac{\rho(x, y)^{2}}{t^{2 / \alpha}}\right)\right. \\
& \left.+\left(1 \wedge \frac{\delta(x) \vee t^{1 / \alpha}}{\rho(x, y)}\right)^{\alpha / 2}\left(1 \wedge \frac{\delta(y) \vee t^{1 / \alpha}}{\rho(x, y)}\right)^{\alpha / 2} \frac{t}{V(x, \rho(x, y)) \rho(x, y)^{\alpha L}}\right] .
\end{aligned}
$$


In particular, one should keep all terms $A_{2}, A_{3}$ and $A_{4}$ for off-diagonal estimates in general.

Note that, in case of $C_{0}=1$ and $L \geq K / \alpha$, we see from (7.20) and (7.21) that

$$
q(t, x, y) \asymp p_{D}(c t, x, y), \quad t \geq 1 / \phi(4), x, y \in D .
$$

Acknowledgment: We thank the referee for the comments on the first version of this paper. The third-named author thanks Professor Habib Maagli for pointing a mistake in Theorem 3.10 of [49], which led to a mistake in Theorem 4.6 there. Part of the research for this paper was done while the thirdnamed author was visiting Jiangsu Normal University, where he was partially supported by a grant from the National Natural Science Foundation of China (11931004) and by the Priority Academic Program Development of Jiangsu Higher Education Institutions.

\section{REFERENCES}

[1] N. Abatangelo, D. Gomez-Castro and J. L Vázquez. Singular boundary behaviour and large solutions for fractional elliptic equations. arXiv: 1910.00366

[2] K. Bogdan, T. Grzywny, and M. Ryznar. Heat kernel estimates for the fractional Laplacian with Dirichlet conditions. Ann. Probab. 38 (2010), 1901-1923.

[3] K. Bogdan, T. Grzywny, and M. Ryznar. Dirichlet heat kernel for unimodal Lévy processes. Stochastic Process. Appl. 124 (2014), 3612-3650.

[4] K. Bogdan, T. Grzywny, and M. Ryznar. Barriers, exit time and survival probability for unimodal Lévy processes. Probab. Theory Related Fields 162 (2015), 155-198.

[5] M. Bonforte, Y. Sire and J. L. Vázquez. Existence, uniqueness and asymptotic behaviour for fractional porous medium equations on bounded domains. Discrete Contin. Dyn. Syst. 35 (2015), 5725-5767.

[6] N. Bouleau. Quelques résultats probabilistes sur la subordination au sens de Bochner. Seminar on potential theory, Paris, No. 7, 54-81, Lecture Notes in Math., 1061, Springer, Berlin, 1984.

[7] Z.-Q. Chen and P. Kim. Global Dirichlet heat kernel estimates for symmetric Lévy processes in half-space. Acta Appl. Math. 146 (2016), 113-143

[8] Z.-Q. Chen, P. Kim, T. Kumagai and J. Wang. Heat kernel estimates for time fractional equations. Forum Math. 30 (2018), 1163-1192.

[9] Z.-Q. Chen, P. Kim and R. Song. Heat kernel estimates for the Dirichlet fractional Laplacian. J. Eur. Math. Soc. 12 (2010), 1307-1329.

[10] Z.-Q. Chen, P. Kim and R. Song. Two-sided heat kernel estimates for censored stable-like processes. Probab. Theory Relat. Fields, 146 (2010), 361-399.

[11] Z.-Q. Chen, P. Kim and R. Song. Heat kernel estimates for $\Delta+\Delta^{\alpha / 2}$ in $C^{1,1}$ open sets, J. Lond. Math. Soc. (2) 84 (2011), no. 1, 58-80.

[12] Z.-Q. Chen, P. Kim and R. Song. Dirichlet heat kernel estimates for fractional Laplacian with gradient perturbation, Ann. Probab. 40 (2012), 2483-2538.

[13] Z.-Q. Chen, P. Kim and R. Song. Global heat kernel estimates for $\Delta+\Delta^{\alpha / 2}$ in half-space-like domains. Elect. J. Probab. 17 (2012), No. 32, 1-32.

[14] Z.-Q. Chen, P. Kim and R. Song. Dirichlet heat kernel estimates for rotationally symmetric Lévy processes. Proc. Lond. Math. Soc. 109 (2014), 90-120.

[15] Z.-Q. Chen, P. Kim and R. Song. Dirichlet Heat Kernel Estimates for Subordinate Brownian Motions with Gaussian Components. J. Reine Angew. Math. 711 (2016), 111-138.

[16] Z.-Q. Chen, P. Kim and T. Kumagai. On heat kernel estimates and parabolic Harnack inequality for jump processes on metric measure spaces. Acta Math. Sin. (Engl. Ser.) 25 (2009), 1067-1086.

[17] Z.-Q. Chen and T. Kumagai. Heat kernel estimates for stable-like processes on d-sets. Stochastic Process. Appl. 108 (2003), no. 1, 27-62.

[18] Z.-Q. Chen, T. Kumagai, and J. Wang. Stability of heat kernel estimates for symmetric non-local Dirichlet forms. Mem. Amer. Math. Soc. 271 (2021), no. 1330.

[19] Z.-Q. Chen, T. Kumagai, and J. Wang. Stability of parabolic Harnack inequalities for symmetric non-local Dirichlet forms. J. Eur. Math. Soc. 22 (2020), 3747-3803.

[20] Z.-Q. Chen and R. Song. Intrinsic ultracontractivity and conditional gauge for symmetric stable processes. J. Funct. Anal., 150 (1997), 204-239.

[21] Z.-Q. Chen and J. Tokle. Global heat kernel estimates for fractional Laplacians in unbounded open sets. Probab. Theory Relat. Fields, 149 (2011), 373-395.

[22] S. Cho and P. Kim. Estimates on the tail probabilities of subordinators and applications to general time fractional equations. Stochastic Process. Appl. 130 (2020), 4392-4443.

[23] S. Cho and P. Kim. Estimates on transition densities of subordinators with jumping density decaying in mixed polynomial orders. Stochastic Process. Appl. 139 (2021), 229-279.

[24] S. Cho, P. Kim. and H. Park. Two-sided estimates on Dirichlet heat kernels for time-dependent parabolic operators with singular drifts in $C^{1, \alpha}$-domains. J. Differential Equations, 252 (2012), 1101-1145.

[25] S. Cho, P. Kim, R. Song, R. and Z. Vondraček. Factorization and estimates of Dirichlet heat kernels for non-local operators with critical killings. J. Math. Pures Appl. 143 (2020), 208-256. 
[26] K. L. Chung and Z. X. Zhao. From Brownian motion to Schrödinger's equation. Springer-Verlag, Berlin, 1995.

[27] E. B. Davies. Heat kernels and spectral theory. Cambridge University Press, Cambridge, 1989.

[28] J. Davila and L. Dupaigne. Hardy-type inequalities. J. Eur. Math. Soc. 6 (2004), 335—365.

[29] S. Filippas, L. Moschini and A.Tertikas. Sharp two-sided heat kernel estimates for critical Schrödinger operators on bounded domains. Comm. Math. Phys. 273 (2007), 237-281.

[30] P. Gyrya and L. Saloff-Coste. Neumann and Dirichlet heat kernels in inner uniform domains. Astérisque, 336 (2011), viii+144 pp.

[31] T. Grzywny, K.-Y. Kim and P. Kim. Estimates of Dirichlet heat kernel for symmetric Markov processes. Stochastic Process. Appl. 130 (2020), no. 1, 431-470.

[32] N. C. Jain and W. E. Pruitt. Lower tail probability estimates for subordinators and nondecreasing random walks. Ann. Probab. 15 (1987), 75-101.

[33] T. Jakubowski and J. Wang. Heat kernel estimates of fractional Schrödinger operators with negative Hardy potential. Potential Anal. 53 (2020), 997-1024.

[34] K. Kaleta and P. Sztonyk. Estimates of transition densities and their derivatives for jump Lévy processes. J. Math. Anal. Appl. 431 (2015), 260-282.

[35] K.-Y. Kim. Global heat kernel estimates for symmetric Markov processes dominated by stable-like processes in exterior $C^{1, \eta}$ open sets. Potential Anal. 43 (2015), 127-148.

[36] K.-Y. Kim and P. Kim. Two-sided estimates for the transition densities of symmetric Markov processes dominated by stable-like processes in $C^{1, \eta}$ open sets. Stochastic Process. Appl. 124 (2014), 3055-3083. Erratum to "Two-sided estimates for the transition densities of symmetric Markov processes dominated by stable-like processes in $C^{1, \eta}$ open sets" [Stochastic Process. Appl. 124 (2014) 3055-3083]. Stochastic Process. Appl. 129 (2019), 2228-2230.

[37] P. Kim and A. Mimica. Green function estimates for subordinate Brownian motions: Stable and beyond. Trans. Amer. Math. Soc. 366 (2014), No. 8, 4383-4422.

[38] P. Kim and A. Mimica. Estimates of Dirichlet heat kernels for subordinate Brownian motions. Electron. J. Probab. 23 (2018), No. 64, 45 pp.

[39] P. Kim and R. Song. Dirichlet heat kernel estimates for stable processes with singular drift in unbounded $C^{1,1}$ open sets. Potential Anal. 41 (2014), 555-581.

[40] P. Kim, R. Song and Z. Vondraček. Global uniform boundary Hanack principle with explicit decay rate and its application. Stoch. Proc. Appl. 124 (2014), 235-267.

[41] P. Kim, R. Song and Z. Vondraček. Potential theory of subordinate killed Brownian motions. Trans. Amer. Math. Soc. 371 (2019), 3917-3969.

[42] P. Kim, R. Song and Z. Vondraček. On the boundary theory of subordinate killed Lévy processes. Potential Anal. 53 (2020), 131-181.

[43] P. Kim, R. Song and Z. Vondraček. On potential theory of Markov processes with jump kernels decaying at the boundary. To appear in Potential Anal. DOI: 10.1007/s11118-021-09947-8 (2021).

[44] P. Kim, R. Song and Z. Vondraček. Sharp two-sided Green function estimates for Dirichlet forms degenerate at the boundary. arXiv:2011.00234v3 (2021).

[45] D. Kinzebulatov and Yu. A. Semënov. Fractional Kolmogorov operator and desingularizing weights. arXiv:2005.11199v4 (2020).

[46] G. Metafune, L. Negro and C. Spina. Sharp kernel estimates for elliptic operators with second-order discontinuous coefficients. J. Evol. Equ. 18 (2018), 467-514.

[47] A. Mimica. Heat kernel estimates for subordinate Brownian motions. Proc. Lond. Math. Soc. (3) 113 (2016), $627-648$.

[48] A. Miyake. The subordination of Lévy system for Markov processes. Proc. Japan Acad. 45 (1969), 601-604.

[49] R. Song. Sharp bounds on the density, Green function and jumping function of subordinate killed BM. Probab. Theory Related Fields, Vol. 128 (2004), No. 4, 606-628.

[50] R. Song and Z. Vondraček. On the relationship between subordinate killed and killed subordinate processes. Electron. Commun. Probab. 13 (2008), 325-336.

[51] N. Th. Varopoulos. Gaussian estimates in Lipschitz domains. Can. J. Math. 55 (2003) 401-431.

[52] Z. Zhao. Green function for Schrödinger operator and conditional Feynman-Kac gauge. J. Math. Anal. Appl. 116 (1986), 309-334.

[53] Q. S. Zhang. The boundary behavior of heat kernels of Dirichlet Laplacians. J. Diff. Eqs. 182 (2002), 416-430.

(Cho) Department of Mathematical Sciences, Seoul National University, Building 27, 1 Gwanak-ro, GWANAK-Gu Seoul 08826, Republic of Korea

Email address: soobin15@snu.ac.kr

(Kim) Department of Mathematical Sciences and Research Institute of Mathematics, Seoul National

University, Building 27, 1 Gwanak-ro, Gwanak-Gu Seoul 08826, Republic of Korea

Email address: pkim@snu.ac.kr

(Song) Department of Mathematics, University of Illinois, Urbana, IL 61801, USA

Email address: rsong@math.uiuc.edu

(Vondraček) Department of Mathematics, Faculty of Science, University of Zagreb, Zagreb, Croatia, Email address: vondra@math.hr 